\title{
HAUSDORFF DIMENSION AND CONFORMAL MEASURES OF FEIGENBAUM JULIA SETS
}

\author{
ARTUR AVILA AND MIKHAIL LYUBICH
}

\section{INTRODUCTION}

1.1. Statement of the results. One of the first questions usually asked about a fractal subset of $\mathbb{R}^{n}$ is whether it has the maximal possible Hausdorff dimension, $n$. It certainly happens if the set has positive Lebesgue measure. On the other hand, it is easy to construct fractal sets of zero measure but of dimension $n$. Moreover, this phenomenon is often observable for fractal sets produced by conformal dynamical systems, iterated rational functions or Kleinian groups. In particular, the analogy with Kleinian groups suggested that the Julia sets of Feigenbaum maps should have Hausdorff dimension two. In this paper we will show that this is not always the case.

A quadratic polynomial (or more generally, a quadratic-like map) is called Feigenbaum if it is infinitely renormalizable of bounded combinatorial type with a priori bounds (see $\S 2$ for a precise definition). Feigenbaum polynomials are remarkable dynamical systems whose geometry has been a focus of research for the past 25 years. By analogy with Kleinian groups, it was anticipated that their Julia sets have dimension 2, and this problem has been around for a while. However, in this paper we will show that this is not always the case:

Theorem A $(\mathrm{HD}<2)$. There exist Feigenbaum quadratic polynomials whose Julia sets $J(f)$ have Hausdorff dimension strictly less than 2.

Maps to which this theorem applies are infinitely renormalizable maps with "high" combinatorics. To the best of our knowledge, previous examples of Julia sets of Hausdorff dimension smaller than 2 were related to one of two mechanisms:

(1) definite expansion along the critical orbit,

(2) porosity (presence of definite holes in small scales) of the Julia set.

Note that both mechanisms fail for Feigenbaum maps.

Remark. We show in $[\mathrm{AL}]$ that in fact $\operatorname{HD}(J(f))$ can be arbitrarily close to 1 for some Feigenbaum Julia sets. Namely, when the combinatorics of the renormalization gets close to the Chebyshev one (of the map $z \mapsto z^{2}-2$ ), the dimension gets close to 1 .

It is still unknown whether there exist Feigenbaum Julia sets of positive area, or the ones with zero area but Hausdorff dimension two. Curiously, the affirmative answer to the former question would imply the affirmative answer to the latter one:

Received by the editors September 20, 2004.

2000 Mathematics Subject Classification. Primary 37F25; Secondary 37F35. 
Theorem B. Assume that a renormalization horseshoe $\mathcal{A}$ (see $\S 2.5)$ contains maps $g_{+}$and $g_{0}$ such that area $\left(J\left(g_{+}\right)\right)>0$ while area $\left(J\left(g_{0}\right)\right)=0$. Then it contains a map $f$ such that area $(J(f))=0$ but $\operatorname{HD}(J(f))=2$.

In the course of this paper, we give a criterion for area $J(f)>0$ which can provide an efficient numerical test on this property.

Along with the Hausdorff dimension, there are other natural geometric objects and quantities associated with Julia sets:

- The Poincaré series

$$
\Xi(\delta) \equiv \Xi_{\delta}(f, z)=\sum_{n=0}^{\infty} \sum_{f^{n} \zeta=z} \frac{1}{\left|D f^{n}(\zeta)\right|^{\delta}}, \quad z \in \mathbb{C} \backslash \mathcal{O},
$$

where $\mathcal{O}$ is the postcritical set of $f$.

- The critical exponent $\delta_{\mathrm{cr}}=\delta_{\mathrm{cr}}(f)$ which separates convergent exponents of the Poincaré series from the divergent ones.

- The hyperbolic dimension $\operatorname{HD}_{\text {hyp }}(J)$ of the Julia set $J=J(f)$, that is, the supremum of the dimensions of all invariant hyperbolic subsets of $J$.

- Conformal measures on the Julia sets, that is, the measures that transform according to the rule

$$
\mu(f X)=\int_{X}|D f|^{\delta} d \mu
$$

for any measurable set $X$ such that $f \mid X$ is injective. By Sullivan's Theorem [S3], the Julia set $J$ supports a conformal measure with exponent $\delta_{\mathrm{cr}}$.

- The minimum exponent $\delta_{*}=\delta_{*}(f)$ is the infimum of the exponents of all conformal measures on the Julia set.

The following general relation between the above quantities has been known:

$$
\delta_{*}=\operatorname{HD}_{\text {hyp }}(J) \leq \mathrm{HD}(J),
$$

where the equality is due to Denker-Urbański and Przytycki [DU, Pz1] while the inequality is trivial. If moreover the Julia set has zero area, then the inequality

$$
\mathrm{HD}(J) \leq \delta_{\mathrm{cr}}
$$

holds for a large class of maps (including Feigenbaum maps), a result due to Bishop ${ }^{1}$ [B] (dynamically interpreted in [GS]). However, it is not known whether these quantities in general coincide. In the case of Feigenbaum maps, (and in fact, for quite a general class of rational maps (see $\S 10)$ ), we can resolve this issue:

Theorem $\mathbf{C}$ (Critical exponents and dimensions). Let $f$ be a Feigenbaum map. Then

$$
\delta_{\mathrm{cr}}(f)=\delta_{*}(f)=\operatorname{HD}_{\text {hyp }}(J) .
$$

Moreover, if area $J(f)=0$, then

$$
\operatorname{HD}_{\text {hyp }}(J)=\operatorname{HD}(J) .
$$

It is quite surprising that the last assertion can be reversed in the case of periodic combinatorics:

\footnotetext{
${ }^{1}$ Actually Bishop has proved the a priori stronger result that (under the assumption that the Julia set has zero Lebesgue measure) $\delta_{\mathrm{cr}} \geq \overline{\mathrm{MD}}$, the upper Minkowski dimension. Together with this result of Bishop, our Theorem $\mathrm{C}$ below shows that the Minkowski dimension of a Feigenbaum Julia set is equal to its Hausdorff dimension.
} 
Theorem D (Positive area and hyperbolic dimension). Let $f$ be a Feigenbaum map with periodic combinatorics. If area $(J)>0$, then

$$
\operatorname{HD}_{\text {hyp }}(J)<\operatorname{HD}(J)=2 .
$$

At this moment we cannot tell whether this result is positive or negative (that is, whether it gives an interesting property of certain Julia sets or indicates that those Julia sets should not exist).

Putting Theorems C and D together we obtain the following amusing statement: A Feigenbaum map with periodic combinatorics has a Julia set of zero Lebesgue measure if and only if its hyperbolic and Hausdorff dimensions coincide.

It is known (Prado $[\mathrm{P}]$ ) that any conformal measure on a Feigenbaum Julia set is ergodic (and hence there exists at most one conformal measure for any given exponent $\delta$ ). Another traditional question asked about quasi-invariant measures is whether they are dissipative or conservative. ${ }^{2}$ Recall that an ergodic measure is called conservative if it satisfies the conclusion of the Poincaré Recurrence Theorem and is called dissipative otherwise.

Theorem E (Conformal measures). Let $f$ be a Feigenbaum map. Then for any $\delta \geq \delta_{*}(f)$, the Julia set $J(f)$ supports a unique conformal measure $\mu_{\delta}$ with exponent $\delta$. This measure is atomless. If $\mu_{\delta}$ is conservative, then $\delta=\delta_{*}<2$. Moreover, both dissipative and conservative alternatives with $\delta=\delta_{*}<2$ are realizable for some Feigenbaum quadratic-like maps.

This gives first examples of dissipative $\delta_{*}$-conformal measures with $\delta_{*}<2$.

Remark 1.1. Shishikura [Sh1] has constructed a large family of quadratic Julia sets with $\delta_{*}=2$. The corresponding 2-conformal measures are probably always dissipative, and our results show that this is the case at least when the critical orbit is not dense in the Julia set (see Corollary 3.2). To see that it can indeed happen, one can consider a renormalizable quadratic map whose renormalization belongs to Shishikura's examples.

1.2. Methods. In this paper we develop an efficient method to estimate the Poincaré series by comparing its values on different renormalization levels. For periodic points of renormalization this method leads to a Recursive Quadratic Estimate for the Poincaré series. Let $A^{n}$ be the fundamental annuli on consecutive renormalization levels. Then we show that the average Poincaré series,

$$
\omega \equiv \omega_{m}(\delta)=\frac{1}{\operatorname{area} A^{m}} \int_{A^{m}} \Xi_{\delta}(x) d x,
$$

satisfies an estimate $\omega \leq P_{\delta}(\omega)$, where $P_{\delta}$ is a quadratic polynomial (with positive coefficients) whose coefficients continuously depend on $\delta$ near 2 . It gives a bound of the average Poincaré series $\omega$ by a real fixed point of $P_{\delta}$ if it exists. Moreover, coefficients of $P_{2}$ involve areas of points that escape annuli $A^{m}$, and one can see that if substantial masses of points escape the $A^{m}$, then $P_{2}(x)$ has an attracting fixed point. Persistence of this point under a perturbation of $\delta$ yields convergence of the Poincaré series for $\delta$ slightly smaller than 2 , implying that $\delta_{\text {cr }}<2$.

More generally, this leads us to the following Trichotomy. For any Feigenbaum map with periodic combinatorics one of the following three possibilities occurs:

\footnotetext{
${ }^{2}$ For Kleinian groups this question is related to existence of the Green function on the associated 3-manifold (see $[\mathrm{Ah}]$ ).
} 
1) Lean case: substantial masses of points escape the annuli $A^{n}$ to the outer regions. In this case

$$
\operatorname{HD}(J)=\operatorname{HD}_{\text {hyp }}(J)<2 .
$$

2) Balanced case: there is a balance between masses of points escaping in and out the fundamental annuli. In this case

$$
\text { area } J=0 \text { but } \operatorname{HD}_{\text {hyp }}(J)=2 \text {. }
$$

3) Black hole case: the critical point acts like a black hole that attracts huge masses of matter, so that substantial masses escape the annuli $A^{n}$ to the inner regions. In this case

$$
\text { area } J>0 \text { but } \operatorname{HD}_{\text {hyp }}(J)<2 .
$$

In the case of Feigenbaum maps with high combinatorics, the critical point is lean, which yields Theorem A. Theorem D is incorporated into the black hole case. Heuristically, phase transition from the lean case to the black hole case can occur only through the balanced case, indicating that Theorem B should hold.

Theorem $\mathrm{C}$ is based upon a new construction of a $\delta$-conformal measure by taking a properly truncated Poincaré series $\Xi_{\delta}^{t r}(f, z)$, putting the corresponding measure on the preimages of $z$, and letting $z \rightarrow 0$. This allows us to demonstrate that conformal measures exist for any $\delta \geq \delta_{*}$, which is the key step of the proof (see also Theorem E).

Dissipativity of the $\delta$-conformal measure is equivalent to convergence of the Poincaré series $\Xi_{\delta}$. A simple argument shows that $\Xi_{\delta_{*}}(R f, z)$ is convergent, provided the dimension does not drop under the renormalization:

$$
\operatorname{HD}_{\text {hyp }}(J(R f))=\operatorname{HD}_{\text {hyp }}(J(f)) \equiv \delta_{*},
$$

which holds, e.g., for renormalization fixed points $f=R f$. On the other hand, if the dimension drops,

$$
\operatorname{HD}_{\text {hyp }}(J(R f))<\operatorname{HD}_{\text {hyp }}(J(f)),
$$

then we find a linear recursive estimate for the Poincaré series (this time averaging over the conformal measure $\mu_{\delta_{*}}$ on the annuli $A^{n}$ ), which would yield convergence of $\Xi_{\delta}$ for some $\delta<\delta_{*}$ if the measure $\mu_{\delta_{*}}$ were dissipative (contradicting that $\delta_{*}$ is the minimum exponent).

Finally, we give two proofs that the drop condition (1.5) is satisfied for some Feigenbaum maps, which completes the proof of Theorem E. One of them, based on methods of quasiconformal deformations, shows that

$$
\sup _{f \in \mathcal{H}} \operatorname{HD}_{\text {hyp }}(f)=2,
$$

where $\mathcal{H}$ is any hybrid class of Feigenbaum quadratic-like maps. Another one is based on a dynamical interpolation in an appropriate renormalization horseshoe.

Our methods also work in the generalized renormalization contexts, with Fibonacci maps as main examples. They can also be applied to the real dynamics, where it is known that "wild attractors" exist [BKNS] (real analogue of the phenomenon of "positive area Julia sets"), which yields a real life version of Theorems B and D.

Also, in the search for Feigenbaum Julia sets of positive area, it might be a good idea to consider maps of higher degree (that is, $\geq 2$ ) as well. Let us remark with this respect that all the results and proofs of this paper are still valid for 
(unicritical) Feigenbaum maps of arbitrary degree (with only terminological and notational adjustments).

1.3. Some history. Geometric measures and Hausdorff dimension of limit and Julia sets are popular themes in holomorphic dynamics.

First examples of a conformal dynamical system with the limit set of measure zero but Hausdorff dimension 2 appeared in the works of Thurston [Th] and Sullivan [S2] on Kleinian groups. On the other hand, the limit set of a geometrically finite Kleinian group ${ }^{3}$ has Hausdorff dimension less than 2 [Bo], [S4], [T]. In this context, the Hausdorff dimension problem was eventually resolved in full by Bishop and Jones [BJ]: the limit set $\Lambda(\Gamma)$ of a (finitely generated) Kleinian group has Hausdorff dimension 2 if and only if the group is geometrically infinite. Also, it has been recently announced that the limit set of any finitely generated Kleinian group has zero area, which settles a long-standing Ahlfors Conjecture; see [Ag, CG].

Another basic class of conformal dynamical systems is given by iterates of a single endomorphism, rational or transcendental. The exponential map $z \mapsto \lambda e^{z}, \lambda<e^{-1}$, was the first example of such an endomorphism whose Julia set has zero area [EL1] but Hausdorff dimension 2 [Mc1]. Examples of entire functions with Julia sets of positive area were given in [EL2, Mc1]. For other interesting properties of the measure and dimension of transcendental Julia sets, see [L2, R, Kar, SZ, UZ, MU].

The first examples of rational Julia sets with Hausdorff dimension two were constructed by Shishikura [Sh1] (see also [Mc5]). Indeed, Shishikura demonstrated that this phenomenon is generic for quadratic polynomials $z \mapsto z^{2}+c$ with $c$ on the boundary of the Mandelbrot set. All these Julia sets have zero area [L3, Sh2].

On the other hand, the works of McMullen [Mc4] (see also [Pe]) and Przytycki [Pz2] (see also [PR, GS]) showed that the situation in the iteration theory is more complicated than in the theory of Kleinian groups: There exist geometrically infinite Julia sets with Hausdorff dimension strictly less than two. Here a map is called geometrically finite if all the critical points on the Julia set are non-recurrent (see $[\mathrm{LM}])$. The Hausdorff dimension of any geometrically finite Julia set is strictly less than 2, provided it is not the whole sphere (see [ADU, CJY, U1, Mc5]).

Recently, Buff and Chéritat $[\mathrm{BC}]$ have shown the existence of quadratic polynomials with a Julia set of positive area (see also an alternative approach by Gaidashev and Yampolski [GY]). As we have already mentioned, it is still unknown whether there exists a Feigenbaum map whose Julia set has positive area. However, it is known that many Feigenbaum Julia sets have zero area [Y]. (It is essentially the same class of maps for which a priori bounds had been established in [L5] and to which our Theorem A applies.)

McMullen [Mc3] has suggested that in the dictionary between rational maps and Kleinian groups, Feigenbaum maps correspond to hyperbolic 3-manifolds that fiber over the circle. Using this analogy, he proved that the critical point lies "deep" (in some precise geometric sense) inside any Feigenbaum Julia set. This strongly suggested that these sets should have the maximal possible Hausdorff dimension. However, as we have already mentioned, these points can be lean in the measuretheoretic sense, which leads to the opposite conclusion.

For Fuchsian groups, conformal measures were introduced by Patterson $[\mathrm{Pa}]$; the construction was extended to the Kleinian case by Sullivan [S1]. See [N] for

\footnotetext{
${ }^{3}$ We follow the classical convention that the limit set of a Kleinian group is not the whole sphere.
} 
a discussion of their ergodic properties. Ergodic and conservativity/dissipativity properties of conformal measures on Julia sets were studied, among other papers, in [L1, ADU, Mc2, U2, P, Ba, BM, GS] (part of this work was motivated by [BL] concerning real dynamics).

For the relations between the critical exponent and Hausdorff dimension for Kleinian groups see [BJ] (compare Theorem C).

1.4. Organization of the paper. We begin the paper $(\S 2)$ with basic material concerning the Hausdorff dimension, Poincaré series, conformal measures, and renormalization of quadratic-like maps. In $\S 3$ we study scaling and ergodic properties of conformal measures, and prove Theorem $\mathrm{C}$ and part of Theorem E.

Section 4 is the central one in the paper: here we derive the recursive quadratic estimates. In $\S 5$ we deduce from them the trichotomy. It immediately yields Theorem D and provides us with the "leanness" condition for Theorem A.

In $\S 6$ and $\S 7$ we complete a proof of Theorem E. In $\S 7.2$ we study how the Hausdorff dimension $h(g) \equiv \operatorname{HD}(J(g))$ varies within hybrid classes of Feigenbaum maps. One of the consequences of our analysis is the following: If $f$ has stationary combinatorics, then the function $h(g)$ is either identically equal to 2 , or else $\operatorname{Im} h=$ $\left[h\left(f_{*}\right), 2\right)$ where $f_{*}$ is the associated renormalization fixed point.

In $\S 8$, we prove Theorem B.

In $\S 9$ we describe a class of well controlled Feigenbaum maps [L5] whose Julia sets are lean at the critical point [Y], which completes the proof of the first assertion of Theorem A. The description is given in terms of the principal nest of the Yoccoz puzzle.

$\S 10$ contains various remarks and some open problems.

The paper is concluded with two appendices. Appendix A is of a technical nature: here we construct fundamental domains for Feigenbaum maps satisfying nice Markov properties. In Appendix B we analyze conformal measures on towers which yields sharper scaling properties of conformal measures on the Julia sets.

\section{BACKGROUND}

2.1. Notations and terminology. $\mathbb{C}$ is the complex plane;

$\mathbb{N}=\{0,1, \ldots\}$ is the set of natural numbers;

$\mathbb{Z}$ is the set of integers;

$\mathbb{D}_{r}(z)=\{\zeta:|z-\zeta|<r\}, \mathbb{D}_{r} \equiv \mathbb{D}_{r}(0), \mathbb{D} \equiv \mathbb{D}_{1} ;$

$U \Subset V$ means that $U$ is compactly contained in $V$, i.e., $\operatorname{cl} U$ is compact and is contained in $V$.

A domain is a connected open set. By a (topological) disk we will mean a simply connected domain $U \subset \mathbb{C}$. A Jordan disk is a topological disk whose boundary is a Jordan curve. The filling of a domain $D$ is the union of all Jordan disks with boundary contained in $D$ : it is the smallest simply connected set containing $D$. Given a family of topological disks, we say that they have a bounded shape if they are quasi-disks with uniformly bounded dilatation. A (topological) annulus $A \subset \mathbb{C}$ is a doubly connected domain. Any topological annulus can be conformally uniformized by a round annulus $\{z: r<|z|<R\}$ (where possibly $r=0$ or $R=\infty$ ). The modulus of the topological annulus, $\bmod (A)$, is defined as $\log (R / r)$.

The Lebesgue measure ( $\equiv$ area) of a measurable set $X \subset \mathbb{C}$ will be denoted by area $X$ or $|X|$. If $X, Y \subset \mathbb{C}$ are measurable and $|Y|>0$, we let $p(X \mid Y)=$ $|X \cap Y| /|Y|$. 
The distortion of a (holomorphic) univalent map $f: U \rightarrow \mathbb{C}$ is defined as

$$
\sup _{z, \zeta \in U} \frac{|D f(z)|}{|D f(\zeta)|} .
$$

"Quasi-conformal" will be abbreviated as "qc".

For a natural $s \geq 2$, let $\Sigma_{s}$ denote the space of two-sided sequences $\left(\epsilon_{k}\right)_{k=-\infty}^{\infty}$ in $s$ symbols: $\epsilon_{k} \in\{1, \ldots, s\}$. The shift transformation $\sigma$ on this space is called a Bernoulli shift.

The forward orbit of a point $z$ under $f$ is denoted $\operatorname{as} \operatorname{orb}(z) \equiv \operatorname{orb}_{f} z=\left\{f^{n} z\right\}_{n=0}^{\infty}$; its limit set is denoted as $\omega(z)$.

$a \asymp b$ means that $C^{-1}<a / b<C$ with a constant $C>0$ independent of the particular $a$ and $b$ under consideration.

Assertion that "some property is satisfied for $\delta \approx a$ " naturally means that it is satisfied for all $\delta$ sufficiently close to $a$.

2.2. Hyperbolic sets. Let $X \subset \mathbb{C}$ be a compact set invariant under a certain holomorphic map $f$ defined in a neighborhood of $X$. It is called hyperbolic if there exist $C>0$ and $\lambda>1$ such that for all $z \in X$

$$
\left|D f^{n}(z)\right| \geq C \lambda^{n}, n=0,1, \ldots
$$

2.3. Quadratic-like maps. Let $\mathbb{U} \Subset \mathbb{V}$ be two topological disks. A (holomorphic) branched covering of degree $d f: \mathbb{U} \rightarrow \mathbb{V}$ is called a polynomial-like map of degree $d$. If $d=2$, the map is called quadratic-like.

The filled Julia set $K(f)$ of a polynomial-like map is defined as the set of nonescaping points (where "escaping" means landing at the fundamental annulus $\mathbb{V} \backslash \mathbb{U}$ under some iterate of $f$ ), and the Julia set $J(f)$ is defined as the boundary of $K(f)$. In the quadratic-like case, these sets are either Cantor or connected depending on whether the critical point itself is escaping or not.

Any quadratic-like map has two fixed points counted with multiplicity. In the case of a connected Julia set these two points can be dynamically distinguished. One of them, usually denoted by $\alpha$, is either non-repelling or dividing, i.e., removing of it makes the Julia set disconnected. Another one, denoted by $\beta$, is always nondividing.

If a quadratic-like map is considered up to choice of domains $\mathbb{U}$ and $\mathbb{V}$, then it should be more carefully called a quadratic-like germ. More precisely, one says that two quadratic-like maps with connected Julia sets represent the same germ if they have a common Julia set and coincide in a neighborhood of it. For such a germ, let $\bmod (f)=\sup \bmod (\mathbb{V} \backslash \mathbb{U})$, where the supremum is taken over all possible choices of domains $\mathbb{U}$ and $\mathbb{V}$. We will not make notational difference between maps and germs.

We will assume below that 0 is the critical point of $f$.

Two quadratic-like maps/germs $f$ and $g$ are called hybrid equivalent if there is a qc conjugacy $h$ between them with $\bar{\partial} h=0$ a.e. on the filled Julia set $K(f)$. By the Straightening Theorem [DH], any quadratic-like map $f$ with connected Julia set is hybrid equivalent to a unique quadratic polynomial $z \mapsto z^{2}+\chi(f)$, with $c=\chi(f)$ on the Mandelbrot set $M$. Let $\mathcal{H}_{f}$ stand for the hybrid class of $f$.

2.4. Feigenbaum maps. A quadratic-like germ $f: \mathbb{U} \rightarrow \mathbb{V}$ is called renormalizable if there exist $p=p(f)>1$ and topological disks $\mathbb{U}^{\prime} \Subset \mathbb{V}^{\prime}$ containing the critical point such that: 
- $g \equiv f^{p}: \mathbb{U}^{\prime} \rightarrow \mathbb{V}^{\prime}$ is a quadratic-like map with connected Julia set $J(g)$ called a pre-renormalization of $f$;

- the "little Julia sets" $f^{k} J(g), k=0,1, \ldots, p-1$, are pairwise disjoint except perhaps touching at their $\beta$-fixed points.

If the little Julia sets indeed touch, then one says that $f$ is immediately renormalizable (or that the renormalization is of satellite type). Otherwise the renormalization is called primitive.

A pre-renormalization considered up to affine conjugacy is called a renormalization of $f$. The renormalization of $f$ with minimal possible period is denoted $R f$.

The Mandelbrot set contains a plenty of canonical homeomorphic copies of itself. A quadratic-like map $f$ is renormalizable if and only if its straightening $\chi(f)$ belongs to one of these copies. The choice of this copy specifies the combinatorics of the renormalization.

Now one can inductively define an infinitely renormalizable map $f$; its successive renormalizations are denoted by $R^{m} f, m=0,1,2, \ldots$ If all these renormalizations have the same combinatorial type, one says that $f$ has a stationary type. If the sequence of the combinatorics is periodic, then $f$ has a periodic type. If the periods of all renormalizations are bounded, then $f$ has a bounded type.

One says that an infinitely renormalizable map has a priori bounds if

$$
\bmod \left(R^{n} f\right) \geq \epsilon>0, \quad n=0,1,2, \ldots
$$

Conjecturally a priori bounds are valid for all infinitely renormalizable maps of bounded type. So far, this conjecture has been confirmed for real maps (see [S5, $\mathrm{MS}]$ ) and complex maps with all renormalizations primitive [K].

An infinitely renormalizable quadratic-like map of bounded type with a priori bounds will also be called a Feigenbaum map.

2.5. Renormalization horseshoe. A renormalizable quadratic-like map is called the renormalization fixed (respectively, periodic) point if $R f=f$ (respectively, $R^{p} f=f$ for some $p$ ). More generally, let us pick a finite family $\mathcal{M}$ of little Mandelbrot copies $M_{k}, k=1, \ldots, s$. Assume that to any two-sided sequence $\bar{M}=$ $\left(M_{k(n)}\right)_{k=-\infty}^{\infty} \in \Sigma_{s}$ we can associate a quadratic-like germ $g_{\bar{M}}$ with uniform $a$ priori bounds which is renormalizable with combinatorics $M_{k(0)}$ and such that $R f_{\bar{M}}=f_{\sigma(\bar{M})}$ where $\sigma: \Sigma_{s} \rightarrow \Sigma_{s}$ is the Bernoulli shift. Then the family $\mathcal{A}_{\mathcal{M}}$ of these quadratic-like germs is called the renormalization horseshoe associated to the given family of copies. Notice that by definition, $\mathcal{A}$ is invariant under the renormalization, and $R: \mathcal{A}_{\mathcal{M}} \rightarrow \mathcal{A}_{\mathcal{M}}$ is topologically conjugate to the Bernoulli shift $\sigma$.

We say that a finite family $\mathcal{M}$ of little Mandelbrot copies is fine if for any one-sided sequence $\bar{M}_{+}=\left(M_{k(n)}\right)_{k=0}^{\infty}$ of these copies there is a quadratic-like germ which is renormalizable with combinatorics $\bar{M}_{+}$with a priori bounds. For instance, any family of real Mandelbrot copies is nice.

Theorem 2.1 ([S5, Mc3]). Any fine family $\mathcal{M}$ of Mandelbrot copies has an associated renormalization horseshoe $\mathcal{A}_{\mathcal{M}}$.

Notice that periodic points of renormalization are dense in the horseshoe $\mathcal{A}_{\mathcal{M}}$. 
Theorem 2.2 ([L8]). The horseshoe $\mathcal{A}_{\mathcal{M}}$ is hyperbolic with codimension-one stable foliation. Moreover, local stable manifolds of points $f \in \mathcal{A}_{\mathcal{M}}$ coincide with their hybrid classes.

2.6. Nest of little Julia sets. Let $f$ be a Feigenbaum quadratic-like map. Let us consider a sequence $f_{m}: \mathbb{U}^{m} \rightarrow \mathbb{V}^{m}$ of $m$-fold pre-renormalizations of $f$. Let $J \equiv J^{0} \supset J^{1} \supset J^{2} \supset \ldots$ stand for the corresponding nest of little Julia sets, $J^{m}=J\left(f_{m}\right)$. Then

$$
\mathcal{O} \equiv \mathcal{O}(f)=\omega(0)=\bigcap_{m \geq 0} \bigcup_{i \geq 0} f^{i}\left(J^{m}\right)
$$

is the postcritical set of $f$.

Lemma 2.3 (see, e.g., [Mc3]). There exist quadratic-like pre-renormalizations $f_{m}$ : $\mathbb{U}^{m} \rightarrow \mathbb{V}^{m}$ with the following properties:

(P1) $\operatorname{cl}\left(\mathbb{V}^{m} \backslash \mathbb{U}^{m}\right) \cap \mathcal{O}=\emptyset$;

(P2) unbranched a priori bounds: $\bmod \left(\mathbb{V}^{m} \backslash \overline{\mathbb{U}}^{m}\right) \geq \mu>0$ and $\bmod \left(\mathbb{U}^{m} \backslash J^{m}\right) \geq$ $\mu>0$

(P3) the disks $\mathbb{U}^{m}$ and $\mathbb{V}^{m}$ are quasidisks with bounded dilatation;

(P4) $\operatorname{diam} J^{m} \asymp \operatorname{diam} \mathbb{V}^{m}$.

All the constants depend only on the combinatorial and a priori bounds for $f$.

If $f$ is a renormalization fixed point, then there exists a dilation $\lambda>1$ such that $f_{m+1}=\lambda f_{m}\left(\lambda^{-1} z\right)$, and the above picture becomes scaling invariant: $\mathbb{V}^{m}=\lambda^{m} \mathbb{V}^{0}$, $\mathbb{U}^{m}=\lambda^{m} \mathbb{U}^{0}$, and $J^{m}=\lambda^{m} J^{0}$. In the case of renormalization periodic point, the scaling invariance holds on the subsequences of levels congruent modulo the period.

In what follows, the nests of domains $\mathbb{V}^{m}$ and $\mathbb{U}^{m}$ will always be assumed to satisfy properties (P1)-(P4). In the case of periodic combinatorics, they will be additionally assumed to be scaling invariant on the appropriates subsequences of levels.

2.7. Nice fundamental domains. Let us consider a Feigenbaum map $f: \mathbb{U} \rightarrow \mathbb{V}$ with pre-renormalizations $f_{n}: \mathbb{U}^{n} \rightarrow \mathbb{V}^{n}$. Along with the domains $\mathbb{V}^{n}$ and $\mathbb{U}^{n}$, we will also need simply connected domains $V^{n}$ and $U^{n}$ satisfying the following "nice" topological and geometric properties:

(C1) $\mathbb{V}^{n} \cap \mathcal{O}(f) \subset V^{n} \subset \mathbb{U}^{n}$,

(C2) $V^{n+1} \subset U^{n} \equiv f_{n}^{-1}\left(V^{n}\right)$,

(C3) $f^{k}\left(\partial V^{n}\right) \cap V^{n}=\emptyset, k \geq 0$ (compare with "nice intervals" of Martens [M1]),

(G1) $A^{n} \equiv V^{n} \backslash U^{n}$ is far from the postcritical set $\mathcal{O}(f)$ : it has bounded hyperbolic diameter in $\mathbb{V}^{n} \backslash \mathcal{O}(f)$,

(G2) $\operatorname{area}\left(A^{n}\right) \asymp \operatorname{area}\left(U^{n}\right) \asymp\left(\operatorname{diam}\left(U^{n}\right)\right)^{2} \asymp\left(\operatorname{diam}\left(V^{n}\right)\right)^{2}$.

The bounds (G1)-(G2) together with the unbranched a priori bounds will be called geometric bounds. By combinatorial bounds we will mean a bound on the renormalization period of all renormalizations of $f$.

In Appendix A we will construct such a nest of nice fundamental domains for any Feigenbaum map.

Remark 2.1. Condition (C3) implies that the first landing map to $V^{n}$ is particularly simple: its restriction to each component of its domain is a univalent map onto $V^{n}$. Due to (P1) each univalent pullback of $V^{n}$ extends to a univalent pullback of 
$\mathbb{V}^{n}$, so by the Koebe Distortion Lemma the first landing map to $V^{n}$ has bounded distortion.

Another consequence of $(\mathrm{C} 3)$ is the structure of the first return map to $V^{n}$. We have that $U^{n} \subset V^{n}$ and that $f_{n}: U^{n} \rightarrow V^{n}$ is a double covering. Thus $U^{n}$ is one of the components of the domain of the first return map to $V^{n}$. All other components are univalent pullbacks of $V^{n}$.

2.8. Hausdorff dimension. Given a $\delta \geq 0$, the Hausdorff $\delta$-measure $h_{\delta}$ is defined as follows:

$$
h_{\delta}(X)=\lim _{\epsilon \rightarrow 0} \inf \sum\left(\operatorname{diam} U_{i}\right)^{\delta},
$$

where the infimum is taken over all coverings of $X$ by sets $U_{i}$ of diameter at most $\epsilon>$ 0 . For any $X$ there is a unique critical exponent separating infinite and vanishing values of the measure $h_{\delta}$. This exponent is called the Hausdorff dimension of $X$ and is denoted by $\operatorname{HD}(X)$.

Given a Borel measure $\mu$, the Hausdorff dimension $\operatorname{HD}(\mu)$ is defined as the infimum of the $\mathrm{HD}(X)$ as $X$ runs over all measurable sets of full measure. Local dimension $\operatorname{HD}_{\mu}(z)$ of a measure $\mu$ at a point $z$ is defined as

$$
\lim _{r \rightarrow 0} \frac{\log \mu\left(\mathbb{D}_{r}(z)\right)}{\log r}
$$

provided this limit exists. If the limit does not exist, one can still consider upper and lower local dimensions, $\overline{\mathrm{HD}}_{\mu}(z)$ and $\underline{\mathrm{HD}}_{\mu}(z)$, by taking upper and lower limits in $(2.1)$.

The hyperbolic dimension $\operatorname{HD}_{\text {hyp }}(X)$ of an invariant set $X$ is defined as the supremum of the dimensions of all invariant hyperbolic subsets of $X$.

Theorem 2.4 ([Z]). For any polynomial-like map $f: U \rightarrow V$ with a connected Julia set, there is an alternative:

- Either $J(f)$ is a real analytic curve;

- or $\operatorname{HD}(J(f))>1$.

\subsection{Conformal measures and critical exponents. The Poincaré series}

$$
\Xi_{\delta}(z) \equiv \Xi_{\delta}(f, z), \quad z \in \mathbb{C} \backslash \mathcal{O},
$$

was defined in (1.1) in the Introduction. Note that by the Koebe Distortion Lemma it has a bounded oscillation on any compact set $K \subset \mathbb{V} \backslash \mathcal{O}$ :

$$
\Xi_{\delta}(f, z) \leq C \Xi_{\delta}(f, \zeta), \quad z, \zeta \in K,
$$

where the constant $C$ depends only on the hyperbolic diameter of $K$ in $\mathbb{V} \backslash \mathcal{O}$,

By definition, the critical exponent $\delta_{c r}=\delta_{c r}(f)$ separates convergent values of $\delta$ in $\Xi_{\delta}$ from the divergent ones: the Poincaré series is convergent for $\delta>\delta_{\text {cr }}$ and divergent for $\delta<\delta_{\mathrm{cr}}$. Notice that by $(2.2), \delta_{\mathrm{cr}}$ is independent of the particular choice of $z \in \mathbb{V} \backslash \mathcal{O}$. For $\delta=\delta_{\text {cr }}$ the Poincaré series can behave in both ways, and $f$ is called of convergent or divergent type depending on it.

Taking a wandering disk $D \Subset \mathbb{V} \backslash J$ (i.e., $f^{-n} D \cap f^{-m} D=\emptyset$ for $m \neq n$ ), we see that

$$
\Xi_{2}(z) \asymp \bigcup_{n=0}^{\infty} \operatorname{area}\left(f^{-n}(D)\right)<\infty,
$$

so that $\delta_{\text {cr }} \leq 2$. One can also show that $\delta_{\text {cr }}>0$. In fact, we have:

Lemma 2.5. If the Julia set $J(f)$ is connected, then $\Xi_{1}=\infty$, so that $\delta_{\mathrm{cr}} \geq 1$. 
Proof. Let $\Gamma_{n}=\partial\left(f^{-n} \mathbb{U}\right)$. Then $\Xi_{1} \asymp \sum \operatorname{length}\left(\Gamma_{n}\right)=\infty$.

A quasi-invariant measure $\mu$ is called $\delta$-conformal if its Radon-Nikodym Jacobian $d\left(f^{*} \mu\right) / d \mu$ is equal to $|D f|^{\delta}$, which is equivalent to the transformation rule (1.2). This transformation rule and density of preimages on the Julia set imply that $\operatorname{supp}(\mu)=J(f)$ for any conformal measure $\mu$ on the Julia set.

Theorem 2.6 (Sullivan [S3]). Any polynomial-like map $f$ has at least one $\delta_{\mathrm{cr}^{-}}$ conformal measure $\mu$ on its Julia set.

We have also defined in the Introduction the minimal exponent $\delta_{*}=\delta_{*}(f)$, the infimum of the exponents $\delta$ for which $f$ admits a $\delta$-conformal measure on the Julia set.

Theorem 2.7 (Denker-Urbański and Przytycki [DU, Pz1]). For any polynomiallike map,

$$
\delta_{*}(f)=\operatorname{HD}_{\text {hyp }}(J(f)) .
$$

Moreover, $f$ admits a $\delta_{*}$-conformal measure on its Julia set.

Let us also mention the following simple property:

Lemma 2.8. The exponent $\delta_{*}(f)=\operatorname{HD}_{\text {hyp }}(f)$ depends lower semicontinuously on the quadratic-like map $f$.

Recall that an $f$-quasi-invariant measure $\mu$ on $J$ is called ergodic if there is no decomposition $J=X_{1} \cup X_{2}$ into two invariant measurable subsets of positive measure.

Theorem 2.9 (Prado $[\mathrm{P}]$ ). Let $f$ be a Feigenbaum map. Then any conformal measure $\mu$ on $J(f)$ is ergodic. Hence for any given exponent $\delta$, there exists at most one $\delta$-conformal measure.

2.10. Hyperbolic repellers. For a Feigenbaum map $f$, there is an easy way to contruct hyperbolic sets. Namely, for $r>0$, let

$$
J^{r}=\left\{z:\left|f^{n} z\right| \geq r, n=0,1, \ldots\right\} .
$$

It is a forward-invariant compact set that does not contain the critical point. Since the postcritical set $\mathcal{O}$ is minimal, it does not intersect $\mathcal{O}$ either. This implies hyperbolicity in a standard way (e.g., by using the hyperbolic metric in $\mathbb{C} \backslash \mathcal{O}$ ).

However, to develop a good theory, one needs to impose some extra assumptions on a hyperbolic set.

Given a holomorphic map $f: U \rightarrow \mathbb{C}$, a (compact invariant) hyperbolic set $Q \subset U$ is called a hyperbolic repeller if the restriction $f: Q \rightarrow Q$ is an open map. The theory of hyperbolic repellers is well developed (see [PU]), in particular, it is known that $\operatorname{HD}(Q)=\delta_{\text {cr }}(f \mid Q)$.

A closed Jordan disk $D$ is called a puzzle piece if $f^{k}(\partial D) \cap$ int $D=\emptyset$ for every $k \geq 0$ and $\partial D$ intersects the Julia set $J$ in finitely many periodic or pre-periodic points. Let us say that a puzzle piece $D$ is suitable if there exists $n=n(D)>0$ and a relative neighborhood $V$ of $\partial D \cap J$ in $D \cap J$ such that $f^{n}(V) \subset D$.

Lemma 2.10 (compare $[\mathrm{J}]$ ). For any Feigenbaum map, there is a sequence of suitable critical puzzle pieces shrinking to 0 . 
Proof. Let $f_{m}$ be the $m$ th pre-renormalization of $f$ with period $p_{m}$. Let $\beta_{m}$ be its $\beta$-fixed point, and let $\mathcal{R}_{m}$ be the union of external rays (of $f$ ) landing at $\beta_{m}$. Let $V_{m}$ be the closure of the component of $\mathbb{C} \backslash\left(\mathcal{R}_{m} \cup\left(-\mathcal{R}_{m}\right)\right)$ containing 0 (see, e.g., $[\mathrm{L} 5, \S 2.5]$ for more details). Then the sets

$$
V_{m}^{l}=\left\{z: f^{p_{m} k}(z) \in V, k=0,1, \ldots, l\right\}
$$

are suitable puzzle pieces shrinking to the filled Julia set $K\left(f_{m}\right)$ as $l \rightarrow \infty$. Since the Julia sets $K\left(f_{m}\right)$ shrink to 0 , the conclusion follows.

This implies the following useful statement:

Lemma 2.11. Any hyperbolic set of a Feigenbaum map $f$ can be enlarged to a hyperbolic repeller.

Proof. By Lemma 2.10, for any hyperbolic set $X$, there is a suitable puzzle piece $D$ containing the critical point which does not intersect $X$. Let $Y=J \backslash \operatorname{int} D$, and let $Q=\bigcap_{n \in \mathbb{N}} f^{-n}(Y)$ be the maximal invariant set containing $X$. Then $Q$ is a hyperbolic repeller containing $X$.

2.11. Poincaré series in the hyperbolic case. Let us collect here some basic estimates on Poincaré series. They show that in the hyperbolic case the Poincaré series cannot blow up: it depends continuously on both $\delta$ and $f$.

Let $\Delta$ be the set of all continuous maps $f$ defined over a compact set $X \subset \mathbb{C}$ that admit a holomorphic extension to a neighborhood of $X$. We say that $f_{n}: X_{n} \rightarrow \mathbb{C}$ converge to $f: X \rightarrow \mathbb{C}$ is $X_{n} \rightarrow X$ in the Hausdorff topology and there exists a neighborhood $U$ of $X$ such that for every $n$ sufficiently big, $f_{n}$ admits a holomorphic extension to $U$ and the $f_{n}: U \rightarrow \mathbb{C}$ converge to $f: U \rightarrow \mathbb{C}$ uniformly. We will assume that $X$ has no isolated points (so that derivatives of a function $f: X \rightarrow \mathbb{C}$ of class $\Delta$ are well defined).

Lemma 2.12. Let $f: X \rightarrow \mathbb{C}$ be a map of class $\Delta$ without critical points. If $\delta>0$, $K>0$ are such that

$$
\Xi_{\delta}(f, x)<K
$$

for every $x \in X$, then for every $\tilde{K}>K$, there exists $\tilde{\delta}<\delta$ such that if $\tilde{f}: \tilde{X} \rightarrow \mathbb{C}$ is close to $f: X \rightarrow \mathbb{C}$, then for every $x \in \tilde{X}$, we have $\Xi_{\tilde{\delta}}(\tilde{f}, x)<\tilde{K}$.

Proof. For every $x \in X$, let $N_{x} \geq 0$ be minimal such that

$$
\sum_{f^{N_{x}}(y)=x}\left|D f^{N_{x}}(y)\right|^{-\delta}<\frac{1}{2 K} .
$$

Notice that $N_{x}$ is upper semicontinuous, so $N=\sup N_{x}<\infty$. It follows that

$$
\sum_{f^{N}(y)=x}\left|D f^{N}(y)\right|^{-\delta}=\sum_{f^{N_{x}} z=x}\left|D f^{N_{x}}(z)\right|^{-\delta} \sum_{f^{N-N_{x}}(y)=z}\left|D f^{N-N_{x}}(y)\right|^{-\delta}<\frac{1}{2}
$$


Choose an integer $M>0$ such that $2^{M-1}>K /(\tilde{K}-K)$. If $\tilde{f}: \tilde{X} \rightarrow \mathbb{C}$ is close to $f: X \rightarrow \mathbb{C}$ and $\tilde{\delta}$ is close to $\delta$, we still have, for every $x \in \tilde{X}$,

$$
\begin{gathered}
\sum_{\tilde{f}^{N}(y)=x}\left|D \tilde{f}^{N}(y)\right|^{-\tilde{\delta}}<\frac{1}{2}, \\
\sum_{m=0}^{M N-1} \sum_{\tilde{f}^{m}(y)=x}\left|D \tilde{f}^{m}(y)\right|^{-\tilde{\delta}}<K .
\end{gathered}
$$

Hence

$$
\begin{aligned}
\Xi_{\tilde{\delta}}(\tilde{f}, x)= & \sum_{m=0}^{M N-1} \sum_{\tilde{f}^{m}(y)=x}\left|D \tilde{f}^{m}(y)\right|^{-\tilde{\delta}} \\
& +\sum_{m=0}^{N-1} \sum_{\tilde{f}^{m}(z)=x}\left(\left|D \tilde{f}^{m}(z)\right|^{-\tilde{\delta}} \sum_{k=M}^{\infty} \sum_{\tilde{f}^{k N}(y)=z}\left|D \tilde{f}^{k N}(y)\right|^{-\tilde{\delta}}\right) \\
< & K+\sum_{k=M}^{\infty} K 2^{-k}<\tilde{K} .
\end{aligned}
$$

Lemma 2.13. Let $f: X \rightarrow \mathbb{C}$ be a map of class $\Delta$ without critical points. If the maximal invariant set $Q=\bigcap_{n \geq 0} f^{-n}(X)$ is hyperbolic, then there exists $K>0$ such that for every $x \in X$, we have $\Xi_{2}(f, x)<K$.

Proof. Let $\epsilon>0$ be such that $f$ admits a holomorphic extension to an $\epsilon$-neighborhood $U$ of $X$ and that $f \mid U$ has no critical points.

Let $Q_{m}=\bigcap_{0 \leq j \leq m} f^{-j}(X)$ so that $Q=\bigcap Q_{m}$. Hyperbolicity of $\bigcap_{m \geq 0} f^{-m}(X)$ implies that there exists $\rho>0$ such that if $y \in Q_{m}$, then there exists a Jordan domain $D_{y}^{m} \subset U$ such that $f^{m}: D_{y}^{m} \rightarrow \mathbb{D}_{\rho}\left(f^{m}(y)\right)$ is univalent and the diameter of $f^{k}\left(D_{y}^{m}\right), 0 \leq k \leq m$, is exponentially small in $m-k$.

It is easy to see that $Q$ has empty interior (otherwise $\left\{f^{m} \mid \operatorname{int} Q\right\}$ would form a normal family, contradicting the hyperbolicity of $f \mid Q)$. For $x \in X$, let us fix a compact set $Z_{x} \subset \mathbb{D}_{\rho}(x) \backslash Q$ of positive Lebesgue measure. We may assume that $\left\{Z_{x}\right\}_{x \in X}$ form a finite family. If $y \in Q_{m}$, let $Z_{y}^{m}=\left(f^{m} \mid D_{y}^{m}\right)^{-1}\left(Z_{f^{m}(y)}\right)$. Since the diameters of the $\{y\} \cup Z_{y}^{m}$ go to 0 as $m$ grows, there exists $M>0$ such that $Z_{y}^{m} \cap Z_{x}=\emptyset$ whenever $y \in Q_{m}, x \in X$, and $m \geq M$. It follows that $Z_{y}^{m} \cap Z_{y^{\prime}}^{m^{\prime}}=\emptyset$ if $\left|m^{\prime}-m\right| \geq M$. We also have $Z_{y}^{m} \cap Z_{y^{\prime}}^{m}=\emptyset$ whenever $f^{m}(y)=f^{m}\left(y^{\prime}\right)$ and $y \neq y^{\prime}$.

Notice that, by the Koebe Distortion Lemma, $\left|D f^{m}(y)\right|^{-2} \leq C\left|Z_{y}^{m}\right| /\left|Z_{f^{m}(y)}\right|$ for some $C>0$. Thus, for every $x \in X$,

$$
\sum_{m \geq 0} \sum_{\substack{f^{m}(y)=x, y \in Q_{m}}}\left|D f^{m}(y)\right|^{-2} \leq C \sum_{m \geq 0} \sum_{\substack{f^{m}(y)=x, y \in Q_{m}}} \frac{\left|Z_{y}^{m}\right|}{\left|Z_{x}\right|} \leq C M \frac{|U|}{\left|Z_{x}\right|} .
$$

Corollary 2.14. Let $f: X \rightarrow \mathbb{C}$ be a map of class $\Delta$ without critical points. If the maximal invariant set $Q=\bigcap_{n \geq 0} f^{-n}(X)$ is hyperbolic, then there exists $\delta<2$, $K>0$ such that for every $x \in X$ we have $\Xi_{\delta}(f, x)<K$. Moreover, $\delta$ and $K$ can be chosen uniform over a compact family of maps as above. 
3. Critical exponent, hyperbolic dimension, And Dissipativity

In what follows, $f$ is assumed to be a Feigenbaum map, unless otherwise is explicitly stated.

3.1. Dissipativity vs. conservativity. Recall that a measure $\mu$ is called dissipative if there exists a wandering set $X$ of positive measure, i.e., $\mu(X)>0$ and $f^{-n} X \cap f^{-m} X=\emptyset$ for all $m>n \geq 0$.

Lemma 3.1. Let $\mu$ be a $\delta$-conformal measure on $J$. Then the following properties are equivalent:

(i) $\mu$ is dissipative;

(ii) the Poincaré series $\Xi_{\delta}(z), z \in \mathbb{V} \backslash \mathcal{O}$, is convergent;

(iii) $f^{n} z \rightarrow \mathcal{O}$ for almost all $z$;

(iv) for any little Julia set $J^{m}$, almost any $\operatorname{orb}(z)$ is eventually absorbed by the cycle of little Julia sets, $\bigcup_{k=0}^{p-1} f^{k}\left(J^{m}\right)$ (here $p$ is the period of $J^{m}$ );

(v) $\mu\left(J^{m}\right)>0$ for all $m \in \mathbb{N}$;

(vi) $\mu\left(J^{1}\right)>0$.

If $\mu$ is conservative, then almost all orbits are dense in $J(f){ }^{4}$

Proof. (i) $\Longrightarrow$ (ii). Let $X$ be a wandering set of positive measure. Taking an appropriate local branch of $f^{-n}$ on $X$, we can easily construct a wandering set $X^{\prime} \subset \mathbb{D}(z, r)$ such that $\mathbb{D}(z, 2 r) \cap \mathcal{O}=\emptyset$. Then by the Koebe Distortion Lemma,

$$
\Xi_{\delta}(z) \asymp \sum \mu\left(f^{-n}\left(X^{\prime}\right)\right)<\infty .
$$

(ii) $\Longrightarrow$ (iii). Take any disk $D=\mathbb{D}(z, r)$ such that $\mathbb{D}(z, 2 r) \cap \mathcal{O}=\emptyset$. Then

$$
\sum \mu\left(f^{-n}(D)\right) \asymp \Xi_{\delta}(z)<\infty .
$$

By the Borel-Cantelli Lemma, for a.e. $\zeta \in J$, the orb $(\zeta)$ visits $D$ only finitely many times, which implies (iii).

(iii) $\Longrightarrow$ (iv). For a pre-renormalization $f^{p}: \mathbb{U}^{m} \rightarrow \mathbb{V}^{m}$ with period $p$, let $\mathbb{U}_{k}^{m}=f^{k}\left(\mathbb{U}^{m}\right), k=0,1, \ldots, p$ (so that $\mathbb{U}_{0}^{m}=\mathbb{U}^{m}$ and $\mathbb{U}_{p}^{m}=\mathbb{V}^{m}$ ).

Since the little Julia sets $f^{k} J^{m}$ may touch each other only at their $\beta$-fixed points, which are not contained in the postcritical set $\mathcal{O}$, there is a choice of prerenormalization such that the sets $\mathbb{U}_{1}^{m} \cap \mathcal{O}, \ldots \mathbb{U}_{p}^{m} \cap \mathcal{O}$ are pairwise disjoint. Then there exist neighborhoods $\Omega_{k}=f^{k}\left(\Omega_{0}\right) \subset \mathbb{U}_{k}^{m}$ of $\mathcal{O} \cap \mathbb{U}_{k}^{m}$ such that each $\Omega_{k}$ is disjoint from all $\Omega_{i}$ with $i \not \equiv k \bmod p$.

If $f^{n} z \rightarrow \mathcal{O}$, then

$$
f^{n} z \in \bigcup_{k=0}^{p-1} \Omega_{k}, \quad n \geq N .
$$

Let us take some moment $n \geq N$ for which $f^{n} z \in \Omega_{0}$. Then $f^{n+p} z \in \Omega_{p}$ which is disjoint from all $\Omega_{k}, k=1, \ldots, p-1$. Hence $f^{n+p}(z) \in \Omega_{0}$. It follows that $f^{n+p l} \in \Omega_{0} \subset \mathbb{U}^{m}$ for all $l=0,1, \ldots$ Thus $f^{n} z \in J^{m}$.

(iv) $\Longrightarrow(\mathrm{v})$. Take a little Julia set $J^{m}$. By (iv), $\bigcup_{n=0}^{\infty} f^{-n}\left(J^{m}\right)$ has full measure. This implies (v) by the $\delta$-covariance of $\mu$.

Of course, $(\mathrm{v}) \Longrightarrow$ (vi).

(vi) $\Longrightarrow$ (i). The full preimage $f^{-1}\left(J^{1}\right)$ consists of two symmetric components, $f^{p-1}\left(J^{1}\right)$ and $X$. If $\mu\left(J^{1}\right)>0$, then $X$ is a wandering set of positive measure.

\footnotetext{
${ }^{4}$ This last assertion holds for any rational map.
} 
Let us prove the last assertion. If it is not valid, then there is a disk $D$ (intersecting $J$ ) and a forward invariant set $X \subset J$ of positive measure such that $D \cap X=\emptyset$. Since $f^{N} D=J$ for some $N, D$ contains a set $Y$ of positive measure such that $f^{N} Y \subset X$. Hence $f^{n} Y \cap Y=\emptyset$ for $n \geq N$. This easily implies that

$$
\mu\left\{y \in Y: f^{n} y \notin Y, n=1,2, \ldots\right\}>0,
$$

which gives us a wandering set of positive measure.

Since $\Xi_{2}(z)<\infty$, we conclude:

Corollary 3.2 (compare [L1]). The 2-conformal measure $\delta_{2}$ is always dissipative. ${ }^{5}$

\subsection{No atoms.}

Proposition 3.3. For a Feigenbaum map, any conformal measure $\mu$ does not have atoms.

Proof. If $\mu$ has an atom $\mu(z) \delta_{z}$, then by $\delta$-covariance, all the preimages $\zeta \in f^{-n}(z)$ are also atoms with weight $\left|D f^{n}(\zeta)\right|^{-\delta}$. Clearly $z$ cannot be a (necessarily repelling) periodic orbit, so this implies that $\Xi_{\delta}(z)<\infty$. It follows from Lemma 3.1 that for any $m \in \mathbb{N}$, the $\operatorname{orb}(z)$ eventually lands on the little Julia set $J^{m}$. Let $n=n(m)$ be the first landing moment.

By the standard distortion estimates (coming from a priori bounds), there is a "little Julia set" $Q \ni z$ homeomorphically and with bounded distortion mapped under $f^{n}$ onto $J^{m}$.

Let $w=w_{m} \in J^{m}$ be a preimage of $f^{n} z$ under the pre-renormalization $f_{m}$, and let $\zeta=\zeta_{m} \in Q$ be the preimage of $w$ under $f^{n}$. By a priori bounds, $\left|D f_{m}(w)\right| \leq C$, and by the bounded distortion property,

$$
\frac{\left|D f^{n}(\zeta)\right|}{\left|D f^{n}(z)\right|} \leq K
$$

By the $\delta$-covariance,

$$
\mu(\zeta)=\frac{\mu(z)}{\left|D f_{m}(w)\right|^{\delta}}\left|\frac{D f^{m}(z)}{D f^{m}(\zeta)}\right|^{\delta} \geq(K C)^{-\delta} \mu(z) .
$$

Summing over all points $\zeta_{m}$ (and there are infinitely many of them), we accumulate an infinite mass - contradiction.

3.3. Minimal conformal measure. Let $\operatorname{Spec}(f)$ denote the conformal spectrum of $f$, that is, the set of exponents $\delta$ for which there exists a $\delta$-conformal measure on $J(f)$. Since weak* limits of conformal measures are conformal, we have:

Lemma 3.4. For any quadratic-like map $f,{ }^{6} \mathrm{Spec}(f)$ is a closed non-empty subset of $\mathbb{R}_{+}$.

In particular, the minimal exponent

$$
\delta_{*}=\delta_{*}(f)=\inf \operatorname{Spec}(f)
$$

belongs to the conformal spectrum. The corresponding $\delta_{*}$ conformal measure $\mu_{*}$ will be called minimal.

\footnotetext{
${ }^{5} \mathrm{By}$ analyzing the argument, the reader can check that this result is valid for any rational map whose postcritical set is not dense in the Julia set.

${ }^{6}$ The lemma holds for rational maps $f$ as well.
} 
All existing conformal measures, with the possible exception of the minimal one (in case $\delta_{*}<2$ ), are bound to be dissipative:

Lemma 3.5. Any conformal measure $\mu_{\delta}$ on $J$ is dissipative for $\delta>\delta_{*} \cdot{ }^{7}$

Proof. Assume $\mu_{\delta}$ is conservative. Then by the last assertion of Lemma 3.1, for $\mu_{\delta^{-}}$ a.e. $z$, there exists a sequence of moments $n_{k} \rightarrow \infty \operatorname{such}$ that $\operatorname{dist}\left(f^{n_{k}} z, \mathcal{O}\right)>2 r$, where $r>0$ is independent of $z$. Let $D_{k}(z)$ be the component of $f^{-n_{k}}\left(\mathbb{D}_{r}\left(f^{n_{k}} z\right)\right)$ containing $z$. Then by the Koebe Distortion Lemma, $D_{k}(z)$ is an oval of bounded shape such that the map $f^{n_{k}}: D_{k} \rightarrow \mathbb{D}_{r}\left(f^{n_{k}} z\right)$ has a bounded distortion (with an absolute bound). By covariance of conformal measures,

$$
\mu_{\delta}\left(D_{k}\right) \asymp\left(\operatorname{diam} D_{k}\right)^{\delta} \leq \epsilon_{k}\left(\operatorname{diam} D_{k}\right)^{\delta_{*}} \asymp \epsilon_{k} \mu_{\delta_{*}}\left(D_{k}\right),
$$

where $\epsilon_{k} \rightarrow 0$ as $k \rightarrow \infty$.

By the Besikovich Covering Lemma (see [Mat, §2.7]), there is a covering of a set $X$ of full $\mu_{\delta}$-measure with a family of balls $D_{k}$ with intersection multiplicity bounded by some absolute constant $N$. Hence

$$
\mu_{\delta}(J)=\mu_{\delta}(X) \leq \sum \mu_{\delta}\left(D_{k}\right) \leq \epsilon_{k} \sum \mu_{\delta^{*}}\left(D_{k}\right) \leq N \epsilon_{k} \mu_{\delta_{*}}(J) .
$$

Letting $k \rightarrow \infty$ we conclude that $\mu_{\delta}(J)=0$, a contradiction.

As the minimal measure $\mu_{*}$ is concerned, both dissipative and conservative options are realizable, at least for quadratic-like maps. In the following statement we give a condition for dissipativity; later on $(\S 6)$ we will deal with the conservative case.

Proposition 3.6. Assume $\delta_{*}(f)=\delta_{*}(R f) \equiv \delta_{*}$. Then the $\delta_{*}$-conformal measure of $R f$ is dissipative (and thus all conformal measures of $R f$ are dissipative).

Proof. Let $g: \mathbb{U}^{\prime} \rightarrow \mathbb{V}^{\prime}$ be a pre-renormalization of $f$. We let $\Xi_{*} \equiv \Xi_{\delta_{*}}$, and let $\mu_{*}$ and $\nu_{*}$ be $\delta_{*}$-conformal measures for $f$ and $g$, respectively. We will use the measure $\mu_{*}$ to prove dissipativity of $\nu_{*}$.

Take some disk $D=\mathbb{D}_{r}(z) \subset \mathbb{V}^{\prime} \backslash \mathbb{U}^{\prime}$ that intersects $J(f)$. Since $D$ is wandering under the dynamics of $g$,

$$
\Xi_{*}(g, z) \asymp \sum \mu_{*}\left(g^{-n}(D)\right)<\infty .
$$

By Lemma 3.1, $\nu_{*}$ is dissipative.

Lemma 3.7. Let $f$ be a Feigenbaum map which is recurrent under the dynamics of the renormalization operator (for instance, a renormalization fixed point). Then the $\delta_{*}$-conformal measure of $f$ is dissipative.

Proof. Note that Theorem 2.7 implies that $\delta_{*}$ is non-increasing under renormalization: $\delta_{*}(R f) \leq \delta_{*}(f)$ (since it is obviously true for the hyperbolic dimension).

Let $R^{n_{k}} f \rightarrow f$, and let $g$ be a limit of $R^{n_{k}-1} f$. Then $R g=f$, so that $\delta_{*}(g) \geq$ $\delta_{*}(f)$. On the other hand, by the lower semicontinuity of $\delta_{*}$ (Lemma 2.8) we have $\delta_{*}(g) \leq \lim \delta_{*}\left(R^{i}(f)\right) \leq \delta_{*}(f)$. Thus $\delta_{*}(f)=\delta_{*}(g)$, and Proposition 3.6 completes the proof.

\footnotetext{
${ }^{7}$ The lemma (and the proof) holds for rational maps whose postcritical set is not dense in the Julia set.
} 


\subsection{Conformal spectrum.}

Proposition 3.8. For any $\delta \geq \operatorname{HD}_{\text {hyp }}(J)$, there exists a unique $\delta$-conformal measure $\mu_{\delta}$. In particular, there always exists a unique 2-conformal measure $\mu_{2}$.

Proof. The uniqueness part follows from Theorem 2.9, so let us deal with the existence.

Let $S_{n}^{r}=\left\{\zeta: f^{n} \zeta=0\right.$ and $\left.\left|f^{k} \zeta\right| \geq r, k=0, \ldots, n-1\right\}$ and let $S^{r}=\bigcup S_{n}^{r}$. Let us consider the cut-off Poincaré series

$$
\Xi_{\delta}^{r}(0)=\sum_{n=0}^{\infty} \sum_{\zeta \in S_{n}^{r}} \frac{1}{\left|D f^{n}(\zeta)\right|^{\delta}} .
$$

Let us now consider a hyperbolic set $J^{r}$; see (2.3). By Lemma 2.11, it is contained in a hyperbolic repeller $Q^{r}$, and hence

$$
\delta_{\text {cr }}\left(f \mid J^{r}\right) \leq \delta_{\text {cr }}\left(f \mid Q^{r}\right)=\operatorname{HD}\left(Q^{r}\right) \leq \operatorname{HD}_{\text {hyp }}(J) .
$$

It follows that the cut-off Poincaré series is convergent for $\delta>\operatorname{HD}_{\text {hyp }}(J)$. Hence we can weigh the involved preimages of 0 by the terms of $\Xi_{\delta}^{r}(0)$ and consider the corresponding probability measure:

$$
\mu_{\delta}^{r}=\frac{1}{\Xi_{\delta}^{r}(0)} \sum_{n=0}^{\infty} \sum_{\zeta \in S_{n}^{r}} \frac{\delta_{\zeta}}{\left|D f^{n}(\zeta)\right|^{\delta}} .
$$

Now let $r \rightarrow 0\left(\delta>\delta_{*}\right.$ being fixed $)$. Then $\Xi_{\delta}^{r}(0) \rightarrow \infty$. To see this, consider the pre-renormalizations $f_{m}: \mathbb{U}^{m} \rightarrow \mathbb{V}^{m}$ and pick some $f_{m}$-preimage $\zeta_{m}$ of 0 . Since the derivatives $\left|D f_{m}\left(\zeta_{m}\right)\right|$ are bounded,

$$
\lim _{r \rightarrow 0} \Xi_{\delta}^{r}(0) \geq \sum_{m=0}^{\infty} \frac{1}{\left|D f_{m}\left(\zeta_{m}\right)\right|^{\delta}}=\infty,
$$

as was asserted.

Let us now consider a weak* limit $\mu_{\delta}$ of measures $\mu_{\delta}^{r}$ as $r \rightarrow 0$. We claim that this measure is $\delta$-conformal. Let us first verify this outside the critical point. Let $U$ be a small disk whose closure does not contain 0 and such that $f \mid U$ is injective. Then for sufficiently small $r>0$,

$$
f\left(S_{n}^{r} \cap U\right)=S_{n-1}^{r} \cap f(U),
$$

and hence

$$
\left.\mu_{\delta}^{r}|f(U)=| D f\right|^{\delta}\left(\mu_{\delta}^{r} \mid U\right) .
$$

Passing to the limit, we conclude that

$$
\left.\mu_{\delta}|f(U)=| D f\right|^{\delta}\left(\mu_{\delta} \mid U\right),
$$

provided $\mu_{\delta}(\partial U)=0$. Since there is a base of disks with the latter property, the measure $\mu_{\delta}$ is $\delta$-conformal everywhere outside 0 .

To complete the proof, we need to show that the critical value $f(0)$ does not carry any mass. Let us assume the contrary, and consider the maps $g_{m}=f_{m} \circ f^{-1}$ that carry $f(0)$ to $f_{m}(0)$ and $f\left(J_{m}\right)$ to $J_{m}$. A priori bounds imply that they have bounded distortion in a definite neighborhood of $f\left(J^{m}\right)$. Since diam $f\left(J^{m}\right) \asymp$ $\left(\operatorname{diam} J^{m}\right)^{2}$, we conclude that $\left|D g_{m}(f(0))\right| \asymp\left(\operatorname{diam} J^{m}\right)^{-1}$. Since $\mu_{\delta}$ is $\delta$-conformal outside 0 ,

$$
\mu_{\delta}\left(f_{m}(0)\right)=\left|D g_{m}(f(0))\right|^{\delta} \mu_{\delta}(f(0)) \asymp\left(\operatorname{diam} J^{m}\right)^{-\delta} \mu_{\delta}(f(0)) \rightarrow \infty \quad \text { as } m \rightarrow \infty,
$$


which is impossible since $\mu_{\delta}$ is a finite measure. The lemma is proved.

We will make use of the easy part of Denker-Urbański-Przytycki's Theorem:

Lemma 3.9. For any quadratic-like map $f, \operatorname{HD}_{\text {hyp }}(J) \leq \delta_{*}$.

Proof. Let us consider a $\delta$-conformal measure $\mu$ and a hyperbolic set $X \subset J$. We need to show that $\operatorname{HD}(X) \leq \delta$.

By a standard distortion argument using $\delta$-covariance of $\mu$, we obtain:

$$
\mu_{\delta}\left(\mathbb{D}_{r}(z)\right) \asymp r^{\delta}
$$

for all disks $\mathbb{D}_{r}(z)$ centered at $z \in X$ of radius $r \leq 1$.

By the Besikovich Covering Lemma, for any $\epsilon>0$, there exists a covering of $X$ with disks $\mathbb{D}_{r_{i}}\left(z_{i}\right)$ of radii $r_{i}<\epsilon$ with intersection multiplicity bounded by $N$. Then

$$
\sum r_{i}^{\delta} \asymp \sum \mu_{\delta}\left(\mathbb{D}_{r_{i}}\left(z_{i}\right)\right)^{\delta} \leq N \mu_{\delta}(X) .
$$

Hence $h_{\delta}(X)<\infty$, so that $\delta \geq \operatorname{HD}(X)$.

Theorem 3.10. For any Feigenbaum map, $\operatorname{HD}_{\text {hyp }}(J)=\delta_{*}=\delta_{\text {cr }}$.

Proof. We will show that $\operatorname{HD}_{\text {hyp }}(J) \leq \delta_{*} \leq \delta_{\text {cr }} \leq \operatorname{HD}_{\text {hyp }}(J)$.

The first inequality is the content of Lemma 3.9.

The middle inequality, $\delta_{*} \leq \delta_{\mathrm{cr}}$, holds since by Theorem 2.6 there exists a $\delta_{\mathrm{cr}^{-}}$ conformal measure, and by definition, $\delta_{*}$ is the minimum exponent of conformal measures on $J$.

To prove the last inequality, $\delta_{\text {cr }} \leq \operatorname{HD}_{\text {hyp }}(J)$, take any $\delta>\operatorname{HD}_{\text {hyp }}(J)$. Then by Proposition 3.8, there exists a $\delta$-conformal measure on $J$. By Lemma 3.5, it is dissipative. By Lemma 3.1, the Poincaré series $\Xi_{\delta}(z), z \in \mathbb{C} \backslash \mathcal{O}$, is convergent. By definition, $\delta_{\mathrm{cr}} \leq \delta$, and the conclusion follows.

Remark 3.1. Our exposition is organized in such a way that it does not explicitly use the constructive part of Theorem 2.7 of Denker-Urbański and Przytycki (which provides a minimal conformal measure $\mu_{*}$ ), so that along the lines we obtain a new proof of it (in the Feigenbaum setting).

3.5. Scaling at the critical point. Let $\mu$ be a $\delta$-conformal measure on the Julia set $J(f)$, and let

$$
\sigma=\lim _{r \rightarrow 0} \sup \frac{\log \mu\left(\mathbb{D}_{r}\right)}{\log r} .
$$

Lemma 3.11. If area $(J(f))=0$, then $\sigma \leq \delta$.

Proof. Let us consider a fundamental annulus $A^{1}=V^{1} \backslash U^{1}$ of the pre-renormalization $g$ of $f$, where $V^{1}$ and $U^{1}$ are domains constructed in $\S 2.7$. Then $\mu\left(A^{1}\right)>0$ since $A^{1} \cap J(f) \neq \emptyset$ and $\operatorname{supp} \mu=J(f)$. We also have $\mu\left(\partial A^{1}\right)=0 .{ }^{8}$ Given a point $z \in U^{1} \backslash J(g)$, there is a unique moment $n(z)$ such that $g^{n(z)}(z) \in A^{1}$. Since area $(J(g))=0, n(z)$ is well defined for Lebesgue a.e. $z \in U^{1}$.

Let us tile $\bar{A}^{1}$ by finitely many compact sets $\Delta_{i} \subset \bar{A}^{1}$ of positive $\mu$-measure that have boundary of zero $\mu$-measure, such that each $\Delta_{i}$ is contained in a Jordan domain $\tilde{\Delta}_{i} \subset \mathbb{V}^{1} \backslash \mathcal{O}(f)$.

\footnotetext{
${ }^{8}$ Indeed, we have $f^{n}\left(\partial A^{1}\right) \cap U^{1}=\emptyset, n \geq 0$, which implies that $\mu\left(\partial A^{1}\right)=0$ in both dissipative and conservative cases (since by Lemma 3.1, the orbit of $\mu$-a.e. $z \in J(f)$ accumulates on the critical point).
} 
Pulling the $\Delta_{i}$ back by the appropriate branches of $g^{-n(z)} \mid \tilde{\Delta}_{i}$, we obtain compact sets $\Pi_{j}$ covering Lebesgue almost all of $U^{1}$. Since $U^{1}$ is a nice domain, the $\Pi_{j}$ have pairwise disjoint interiors. Moreover, there exists $\tilde{\Pi}_{j} \ni \Pi_{j}$ such that if $g^{n_{j}}\left(\Pi_{j}\right)=$ $\Delta_{i}$, then $g^{n_{j}} \mid \tilde{\Pi}_{j}$ is a univalent map onto $\tilde{\Delta}_{i}$.

Since the $\tilde{\Pi}_{j}$ provide a definite Koebe space around $\Pi_{j}$, we can apply the Koebe Distortion Lemma to conclude that each $\Pi_{j}$ is mapped with bounded distortion by some $g^{n_{j}}$ onto the appropriate $\Delta_{i}$. Together with the $\delta$-covariance of $\mu$, this yields

$$
\mu\left(\Pi_{j}\right) \asymp \frac{\mu\left(\Delta_{i}\right)}{\left|D g^{n_{j}}(z)\right|^{\delta}} \asymp\left(\operatorname{diam} \Pi_{j}\right)^{\delta} .
$$

Take some $\epsilon>0$. Then there exist arbitrary small disks $\mathbb{D}_{r}$ such that $\mu\left(\mathbb{D}_{r}\right) \leq$ $r^{\sigma-\epsilon}$. Since the hyperbolic diameter of $\Pi_{j}$ in $\tilde{\Pi}_{j}$ is bounded and $0 \notin \tilde{\Pi}_{j}$, we have $\operatorname{diam} \Pi_{j} \leq C \operatorname{dist}\left(\Pi_{j}, 0\right)$. Hence there is a scaling factor $k>1$ independent of $r$ such that if $\Pi_{j} \cap \mathbb{D}_{r / k} \neq \emptyset$, then $\Pi_{j} \subset \mathbb{D}_{r}$. Since the $\Pi_{j}$ have disjoint interiors and have boundaries of zero $\mu$-measure, we conclude that

$$
\sum \mu\left(\Pi_{j}\right) \leq \mu\left(\mathbb{D}_{r}\right) \leq r^{\sigma-\epsilon},
$$

where the summation is taken over those $\Pi_{j}$ that intersect $\mathbb{D}_{r / k}$. Since these $\Pi_{j}$ cover Lebesgue almost all of $\mathbb{D}_{r / k}$,

$$
r^{2} \asymp \operatorname{area}\left(\mathbb{D}_{r / k}\right) \leq \sum \text { area } \Pi_{j} \asymp \sum\left(\operatorname{diam} \Pi_{j}\right)^{2} .
$$

Hence $r^{\delta} \leq C \sum\left(\operatorname{diam} \Pi_{j}\right)^{\delta} \asymp \sum \mu\left(\Pi_{j}\right)$. Comparing it with (3.3), we conclude that $\sigma-\epsilon \leq \delta$. Since it holds for any $\epsilon>0$, we are done.

\subsection{Hyperbolic dimension.}

Proposition 3.12. Let $\mu$ be a $\delta$-conformal measure on $J$. If $\operatorname{area}(J)=0$, then for any $z \in J, \underline{\mathrm{HD}}_{\mu}(z) \leq \delta$.

Proof. By Lemma 3.11, for any $\epsilon>0$ there exists a $c>0$ such that

$$
\mu\left(\mathbb{D}_{r}\right) \geq c r^{\delta+\epsilon}, \quad r \leq \operatorname{diam} J .
$$

Take a point $z \in J$. If the $\operatorname{orb}(z)$ does not accumulate on 0 , then it stays away from the postcritical set $\mathcal{O}$ (since $f \mid \mathcal{O}$ is minimal), and then obviously $\operatorname{HD}_{\mu}(z)=\delta$. If it does, then consider the first entries of the $\operatorname{orb}(z)$ to the domains $V^{m}$ from $\S 2.7$ and pull them back to $z$. We obtain a shrinking nest of neighborhoods $D^{m}$ of $z$ with bounded shape.

Let $l_{m}$ be such that $f^{l_{m}}\left(D^{m}\right)=V^{m}$. Then it follows from the properties (C2)(C3) of the domains $V^{m}$ that either $f^{l_{m}}\left(D^{m+1}\right) \subset U^{m}$ or $f^{l_{m}}\left(D^{m+1}\right) \subset\left(V^{m} \backslash U^{m}\right)$. In either case, by property (G2) of the domains $V^{m}$, there exists $\epsilon_{0}>0$ such that area $\left(f^{l_{m}}\left(D^{m+1}\right)\right) /$ area $\left(f^{l_{m}}\left(D^{m}\right)\right)<1-\epsilon_{0}$. Since $f^{l_{m}}: D^{m} \rightarrow V^{m}$ has bounded distortion (it admits a univalent extension onto $\mathbb{V}^{m}$ ), we conclude that there exists $\epsilon>0$ such that area $\left(D^{m+1}\right) / \operatorname{area}\left(D^{m}\right)<1-\epsilon$. So diam $\left(D^{m}\right)$ decays exponentially fast.

Since $\operatorname{diam}\left(V^{m}\right)>C \lambda^{m}$ for some $C>0, \lambda>0$, it follows that $\operatorname{diam}\left(D^{m}\right) \leq$ $c \operatorname{diam}\left(V^{m}\right)^{\kappa}$ for some $c>0, \kappa>0$. It easily follows from (3.4), bounded distortion, and $\delta$-covariance of $\mu$ that $\mu\left(D^{m}\right) \geq c\left(\operatorname{diam} D^{m}\right)^{\delta+\epsilon}$, and the conclusion follows.

Corollary 3.13. If area $(J)=0$, then $\operatorname{HD}(J)=\operatorname{HD}_{\text {hyp }}(J)$. 
Proof. By Theorem 2.7, it is enough to prove that for any $\delta$-conformal measure $\mu$ on $J, \operatorname{HD}(J) \leq \delta$. Take some $\epsilon>0$. By Proposition 3.12, any point $z \in J$ is a center of arbitrary small disks $\mathbb{D}_{r}(z)$ such that $\mu\left(\mathbb{D}_{r}(z)\right) \geq r^{\delta+\epsilon}$. By the Besicovich Covering Lemma, $J$ admits an arbitrary fine covering by such disks $D_{k}=\mathbb{D}\left(z_{k}, r_{k}\right)$ with intersection multiplicity at most $N$. Hence $\sum r_{k}^{\delta+\epsilon} \leq N \mu(J), h_{\delta}(J)<\infty$, and we are done.

Remark 3.2. This result also follows from Theorem 3.10 and the work of Bishop $[\mathrm{B}]$.

3.7. All dimensions and exponents are equal. Combining Theorem 3.10 with Corollary 3.13, we obtain:

Theorem 3.14. Assume that a Feigenbaum Julia set $J$ has zero area. Then

$$
\delta_{\text {cr }}=\delta_{*}=\operatorname{HD}_{\text {hyp }}(J)=\operatorname{HD}(J) .
$$

3.8. Continuity properties of the critical exponent. Let us formulate a couple of useful technical lemmas. Theorem 3.10 and Lemma 2.8 yield:

Corollary 3.15. The critical exponent $\delta_{\mathrm{cr}}(f)$ depends lower semicontinuously on the Feigenbaum map $f$.

The situation within one hybrid class is even better:

Lemma 3.16. The critical exponent $\delta_{\mathrm{cr}}(g)$ is continuous on any hybrid class $\mathcal{H}_{f}$. Moreover, it is uniformly continuous over compact subsets $K$ of quadratic-like germs: For any $\epsilon>0$ there exists $\sigma>0$ such that if $f, g \in K, g \in \mathcal{H}_{f}$ and $\operatorname{dist}(f, g)<\sigma$, then $\left|\delta_{\mathrm{cr}}(f)-\delta_{\mathrm{cr}}(g)\right|<\epsilon$.

Proof. If $f, g \in K, g \in \mathcal{H}_{f}$ and $\operatorname{dist}(f, g)<\sigma$, then $f$ and $g$ are conjugate by a $K$-qc homeomorphism $h$, where dilatation $K$ is close to 1 . This map is $\kappa$-Hölder continuous, with exponent $\kappa=1 / K$.

Let $f: \mathbb{U} \rightarrow \mathbb{V}$ and $\mathcal{O}=\mathcal{O}(f)$. Consider a round disk $D$ compactly contained in $\mathbb{V} \backslash \mathcal{O}$ centered around some point $x$. If $f^{m}(y)=x$, let $D_{y}$ be the component of $f^{-m}(x)$ containing $y$. Then by the Koebe Distortion Lemma, the maps $f^{m}$ : $D_{y} \rightarrow D$ and $g^{m}: h\left(D_{y}\right) \rightarrow h(D)$ have bounded distortion. Thus $\left|D f^{m}(y)\right| \asymp$ $\left(\operatorname{diam} D_{y}\right)^{-1}$ and $\left|D g^{m}(h(y))\right| \asymp\left(\operatorname{diam} h\left(D_{y}\right)\right)^{-1}$. This implies that

$$
M^{-1}\left|D f^{m}(y)\right|^{\kappa} \leq\left|D g^{m}(h(y))\right| \leq M\left|D f^{m}(y)\right|^{K} .
$$

Hence $K^{-1} \leq \delta_{\text {cr }}(f) / \delta_{\text {cr }}(g) \leq K$, and we are done.

\section{ReCURSIVE QUADRATIC ESTIMATES}

This is the central section of the paper. Here we compare the average Poincaré series on three renormalization levels, $l<m<n$. We start on the intermediate level $m$ and sort out the backward trajectories according to whether they go "in" to a deeper level $n$, or "out" to a shallower level $l$. The coefficients in this estimate depend on probabilities of going in and out that we are about to introduce.

Let $X_{m, n}, m<n$, be the set of points in $U^{m}$ that land in $V^{n}$ under iterates of $f_{m}$, and let $X_{n}=X_{0, n}$. Let $Y_{m, n}, m<n$, be the set of points in $A^{n}$ that never return to $V^{n}$ under iterates of $f_{m}$, and let $Y_{n}=Y_{0, n}$. We can now define the following quantities: 
- The probability of going from the disk $U^{m}$ to a deeper disk $V^{n}$

$$
\eta_{m, n} \equiv \eta_{m, n}(f)=\frac{\left|X_{m, n}\right|}{\left|U^{m}\right|}, \quad \eta_{n}=\eta_{0, n},
$$

- the probability of going from the annulus $A^{n}$ straight to the shalower annulus $A^{m}$

$$
\xi_{m, n} \equiv \xi_{m, n}(f)=\frac{\left|Y_{m, n}\right|}{\left|A^{n}\right|}, \quad \xi_{n}=\xi_{0, n},
$$

- and the scaling factors

$$
\rho_{m, n} \equiv \rho_{m, n}(f)=\frac{\left|U^{n}\right|}{\left|U^{m}\right|} .
$$

Let us start with a couple of relations between these quantities:

Lemma 4.1. Let $f$ be a Feigenbaum map. There exists $C>0$ only depending on the geometric bounds and $C_{0}>0$, depending also on the combinatorial bounds, such that

$$
\max \left\{C_{0}^{-1}, 1-C_{0} \xi_{m, n}\right\} \leq \frac{\eta_{m, n+1}}{\eta_{m, n}} \leq 1-C^{-1} \xi_{m, n} .
$$

Proof. We may assume that $m=0$. The first landing map $\phi: X_{n} \rightarrow V^{n}$ sends each component of $X_{n}$ univalently onto $V^{n}$ with bounded distortion. Clearly, $\phi^{-1}\left(Y_{n}\right) \cap$ $X_{n+1}=\emptyset$, so that

$$
\frac{\eta_{n+1}}{\eta_{n}}=p\left(X_{n+1} \mid X_{n}\right) \leq 1-C^{-1} \xi_{n} .
$$

Notice that $\left|V^{n+1}\right| /\left|V^{n}\right|>q$, where $q$ also depends on the combinatorial bounds, so we get

$$
\frac{\eta_{n+1}}{\eta_{n}}>C_{0}^{-1}
$$

To conclude the remaining inequality, it is enough to show that $p\left(X_{n+1} \mid V^{n}\right)>$ $1-C_{0} \xi_{n}$. Let $S^{n}$ be the set of points in $V^{n}$ which land in $A^{n}$ and later return to $V^{n}$, and let $W^{n}$ be the set of connected components of $S^{n}$ which are not contained in $V^{n+1}$. Then $\left|W^{n}\right| /\left|V^{n}\right|<1-q$, where $q$ also depends on the combinatorial bounds. We set $\psi: W^{n} \rightarrow V^{n}$ to be the composition of the first landing to $A^{n}$ followed by the first return to $V^{n}$. The restriction of $\psi$ to each connected component of $W^{n}$ is a univalent map onto $V^{n}$, moreover any iterate of $\psi$ has bounded distortion by the Koebe Distortion Lemma (the restriction of $\psi$ to each component of $W^{n}$ extends to a univalent map onto $\left.\mathbb{V}^{n}\right)$. Since $\left|W^{n}\right| /\left|V^{n}\right|<1-q$, we have $\left|\psi^{-k}\left(W^{n}\right)\right| \leq e^{-q k}\left|V^{n}\right|$.

Since almost every $x \in V^{n}$ either escapes through $Y_{n}$ or eventually passes through $V^{n+1}$, we have

$$
\frac{\left|V^{n} \backslash X_{n+1}\right|}{\left|V^{n}\right|} \leq \sum_{k=0}^{\infty} \frac{\left|\psi^{-k}\left(Y_{n}\right)\right|}{\left|V^{n}\right|} \leq C \sum_{k=0}^{\infty} e^{-q k} \xi_{n} \leq C_{0} \xi_{n} .
$$

Let $\Xi^{[j]}$ be the Poincaré series $\Xi$ truncated at level $j$, so that summation in (1.1) is taken over $n \leq j$ instead of all $n$. Let

$$
\omega_{m, n}(f, \delta) \equiv \omega_{m, n}(\delta)=\frac{1}{\left|A^{n}\right|} \int_{A^{n}} \Xi_{\delta}\left(f_{m}, x\right) d x, \quad \omega_{n}=\omega_{0, n} .
$$


We will use $\omega^{[j]}$ to denote the truncation of $\omega$ at level $j$, that is, taking the integral of $\Xi^{[j]}$ instead of $\Xi$.

Our key estimates are presented in Lemmas 4.2 and 4.4.

Lemma 4.2. Let $f$ be a Feigenbaum map. Then for any $0 \leq l<m<n$, there exists $C>0$, only depending on the geometric bounds, and $\epsilon>0$, depending on the geometric and combinatorial bounds, and on $n-l, m-l$, such that if $2-\epsilon \leq \delta \leq 2$, then

$$
\begin{gathered}
\omega_{l, m}^{[j+1]}(\delta) \leq C \\
\rho_{l, m} \frac{\eta_{l, m}}{\rho_{l, m}}+\left(1-C^{-1}\left(\eta_{m, n}+\xi_{l, m}\right)\right) \omega_{l, m}^{[j]}(\delta)+C \rho_{m, n} \xi_{m, n} \omega_{l, n}^{[j]}(\delta), \\
\omega_{l, n}^{[j+1]}(\delta) \leq C\left(\frac{\eta_{l, m}}{\rho_{l, m}}+\omega_{l, m}^{[j]}(\delta)\right) \omega_{m, n}^{[j]}(\delta) .
\end{gathered}
$$

Let $\tau_{m, n}, m<n$, be the infimum of $\left|D f_{m}^{k}(x)\right|$ over all $x \in U^{m}, k \geq 0$, such that $f_{m}^{k}(x) \in V^{n}$ and $f_{m}^{j}(x) \notin V^{n}, 0 \leq j \leq k$, and let $\tau_{n}=\tau_{0, n}$. Let $v_{m, n}, m<n$ be the infimum of $\left|D f_{m}^{k}(x)\right|$ over all $x \in A^{n}, k \geq 1$, such that $f_{m}^{k}(x) \in A^{m}$ and $f_{m}^{j}(x) \notin A^{m} \cup A^{n}, 1 \leq j \leq k$, and let $v_{n}=v_{0, n}$. Let us show that the product $\tau v$ is exponentially large in terms of $n-m$.

Lemma 4.3. Let $f$ be a Feigenbaum map. Then for every $0 \leq m<n$, there exist $C>0, \theta<1$, only depending on the geometric bounds, such that for every $m<n$ we have

$$
\tau_{m, n} v_{m, n} \geq C^{-1} \theta^{m-n} .
$$

Proof. We may assume that $m=0$. Let $f^{k}(x)$ be the first landing of $x$ in $V^{n}$. Then there exists a domain $D \subset U$ containing $x$ such that $f^{k}: D \rightarrow V^{n}$ is univalent and has bounded distortion. Now let $z \in A^{n}$ and let $y=\left(f^{k} \mid D\right)^{-1}(z)$. We claim that if $f^{s}(z) \in A$, then $\left|D f^{k+s}(y)\right| \geq C^{-1} \theta^{-n}$, which implies the result (notice that $\left|D f^{k}(x)\right|\left|D f^{s}(z)\right| \geq C^{-1}\left|D f^{k+s}(y)\right|$ by bounded distortion of $\left.f^{k} \mid D\right)$.

To see this, let $W$ be the complement of the postcritical set in some definite neighborhood of $V$, and consider the Poincaré metric $\|\cdot\|_{W}$ on $W$. Since $A$ is away from the postcritical set, we have

$$
\left|D f^{k+s}(y)\right| \geq C^{-1}\left\|D f^{k+s}(y)\right\|_{W} .
$$

It follows from the Schwarz Lemma that $f: U \rightarrow V$ does not contract $\|\cdot\|_{W}$, so

$$
\left\|D f\left(f^{j}(y)\right)\right\|_{W} \geq 1, \quad 0 \leq j \leq k+s-1 .
$$

Closer inspection (see [Mc3, Prop. 6.9]) shows that whenever $f^{j}(y) \in A^{i}$ with $1 \leq i \leq n$ we have $\left\|D f\left(f^{j}(y)\right)\right\|_{W} \geq \theta^{-1}$. Thus

$$
\left\|D f^{k+s}(y)\right\|_{W} \geq \theta^{-n} .
$$

Lemma 4.4. Let $f$ be a Feigenbaum map. Then for every $0 \leq l<m<n$, there exist $C>0$, only depending on the geometric bounds, such that if $\delta \leq 2$, then

$$
\begin{aligned}
& \omega_{l, m}(\delta) \geq C^{-1} \tau_{l, m}^{2-\delta} \frac{\eta_{l, m}}{\rho_{l, m}}+\max \left\{1-C\left(\eta_{m, n}+\xi_{l, m}\right), 0\right\} \omega_{l, m}(\delta) \\
&+C^{-1} v_{m, n}^{2-\delta} \xi_{m, n} \rho_{m, n} \omega_{l, n}(\delta), \\
& \omega_{l, n}(\delta) \geq C^{-1}\left(\tau_{l, m}^{2-\delta} \frac{\eta_{l, m}}{\rho_{l, m}}+\omega_{l, m}(\delta)\right) \omega_{m, n}(\delta) .
\end{aligned}
$$


4.1. Proof of Lemmas 4.2 and 4.4. We may assume that $l=0$. To simplify notation, truncation of the Poincaré series (labeled by $j$ or $j+1$ ) will be implicit in what follows.

We define $Z_{m, n}$ as the set of points in $A^{m}$ that do not return to $A^{m}$ before passing through $A^{n}$. We let

$$
\kappa_{m, n}=\frac{\left|Z_{m, n}\right|}{\left|A^{m}\right|}
$$

Notice that

$$
\kappa_{m, n} \asymp \xi_{m}+\eta_{m, n} .
$$

In this section, an orbit (under iteration of $f_{r}$ ) will denote a sequence $\left(x_{0}, \ldots, x_{k}\right)$, $k \geq 0$, where $x_{k} \in V^{r}$ and $f_{r}\left(x_{i}\right)=x_{i+1}, i<k$. Unless explicitly stated otherwise, the orbits considered will be orbits under iteration of $f$.

An orbit will be said to be trivial if its length is 0 .

Given a family $\mathcal{F}$ of orbits $\left(x_{0}, \ldots, x_{k}\right)$ under iteration of some $f_{r}$, we define a function $\mathbb{C} \rightarrow[0, \infty]$

$$
\Xi_{\delta}(\mathcal{F})(z)=\sum_{\left(x_{0}, \ldots, x_{k}=z\right) \in \mathcal{F}}\left|D f_{r}^{k}\left(x_{0}\right)\right|^{-\delta}
$$

(notice that we do not make $f_{r}$ explicit in this notation).

We shall need some special notation for certain families of orbits. By $D \leftarrow E$, we will understand the family of orbits $\left(x_{0}, \ldots, x_{k}\right)$ with $x_{0} \in E$ and $x_{k} \in D$. The family of orbits $\left(x_{0}, \ldots, x_{k}\right)$ with $x_{0} \in E, x_{k} \in D$ and $x_{1}, \ldots, x_{k-1} \in S$ will be denoted $D \overleftarrow{S} E$. A "plus sign" over the arrow will indicate that only non-trivial orbits are considered. The justaposition of arrows will denote composition in the natural way. Thus

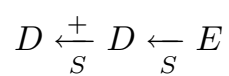

denotes the family of orbits $\left(x_{0}, \ldots, x_{k}\right)$, with $x_{0} \in E, x_{k} \in D$, and such that there exists $0 \leq i<k$ such that $x_{i} \in D$ and $x_{1}, \ldots, x_{i-1}, x_{i+1}, \ldots, x_{k-1} \in S$.

When considering orbits under some $f_{r}$ other than $f$, this will be indicated above the arrow, for instance, $D \stackrel{f_{r}}{\stackrel{S}{S}} E$ or $D \stackrel{f_{r},+}{\longleftarrow} E$.

Given a function $\phi: \mathbb{C} \rightarrow[0, \infty]$ and a measurable set $N \subset \mathbb{C}$, we let

$$
I(\phi \mid N)=\int_{N} \phi(x) d x
$$

and if $|N|>0$, we let

$$
E(\phi \mid N)=\frac{1}{|N|} I(\phi \mid N)
$$

More generally, if $\mu$ is a measure on $\mathbb{C}$ with $\mu(\mathbb{V})<\infty$, we let

$$
E_{\mu}(\phi \mid N)=\frac{1}{\mu(N)} \int_{N} \phi(x) d \mu(x)
$$

whenever $\mu(N)>0$.

In this notation, we have for instance

$$
\omega_{m}(\delta)=E\left(\Xi_{\delta}\left(A^{m} \leftarrow U\right) \mid A^{m}\right) .
$$


Let us say that a family $\mathcal{F}$ of orbits is hyperbolic if $\left(x_{0}, \ldots, x_{k}\right) \in \mathcal{F}$ implies that $\left|D f^{k}\left(x_{0}\right)\right| \geq C \lambda^{k}, C>0, \lambda>1$. Notice that $\mathcal{F}$ is hyperbolic if and only if there exists a neighborhood $W$ of 0 such that if $\left(x_{0}, \ldots, x_{k}\right) \in \mathcal{F}$, then $x_{0}, \ldots, x_{k-1} \notin W$.

Let $\delta_{\mathcal{F}}$ be the infimum over all $\delta$ such that $\Xi_{\delta}(\mathcal{F})$ is uniformly bounded. By Corollary $2.14, \delta_{\mathcal{F}}<2$ if $\mathcal{F}$ is hyperbolic.

Lemma 4.5. With $\delta_{\mathcal{F}}$ defined as above, we have:

(1) The map $\delta \mapsto \sup \Xi_{\delta}(\mathcal{F})$ is a continuous convex function of $\delta>\delta_{\mathcal{F}}$.

(2) Let $\mu$ be a finite measure on $\mathbb{V}$, and let

$$
\nu_{\delta}=\int \sum_{\left(x_{0}, \ldots, x_{k}=x\right) \in \mathcal{F}} \frac{\delta_{x_{0}}}{\left|D f^{k}\left(x_{0}\right)\right|^{\delta}} d \mu(x)
$$

which is a finite measure for $\delta>\delta_{\mathcal{F}}$. Then for any Borelian set $N, \delta \mapsto$ $\nu_{\delta}(N)$ is a continuous convex function of $\delta>\delta_{\mathcal{F}}$. In particular $\delta \mapsto \nu_{\delta}$, $\delta>\delta_{\mathcal{F}}$ is continuous in the weak $k^{*}$ topology.

Proof. Follows immediately from the convexity of $\delta \mapsto x^{-\delta}$.

In the most common application of the previous lemma, we will take $\mu$ as Lebesgue and $\mathcal{F}$ as a set of hyperbolic first returns or landings, say $A^{m} \stackrel{+}{U \backslash V^{n}} A^{m}$. In this case, the measure $\nu_{2}$ is the restriction of Lebesgue measure to the set of points that land or return.

4.1.1. Proof of (4.9). Let

$$
B \equiv B^{m, n}=U \backslash\left(A^{m} \cup A^{n}\right) .
$$

We clearly have

$$
\omega_{m}(\delta)=E\left(\Xi_{\delta}\left(A^{m} \leftarrow U\right) \mid A^{m}\right)=1+E\left(\Xi_{\delta}\left(A^{m} \stackrel{+}{\longleftarrow} U\right) \mid A^{m}\right),
$$

where the 1 accounts for the trivial orbits.

Since $U$ is the disjoint union of $A^{n}, A^{m}$ and $B$, we can split $A^{m} \stackrel{+}{\longleftarrow}$, the set of non-trivial orbits that terminate in $A^{m}$ (under iteration by $f$ ), in three groups:

(1) $A^{m} \overleftarrow{B}_{B} B$ which consists of orbits $\left(x_{0}, \ldots, x_{k}\right)$ terminating in $A^{m}$ such that $x_{0}, \ldots, x_{k-1}$ do not intersect $A^{m} \cup A^{n}$,

(2) $A^{m} \overleftarrow{B}_{B} A^{n} \leftarrow U$ which consists of orbits $\left(x_{0}, \ldots, x_{k}\right)$ such that $x_{0}, \ldots, x_{k-1}$ intersects $A^{n} \cup A^{m}$ and the last such intersection happens in $A^{n}$,

(3) $A^{m} \underset{B}{\stackrel{+}{t}} A^{m} \leftarrow U$ which consists of non-trivial orbits $\left(x_{0}, \ldots, x_{k}\right)$ such that $x_{0}, \ldots, x_{k-1}$ intersects $A^{m} \cup A^{n}$ and the last such intersection happens in $A^{m}$.

Together with (4.24), this decomposition gives

$$
\begin{aligned}
\omega_{m}(\delta)= & 1+E\left(\Xi_{\delta}\left(A^{m} \underset{B}{\longleftarrow} B\right) \mid A^{m}\right)+E\left(\Xi_{\delta}\left(A^{m} \overleftarrow{B}_{B} A^{n} \leftarrow U\right) \mid A^{m}\right) \\
& +E\left(\Xi_{\delta}\left(A^{m} \underset{B}{\stackrel{+}{\leftarrow}} A^{m} \leftarrow U\right) \mid A^{m}\right) .
\end{aligned}
$$

Notice that

$$
I\left(\Xi_{2}\left(A^{m} \underset{B}{\longleftarrow} B\right) \mid A^{m}\right)
$$


is the area of the set of points in $B$ that land in $A^{m}$ before passing through $V^{n}$, which is certainly smaller than $\eta_{m}|U|$. Thus we have

$$
E\left(\Xi_{2}\left(A^{m} \overleftarrow{B}_{B} B\right) \mid A^{m}\right) \leq \eta_{m} \frac{|U|}{\left|A^{m}\right|}=\frac{\eta_{m}}{\rho_{m}} \frac{\left|U^{m}\right|}{\left|A^{m}\right|} .
$$

By hyperbolicity of the set of orbits that avoid $V^{n}$, we have

$$
E\left(\Xi_{\delta}\left(A^{m} \overleftarrow{B}_{B} B\right) \mid A^{m}\right) \leq C \frac{\eta_{m}}{\rho_{m}}, \quad \delta \approx 2,
$$

by continuity of the left hand side in $\delta \approx 2$.

We have the obvious estimate

$$
E\left(\Xi_{\delta}\left(A^{m} \overleftarrow{B} A^{n} \leftarrow U\right) \mid A^{m}\right) \leq E\left(\Xi_{\delta}\left(A^{m} \underset{B}{\leftarrow} A^{n}\right) \mid A^{m}\right) \sup _{A^{n}} \Xi_{\delta}\left(A^{n} \leftarrow U\right)
$$

By bounded oscillation of the Poincaré series in $A^{n}$, we have

$$
\inf _{A^{n}} \Xi_{\delta}\left(A^{n} \leftarrow U\right) \asymp \sup _{A^{n}} \Xi_{\delta}\left(A^{n} \leftarrow U\right) \asymp E\left(\Xi_{\delta}\left(A^{n} \leftarrow U\right) \mid A^{n}\right)=\omega_{n}(\delta) .
$$

Moreover,

$$
I\left(\Xi_{2}\left(A^{m} \underset{B}{\longleftarrow} A^{n}\right) \mid A^{m}\right)
$$

is the area of the set of points in $A^{n}$ that land in $A^{m}$ before passing through $V^{n}$. This is precisely $\left|A^{n}\right| \xi_{m, n}$, so

$$
E\left(\Xi_{2}\left(A^{m} \underset{B}{\longleftarrow} A^{n}\right) \mid A^{m}\right)=\xi_{m, n} \frac{\left|A^{n}\right|}{\left|A^{m}\right|}=\xi_{m, n} \rho_{m, n} \frac{\left|U^{m}\right|}{\left|A^{m}\right|} \frac{\left|A^{n}\right|}{\left|U^{n}\right|} \leq C \xi_{m, n} \rho_{m, n} .
$$

By hyperbolicity of the set of orbits avoiding $U^{n}$, we get

$$
E\left(\Xi_{\delta}\left(A^{m} \overleftarrow{B} A^{n}\right) \mid A^{m}\right) \leq C \xi_{m, n} \rho_{m, n}, \quad \delta \approx 2 .
$$

Estimates (4.29), (4.30) and (4.33) imply

$$
E\left(\Xi_{\delta}\left(A^{m} \overleftarrow{B} A^{n} \leftarrow U\right) \mid A^{m}\right) \leq C \xi_{m, n} \rho_{m, n} \omega_{n}(\delta), \quad \delta \approx 2
$$

Let us estimate the last term of (4.25). We have the identity

$$
\begin{aligned}
\frac{E\left(\Xi_{2}\left(A^{m} \stackrel{+}{{ }_{B}} A^{m} \leftarrow U\right) \mid A^{m}\right)}{E\left(\Xi_{2}\left(A^{m} \leftarrow U\right) \mid A^{m}\right)} & =\frac{E\left(\Xi_{2}\left(A^{m} \backslash Z_{m, n} \leftarrow U\right) \mid A^{m}\right)}{E\left(\Xi_{2}\left(A^{m} \leftarrow U\right) \mid A^{m}\right)} \\
& =1-\frac{E\left(\Xi_{2}\left(Z_{m, n} \leftarrow U\right) \mid A^{m}\right)}{E\left(\Xi_{2}\left(A^{m} \leftarrow U\right) \mid A^{m}\right)} .
\end{aligned}
$$

By bounded oscillation of Poincaré series, we have

$$
\frac{E\left(\Xi_{\delta}\left(Z_{m, n} \leftarrow U\right) \mid A^{m}\right)}{E\left(\Xi_{\delta}\left(A^{m} \leftarrow U\right) \mid A^{m}\right)} \asymp \kappa_{m, n} .
$$

By hyperbolicity of the set of orbits that do not pass through $V^{n}$, we have

$$
\begin{aligned}
E\left(\Xi_{\delta}\left(A^{m} \underset{B}{\stackrel{+}{t}} A^{m} \leftarrow U\right) \mid A^{m}\right) & \leq\left(1-C^{-1} \kappa_{m, n}\right) E\left(\Xi_{\delta}\left(A^{m} \leftarrow U\right) \mid A^{m}\right) \\
& =\left(1-C^{-1} \kappa_{m, n}\right) \omega_{m}(\delta), \quad \delta \approx 2 .
\end{aligned}
$$

Plugging (4.28), (4.34) and (4.37) in (4.25), we get

$$
\omega_{m}(\delta)=1+C \frac{\eta_{m}}{\rho_{m}}+\left(1-C^{-1} \kappa_{m, n}\right) \omega_{m}(\delta)+C \xi_{m, n} \rho_{m, n} \omega_{n}(\delta), \quad \delta \approx 2,
$$

which implies (4.9) (notice that $\eta_{m} \geq \rho_{m}$, so the 1 can be put inside $C \eta_{m} / \rho_{m}$ ). 
4.1.2. Proof of (4.10). Let us split $A^{n} \leftarrow U$ into two groups of orbits:

(1) $A^{n} \overleftarrow{U \backslash A^{m}} U \backslash A^{m}$

(2) $A^{n} \underset{U \backslash A^{m}}{\longleftarrow} A^{m} \leftarrow U$.

We further split $A^{n} \overleftarrow{U \backslash A^{m}} U \backslash A^{m}$ into two groups:

(1a) $A^{n} \overleftarrow{U \backslash A^{m}} U^{m}$,

(1b) $A^{n} \underset{U \backslash A^{m}}{\longleftarrow} U^{m} \overleftarrow{U \backslash V^{m}} U \backslash V^{m}$.

Notice that we can write

$$
\left(A^{n} \underset{U \backslash A^{m}}{\overleftarrow{ }} A^{m}\right)=\left(A^{n} \underset{U \backslash A^{m}}{\longleftarrow} U^{m} \underset{U \backslash V^{m}}{\overleftarrow{ }} A^{m}\right)
$$

Thus we have

$$
\begin{aligned}
\omega_{n}(\delta)=E\left(\Xi_{\delta}\left(A^{n} \leftarrow U\right) \mid A^{n}\right)= & E\left(\Xi_{\delta}\left(A^{n} \overleftarrow{U \backslash A^{m}} U^{m}\right) \mid A^{n}\right) \\
& +E\left(\Xi_{\delta}\left(A^{n} \overleftarrow{U \backslash A^{m}} U^{m} \overleftarrow{U \backslash V^{m}} U \backslash V^{m}\right) \mid A^{n}\right) \\
& +E\left(\Xi_{\delta}\left(A^{n} \overleftarrow{U \backslash A^{m}} U^{m} \overleftarrow{U \backslash V^{m}} A^{m} \leftarrow U\right) \mid A^{n}\right) .
\end{aligned}
$$

This gives the estimate

$$
\begin{aligned}
\frac{E\left(\Xi_{\delta}\left(A^{n} \leftarrow U\right) \mid A^{n}\right)}{E\left(\Xi_{\delta}\left(A^{n} \overleftarrow{U \backslash A^{m}} U^{m}\right) \mid A^{n}\right)} \leq & 1+\sup _{U^{m}} \Xi_{\delta}\left(U^{m} \overleftarrow{U \backslash V^{m}} U \backslash V^{m}\right) \\
& +\sup _{U^{m}} \Xi_{\delta}\left(U^{m} \overleftarrow{\overleftarrow{U \backslash V^{m}}} A^{m} \leftarrow U\right) .
\end{aligned}
$$

Since $f_{m}$ is the first return map from $U^{m}$ to $V^{m}$, there is a natural correspondence between the orbits of $A^{n} \underset{U \backslash A^{m}}{\overleftarrow{ }} U^{m}$ and $A^{n} \stackrel{f_{m}}{\longleftarrow} U^{m}$. It follows that

$$
\Xi_{\delta}\left(A^{n} \underset{U \backslash A^{m}}{\longleftarrow} U^{m}\right)=\Xi_{\delta}\left(A^{n} \stackrel{f_{m}}{\longleftarrow} U^{m}\right)
$$

thus

$$
E\left(\Xi_{\delta}\left(A^{n} \underset{U \backslash A^{m}}{\longleftarrow} U^{m}\right) \mid A^{n}\right)=E\left(\Xi_{\delta}\left(A^{n} \stackrel{f_{m}}{\longleftarrow} U^{m}\right) \mid A^{n}\right)=\omega_{m, n}(\delta) .
$$

By the Markov property ${ }^{9}$ of the first landing map to $V^{m}$, we have

$$
\begin{aligned}
\sup _{U^{m}} \Xi_{2}\left(U^{m} \overleftarrow{U \backslash V^{m}} U \backslash V^{m}\right) & \asymp \inf _{U^{m}} \Xi_{2}\left(U^{m} \overleftarrow{U \backslash V^{m}} U \backslash V^{m}\right) \\
& \asymp E\left(\Xi_{2}\left(U^{m} \overleftarrow{U \backslash V^{m}} U \backslash V^{m}\right) \mid U^{m}\right) \asymp \frac{\eta_{m}}{\rho_{m}} .
\end{aligned}
$$

By hyperbolicity of the set of orbits avoiding $U^{m}$,

$$
\sup _{U^{m}} \Xi_{\delta}\left(U^{m} \underset{U \backslash V^{m}}{\longleftarrow} U \backslash V^{m}\right) \leq C \frac{\eta_{m}}{\rho_{m}}, \quad \delta \approx 2 .
$$

Similarly,

$$
\begin{aligned}
\sup _{U^{m}} \Xi_{2}\left(U^{m} \overleftarrow{U \backslash V^{m}} A^{m}\right) & \asymp \inf _{U^{m}} \Xi_{2}\left(U^{m} \overleftarrow{U \backslash V^{m}} A^{m}\right) \\
& \asymp E\left(\Xi_{2}\left(U^{m} \overleftarrow{U \backslash V^{m}} A^{m}\right) \mid U^{m}\right) \asymp 1,
\end{aligned}
$$

\footnotetext{
${ }^{9}$ Let us remark that one does not need to use the Markov property to conclude the upper bound in estimates (4.44) and (4.46).
} 
and by hyperbolicity of the set of orbits avoiding $U^{m}$, we get

$$
E\left(\Xi_{\delta}\left(U^{m} \overleftarrow{U \backslash U^{m}} A^{m}\right) \mid U^{m}\right) \leq C, \quad \delta \approx 2 .
$$

Estimate (4.47) implies

$$
\sup _{U^{m}} \Xi_{\delta}\left(U^{m} \underset{U \backslash V^{m}}{\longleftarrow} A^{m} \leftarrow U\right) \leq C \sup _{A^{m}} \Xi_{\delta}\left(A^{m} \leftarrow U\right), \quad \delta \approx 2,
$$

and by bounded oscillation of the Poincaré series in $A^{m}$, we get

$$
\sup _{U^{m}} \Xi_{\delta}\left(U^{m} \underset{U \backslash V^{m}}{\longleftarrow} A^{m} \leftarrow U\right) \leq C \omega_{m}(\delta), \quad \delta \approx 2 .
$$

Plugging (4.45) and (4.49) in (4.41) we get

$$
\frac{E\left(\Xi_{\delta}\left(A^{n} \leftarrow U\right) \mid A^{n}\right)}{E\left(\Xi_{\delta}\left(A^{n} \overleftarrow{\longleftarrow \backslash A^{m}} U^{m}\right) \mid A^{n}\right)} \leq 1+C \frac{\eta_{m}}{\rho_{m}}+C \omega_{m}(\delta), \quad \delta \approx 2 .
$$

This and (4.43) imply (4.10).

4.1.3. Proof of (4.12). It is easy to see that if $x \in A^{n}$ and $f^{k}(x) \in A^{m}$, then $\left|D f^{k}(x)\right| \geq C^{-1} \theta^{m-n}$ for some $\theta<1$. This follows from the Schwarz Lemma and the Koebe Distortion Lemma; see Lemma 4.1 for an analogous estimate.

For simplicity, we will assume that if $x \in A^{m}$ and $f^{k}(x) \in A^{m}$, then $\left|D f^{k}(x)\right| \geq 1$ (a slightly different argument allows us to deal with the general case) ${ }^{10}$ This assumption can be made without loss of generality; see Remark A.1.

As in the proof of (4.9), we shall use the identity (4.25).

Notice that the argument for the upper bound on $E\left(\Xi_{2}\left(A^{m} \overleftarrow{B}_{B} B\right) \mid A^{m}\right)$ (see (4.27)) also implies a lower bound of the same order,

$$
E\left(\Xi_{2}\left(A^{m} \underset{B}{\longleftarrow} B\right) \mid A^{m}\right) \geq C^{-1} \frac{\eta_{m}}{\rho_{m}} .
$$

Similarly, the argument of (4.32) gives the bound

$$
E\left(\Xi_{2}\left(A^{m} \underset{B}{\leftarrow} A^{n}\right) \mid A^{m}\right) \geq C^{-1} \xi_{m, n} \rho_{m, n} .
$$

Thus

$$
\begin{gathered}
E\left(\Xi_{\delta}\left(A^{m} \underset{B}{\longleftarrow} B\right) \mid A^{m}\right) \geq C^{-1} \frac{\eta_{m}}{\rho_{m}} \tau_{m}^{2-\delta} \\
E\left(\Xi_{\delta}\left(A^{m} \underset{B}{\longleftarrow} A^{n}\right) \mid A^{m}\right) \geq C^{-1} \xi_{m, n} \rho_{m, n} v_{m, n}^{2-\delta}
\end{gathered}
$$

\footnotetext{
${ }^{10}$ Let $W$ be the complement of the postcritical set of $f$ in some definite neighborhood of $V$ and let $\|\cdot\|_{W}$ denote the Poincaré metric on $W$. By the Schwarz Lemma, $f: U \rightarrow V$ expands $\|\cdot\|_{W}$. Let $\|\cdot\|_{*}$ denote the metric on $\mathbb{C}$ which is equal to the usual Euclidean metric outside $\bigcup A^{r}$ and in each $A^{r}$ is given by $\|\cdot\|$, suitably rescaled so that the $\|\cdot\|_{*}$ area of $A^{r}$ coincides with the usual Euclidean area of $A^{r}$. Since the $A^{r}$ are away from the postcritical set, the metric $\|\cdot\|_{*}$ is comparable to the usual Euclidean metric everywhere. Moreover, we get improved inequality that if $x \in A^{m}$ and $f^{k}(x) \in A^{m}$, then $\left\|D f^{k}(x)\right\|_{*} \geq 1$ (instead of $\left|D f^{k}(x)\right|>C^{-1}$ ). If one defines the Poincaré series, $\omega_{m, n}, \eta_{m, n}, \xi_{m, n}, \ldots$ with respect to the metric $\|\cdot\|_{*}$, the resulting objects are comparable to the Euclidean ones. Thus, it is enough to prove (4.12) for those modified objects, which can be done with the argument given below.
} 
Recall equality (4.35). The expansion of the Euclidean metric along orbits of $A^{m} \underset{B}{\stackrel{+}{+}} A^{m}$ implies that

$$
\begin{aligned}
\frac{E\left(\Xi_{\delta}\left(A^{m} \stackrel{+}{\stackrel{+}{B}} A^{m} \leftarrow U\right) \mid A^{m}\right)}{E\left(\Xi_{\delta}\left(A^{m} \leftarrow U\right) \mid A^{m}\right)} & \geq \frac{E\left(\Xi_{\delta}\left(A^{m} \backslash Z_{m, n} \leftarrow U\right) \mid A^{m}\right)}{E\left(\Xi_{\delta}\left(A^{m} \leftarrow U\right) \mid A^{m}\right)} \\
& =1-\frac{E\left(\Xi_{\delta}\left(Z_{m, n} \leftarrow U\right) \mid A^{m}\right)}{E\left(\Xi_{\delta}\left(A^{m} \leftarrow U\right) \mid A^{m}\right)},
\end{aligned}
$$

for $\delta \leq 2$. By (4.36), we get

$$
\begin{aligned}
E\left(\Xi_{\delta}\left(A^{m} \underset{B}{\stackrel{+}{+}} A^{m} \leftarrow U\right) \mid A^{m}\right) & \geq\left(1-C \kappa_{m, n}\right) E\left(\Xi_{\delta}\left(A^{m} \leftarrow U\right) \mid A^{m}\right) \\
& =\left(1-C \kappa_{m, n}\right) \omega_{m}(\delta) .
\end{aligned}
$$

Plugging (4.53), (4.54) and (4.56) in (4.25), we obtain (4.12).

4.1.4. Proof of (4.13). We will use the identity (4.40), which leads to the lower bound (analogous to (4.41))

$$
\begin{aligned}
\frac{E\left(\Xi_{\delta}\left(A^{n} \leftarrow U\right) \mid A^{n}\right)}{E\left(\Xi_{\delta}\left(A^{n} \overleftarrow{U \backslash A^{m}} U^{m}\right) \mid A^{n}\right)} \geq & 1+\inf _{U^{m}} \Xi_{\delta}\left(U^{m} \underset{U \backslash V^{m}}{\overleftarrow{L}} U \backslash V^{m}\right) \\
& +\inf _{U^{m}} \Xi_{\delta}\left(U^{m} \underset{U \backslash V^{m}}{\overleftarrow{ }} A^{m} \leftarrow U\right) .
\end{aligned}
$$

By (4.44) and (4.46),

$$
\begin{gathered}
\inf _{U^{m}} \Xi_{\delta}\left(U^{m} \overleftarrow{U \backslash V^{m}} U \backslash V^{m}\right) \geq C^{-1} \tau_{m}^{2-\delta} \frac{\eta_{m}}{\rho_{m}}, \\
\inf _{U^{m}} \Xi_{\delta}\left(U^{m} \overleftarrow{U \backslash V^{m}} A^{m}\right) \geq C^{-1} .
\end{gathered}
$$

By bounded oscillation of the Poincaré series in $A^{m}$ and (4.59), we have

$$
\inf _{U^{m}} \Xi_{\delta}\left(U^{m} \underset{U \backslash V^{m}}{\longleftarrow} A^{m} \leftarrow U\right) \geq C^{-1} \omega_{m}(\delta) .
$$

Plugging (4.58) and (4.60) in (4.57), and using (4.43) one gets (4.13).

\section{TRICHOTOMY}

Let $f$ be a periodic point of renormalization of period $p$. In this case, we may assume that the fundamental annulus $A^{n}$ forms a periodic sequence up to rescaling by $\rho_{n}^{-1 / 2}$ (see Remark A.2). Then $\eta_{m, n}, \xi_{m, n}, \ldots$ only depend on $n-m$ and the congruence class of $m$ modulo $p$.

The results in this section will be obtained from some particular cases of the estimates in Lemmas 4.2 and 4.4. Setting $n=2 m$ an integer multiple of $p$, the 
estimates can be rewritten as

$$
\begin{gathered}
\omega_{m}(\delta) \geq C^{-1} \tau_{m}^{2-\delta} \frac{\eta_{m}}{\rho_{m}}+\max \left\{1-C\left(\eta_{m}+\xi_{m}\right), 0\right\} \omega_{m}(\delta)+C^{-1} v_{m}^{2-\delta} \xi_{m} \rho_{m} \omega_{2 m}(\delta), \\
\omega_{2 m}(\delta) \geq C^{-1}\left(\tau_{m}^{2-\delta} \frac{\eta_{m}}{\rho_{m}}+\omega_{m}(\delta)\right) \omega_{m}(\delta) .
\end{gathered}
$$

Putting the respective estimates together, we get the following quadratic estimates:

$$
\omega_{m}^{[j+1]}(\delta) \leq C \frac{\eta_{m}}{\rho_{m}}+\left(1-C^{-1}\left(\eta_{m}+\xi_{m}\right)+C \eta_{m} \xi_{m}\right) \omega_{m}^{[j]}(\delta)+C \xi_{m} \rho_{m} \omega_{m}^{[j]}(\delta)^{2}, \quad \delta \approx 2,
$$

$$
\begin{aligned}
\omega_{m}(\delta) \geq & C^{-1} \tau_{m}^{2-\delta} \frac{\eta_{m}}{\rho_{m}}+\left(\max \left\{1-C\left(\eta_{m}+\xi_{m}\right), 0\right\}+C^{-1}\left(\tau_{m} v_{m}\right)^{2-\delta} \eta_{m} \xi_{m}\right) \omega_{m}(\delta) \\
& +C v_{m}^{2-\delta} \xi_{m} \rho_{m} \omega_{m}(\delta)^{2}
\end{aligned}
$$

Let us now explore those relations.

Lemma 5.1. Let $f$ be a periodic point of renormalization of period $p$. If $m=$ $0 \bmod p$, then there exists $C>0$ (only depending on the geometric bounds) such that

(1) If the polynomial

$$
P: x \mapsto C \frac{\eta_{m}}{\rho_{m}}+\left(1-C^{-1}\left(\eta_{m}+\xi_{m}\right)+C \eta_{m} \xi_{m}\right) x+C \xi_{m} \rho_{m} x^{2}
$$

has a real positive fixed point, then $\delta_{\mathrm{cr}}<2$ and for $\delta$ close to 2 , the smallest such fixed point gives an upper bound on $\omega_{m}(\delta)$.

(2) If $\delta \geq \delta_{\mathrm{cr}}$, the polynomial

$$
\begin{aligned}
Q_{\delta}: x & \mapsto C^{-1} \tau_{m}^{2-\delta} \frac{\eta_{m}}{\rho_{m}}+\left(\max \left\{1-C\left(\eta_{m}+\xi_{m}\right), 0\right\}+C^{-1}\left(\tau_{m} v_{m}\right)^{2-\delta} \eta_{m} \xi_{m}\right) x \\
& +C v_{m}^{2-\delta} \xi_{m} \rho_{m} x^{2}
\end{aligned}
$$

has a fixed point, and the smallest (respectively, largest) such fixed point gives a lower bound (respectively, upper bound) to $\omega_{m}(\delta)$.

Proof. By repeated application of (5.5), for $\delta \approx 2$ and for every $j \geq 0$, we have $\omega_{m}^{[j]}(\delta) \leq P^{j+1}(0)$. If there exists a positive fixed point for $P$, then the smallest such fixed point attracts the orbit of 0 . This gives the first statement.

By (5.6), for $\delta>\delta_{\mathrm{cr}}, Q_{\delta}\left(\omega_{m}(\delta)\right) \leq \omega_{m}(\delta)$. Thus $\omega_{m}(\delta)$ is contained in the interval bounded by the fixed points of $Q_{\delta}$. This still holds for $\delta=\delta_{\text {cr }}$ by continuity of the coefficients of $Q_{\delta}$. This implies the second statement. 
Theorem 5.2 (Balanced case). Let $f$ be a periodic point of renormalization of period $p$. Then there exists $C>0$, only depending on the geometric bounds, such that if $\delta_{\mathrm{cr}}=2$, then

$$
C^{-1} \leq \frac{\eta_{m}}{\xi_{m}} \leq C, \quad m \equiv 0 \bmod p
$$

Moreover,

$$
\xi_{m} \asymp \eta_{m} \asymp \frac{1}{m}
$$

for all $m \in \mathbb{N}$ with the constants also depending on $p$.

Proof. If (5.9) does not hold, then

$$
\xi_{m} \eta_{m}<<\left(\xi_{m}+\eta_{m}\right)^{2},
$$

and then the quadratic polynomial (5.7) has two positive fixed points. By the first part of Lemma 5.1, $\delta_{\text {cr }}<2$, contradicting the assumption. The same argument applied to the $k$-fold renormalization of $f, k=0,1, \ldots, p-1$, gives

$$
\eta_{m} \asymp \xi_{m}, \quad m \equiv k \bmod p,
$$

with the constant depending on $k$. Thus, $\eta_{m} \asymp \xi_{m}$ for all $m$, with the constant depending on $p$. By Lemma 4.1, this implies that

$$
\eta_{m}-C \eta_{m}^{2} \leq \eta_{m+1} \leq \eta_{m}-C^{-1} \eta_{m}^{2} .
$$

Hence

and thus $1 / \eta_{m} \asymp m$.

$$
C^{-1} \leq \frac{1}{\eta_{m+1}}-\frac{1}{\eta_{m}} \leq C
$$

The following result gives Theorem D stated in the Introduction.

Corollary 5.3. Let $f$ be a periodic point of renormalization. If $|J(f)|>0$, then $\delta_{\text {cr }}<2$.

Proof. Since $f$ is a renormalization periodic point, $f_{m}: U^{m} \rightarrow V^{m}$ is a rescaling of $f: U \rightarrow V$ for $m \equiv 0 \bmod p$. Hence

$$
\eta_{m}=p\left(X_{m} \mid U^{m}\right) \geq p\left(J\left(f_{m}\right) \mid U^{m}\right)=p(J(f) \mid U)>0, \quad m \equiv 0 \bmod p .
$$

Then by Lemma 4.1, $\xi_{m} \rightarrow 0$, so that (5.9) is violated; this contradicts $\delta_{\mathrm{cr}}=2$.

Lemma 5.4. Let $f$ be a periodic point of renormalization of period $p$ and let $m \equiv$ $0 \bmod p$. Then there exists $C>0$ depending only on the geometric bounds such that

$$
\begin{gathered}
\omega_{m}(2) \geq C^{-1} \frac{\eta_{m}}{\left(\xi_{m}+\eta_{m}\right) \rho_{m}} \quad \text { if } \quad \eta_{m} \xi_{m}<C^{-1}, 11 \\
\omega_{m}(2) \leq C \frac{\eta_{m}}{\left(\xi_{m}+\eta_{m}\right) \rho_{m}} \quad \text { if } \quad\left|\ln \frac{\eta_{m}}{\xi_{m}}\right|>C, \\
\omega_{2 m}(2) \asymp\left(\frac{\eta_{m}}{\rho_{m}}+\omega_{m}(2)\right) \omega_{m}(2) \asymp \omega_{m}(2)^{2} .
\end{gathered}
$$

In particular, if $\eta_{m} \xi_{m}<C^{-1}$ and if either $\eta_{m} / \xi_{m}$ or $\omega_{m} \rho_{m}$ is small, then both are small and indeed they are comparable.

\footnotetext{
${ }^{11}$ Note that this assumption is always satisfied on high levels since $\eta_{m} \xi_{m} \rightarrow 0$ by Lemma 4.1.
} 
Proof. If $\eta_{m} \xi_{m}$ is small, then the linear coefficient of $Q_{2}$ is comparable with $\eta_{m}+\xi_{m}$, and a direct calculation of the smallest fixed point of $Q_{2}$ gives the lower bound (5.12) on $\omega_{m}(2)$.

If $\left|\ln \left(\eta_{m} / \xi_{m}\right)\right|$ is large, then (5.11) holds, and the polynomial $P$ has two real fixed points. A direct calculation of the smallest one gives the upper bound (5.13) on $\omega_{m}(2)$.

By (5.2), (5.4),

$$
\omega_{2 m}(2) \asymp\left(\frac{\eta_{m}}{\rho_{m}}+\omega_{m}(2)\right) \omega_{m}(2) .
$$

By $(5.3), \omega_{m}(2)>C^{-1} \eta_{m} / \rho_{m}$, which shows that $\left(\eta_{m} / \rho_{m}\right)+\omega_{m}(2) \asymp \omega_{m}(2)$, which together with (5.15) implies (5.14).

The last assertion is a straightforward consequence of (5.12) and (5.13).

Theorem 5.5 (Lean case). Let $f$ be a periodic point of renormalization of period $p$. Then there exists $C>0$, only depending on the geometric bounds, such that if for some $m \equiv 0 \bmod p$ we have

$$
\eta_{m}<C^{-1} \xi_{m},
$$

then inf $\xi_{n}>0 .{ }^{12}$ Moreover, $\eta_{n} \rightarrow 0$ exponentially fast.

Proof. Since $\eta_{n}$ monotonically decrease, assumption (5.16) implies that $\eta_{n}$ (and hence $\eta_{n} \xi_{n}$ ) are small for $n \geq m$. It also implies by Lemma 5.4 that $\omega_{m} \rho_{m}$ is small.

By $(5.14), \omega_{2 m} \leq K \omega_{m}^{2}$, so that $K \omega_{2 m} \rho_{2 m} \leq\left(K \omega_{m} \rho_{m}\right)^{2}$. Iterating this estimate $k$ times, we conclude that

$$
K \omega_{2^{k} m} \rho_{2^{k} m} \leq\left(K \omega_{m} \rho_{m}\right)^{2^{k}} \equiv q^{2^{k}}
$$

where $q<1$ provided $\omega_{m} \rho_{m}$ is small enough. By Lemma 5.4,

$$
\frac{\eta_{2^{k} m}}{\xi_{2^{k} m}}=O\left(q^{2^{k}}\right)
$$

Since $\eta_{n}$ monotonically decrease, we conclude that they decay exponentially: $\eta_{n}=$ $O\left(q^{n / 2}\right)$.

Furthermore, it follows from the renormalization periodicity that the $\xi_{k p}$ monotonically decrease in $k$. It is also easy to check that $\xi_{n+1} \asymp \xi_{n}$. It follows that

$$
\inf \xi_{n}=0 \Longrightarrow \xi_{n} \rightarrow 0 \text {. }
$$

Since $\eta_{n}$ decay exponentially, $\lim \sup \xi_{n}>0$ by Lemma 4.1 . Hence $\xi_{n}$ are bounded away from 0 .

Lemma 5.6. Let $f$ be a periodic point of renormalization of period $p$ and let $m \equiv$ $0 \bmod p . \quad$ If area $(J(f))=0$, then there exists $C>0$, only depending on the geometric bounds, such that

$$
C^{-1} \frac{\eta_{m}}{\xi_{m} \rho_{m}} \leq \omega_{m}(2) \leq C \frac{\eta_{m}}{\xi_{m} \rho_{m}} .
$$

\footnotetext{
${ }^{12}$ An explicit lower bound for inf $\xi_{n}$ in terms of the geometric and combinatorial bounds and the period of $f$ can be worked out.
} 
Proof. Since the set $Y_{m}$ is wandering,

$$
\int_{Y_{m}} \Xi_{2}(f, x) d x=\operatorname{area}\left(\bigcup_{n=0}^{\infty} f^{-n}\left(Y_{m}\right)\right) \equiv\left|Z_{m}\right| .
$$

The set $Z_{m}$ consists of points that land at $A^{m}$ at least once but at most finitely many times. Hence

$$
X_{m} \backslash J(f) \subset Z_{m} \subset X_{m}
$$

Since area of the Julia set is zero, $\left|Z_{m}\right|=\left|X_{m}\right|$ and hence

$$
\int_{Y_{m}} \Xi_{2}(f, x) d x=\left|X_{m}\right| \text {. }
$$

By bounded oscillation of the Poincaré series,

$$
\omega_{m}(2) \asymp \frac{1}{\left|Y_{m}\right|} \int_{Y_{m}} \Xi_{2}(f, x) d x=\frac{\left|X_{m}\right|}{\left|Y_{m}\right|} \asymp \frac{\eta_{m}}{\xi_{m} \rho_{m}} .
$$

Lemma 5.7. Let $f$ be a periodic point of renormalization of period $p$. If $\delta_{\mathrm{cr}}<2$, then there exists $\theta<1$, only depending on $\delta_{\mathrm{cr}}$ and the geometric bounds, such that

$$
\frac{\eta_{m} \xi_{m}}{\left(\eta_{m}+\xi_{m}\right)^{2}} \leq \theta^{m}, \quad m \equiv 0 \bmod p .
$$

Proof. By Lemma 5.1, the quadratic polynomial $Q_{\delta_{\mathrm{cr}}}$ has a real fixed point and hence has the positive discriminant. Together with Lemma 4.3 this implies (5.20).

Theorem 5.8 (Black hole case). Let $f$ be a periodic point of renormalization of period $p$. Then there exists $C>0$, only depending on the geometric bounds, such that if for some $m \equiv 0 \bmod p$ we have

$$
\eta_{m}>C \xi_{m}
$$

then $\lim \eta_{n}>0 .^{13}$ Moreover, $\xi_{n} \rightarrow 0$ exponentially fast.

Proof. Assumption (5.21) allows us to apply (5.13). Since (5.21) and (5.13) are not compatible with (5.17), area of the Julia set must be positive and hence $\lim \eta_{n}>0 .{ }^{14}$

Moreover, by Corollary 5.3, $\delta_{\text {cr }}<2$, which implies by Lemma 5.7 that $\eta_{n} \xi_{n} \rightarrow 0$ exponentially fast, so $\xi_{n} \rightarrow 0$ exponentially fast as well.

\section{Conservativity}

In order to complete the proof of Theorem $\mathrm{E}$ we must show that some Feigenbaum maps have a conservative $\delta_{\text {cr }}$ conformal measure. In this section we will provide a criterium for conservativity, and in the next section we will show that such criterium is satisfied by some Feigenbaum maps

Theorem 6.1. Let $f$ be a Feigenbaum map. ${ }^{15}$ If $\delta_{\mathrm{cr}}(f)>\delta_{\mathrm{cr}}\left(R^{n}(f)\right)$, then the $\delta_{\mathrm{cr}}(f)$ conformal measure on $J(f)$ is conservative.

\footnotetext{
${ }^{13}$ A lower bound for $\lim \eta_{n}$ in terms of the geometric and combinatorial bounds and the period of $f$ can be worked out.

${ }^{14}$ Notice that this conclusion gives a criterion for positive measure which might be checkable numerically. Let us remark that this conclusion is based on an upper bound on $\omega_{m}(2)$, which is derived from Lemma 4.2 .

${ }^{15} \mathrm{It}$ is enough to assume that $f$ is $n$ times renormalizable.
} 
Proof. Let $\delta_{\mathrm{cr}} \equiv \delta_{\mathrm{cr}}(f)$ and let $\mu$ be the $\delta_{\text {cr }}$ conformal measure on $J(f)$. Let $Q \subset A^{n}$ be the set of points that return to $A^{n}$. By Lemma $3.1, \mu$ is conservative if and only if $\mu(Q)=\mu\left(A^{n}\right)$. We will show that if $\mu(Q)<\mu\left(A^{n}\right)$, then

$$
E_{\mu}\left(\Xi_{\delta}^{[j+1]}\left(A^{n} \leftarrow U\right) \mid A^{n}\right) \leq C+\kappa E_{\mu}\left(\Xi_{\delta}^{[j]}\left(A^{n} \leftarrow U\right) \mid A^{n}\right), \quad \delta \approx \delta_{\mathrm{cr}},
$$

for some $C>0, \kappa<1$. This implies that $E_{\mu}\left(\Xi_{\delta}\left(A^{n} \leftarrow U\right) \mid A^{n}\right) \leq C /(1-\kappa)$ for $\delta \approx \delta_{\mathrm{cr}}$, which contradicts the definition of $\delta_{\mathrm{cr}}$ and concludes the proof.

Through the argument we will skip the notation for truncation. We start with the estimate

$$
\begin{aligned}
E_{\mu}\left(\Xi_{\delta}\left(A^{n} \leftarrow U\right) \mid A^{n}\right)= & 1+E_{\mu}\left(\Xi_{\delta}\left(A^{n} \overleftarrow{U \backslash A^{n}} U \backslash A^{n}\right) \mid A^{n}\right) \\
& +E_{\mu}\left(\Xi_{\delta}\left(A^{n} \overleftarrow{U \backslash A^{n}} A^{n} \leftarrow U\right) \mid A^{n}\right) .
\end{aligned}
$$

Let

$$
\nu_{\delta}=\int_{A^{n}} \sum_{\left(x_{0}, \ldots, x_{k}=x\right) \in \mathcal{F}} \frac{\delta_{x_{0}}}{\left|D f^{k}\left(x_{0}\right)\right|^{\delta}} d \mu(x), \quad \mathcal{F}=\left(A^{n} \stackrel{+}{U \backslash A^{n}} A^{n}\right)
$$

Then $\nu_{\delta_{\mathrm{cr}}}=\mu \mid Q$. Notice that

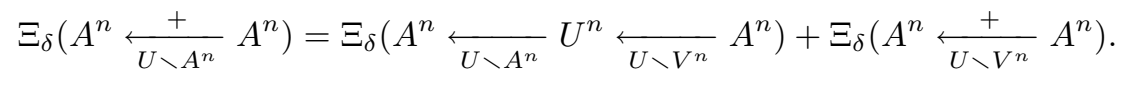

The assumption of dissipativity of $\mu$ implies in particular that

$$
\sup \Xi_{\delta_{\mathrm{cr}}}\left(V^{n} \underset{U \backslash V^{n}}{+} A^{n}\right)<\infty
$$

By hyperbolicity of the set of orbits avoiding $V^{n}$, and by Lemma 2.12, we have that $\Xi_{\delta}\left(V^{n} \underset{U \backslash V^{n}}{+} A^{n}\right)$ is uniformly bounded in a neighborhood of $\delta_{\mathrm{cr}}$. Notice that

$$
\Xi_{\delta}\left(A^{n} \underset{U \backslash A^{n}}{\longleftarrow} U^{n}\right)=\Xi_{\delta}\left(A^{n} \stackrel{f_{n}}{\longleftarrow} U^{n}\right),
$$

which by hypothesis is uniformly bounded in a neighborhood of $\delta_{\mathrm{cr}}$. Thus

$$
\Xi_{\delta}\left(A^{n} \underset{U \backslash A^{n}}{+} A^{n}\right)<C, \quad \delta \approx \delta_{\mathrm{cr}}
$$

and it follows immediately (see Lemma 4.5) that $\delta \mapsto \nu_{\delta}(N)$ is continuous in a neighborhood of $\delta_{\text {cr }}$ for any Borelian set $N$. If $\mu(Q)<\mu\left(A^{n}\right)$ it then follows that (6.7)

$$
\frac{E_{\mu}\left(\Xi_{\delta}\left(A^{n} \stackrel{+}{U \backslash A^{n}} A^{n} \leftarrow U\right) \mid A^{n}\right)}{E_{\mu}\left(\Xi_{\delta}\left(A^{n} \leftarrow U\right) \mid A^{n}\right)}=\frac{E_{\nu_{\delta}}\left(\Xi_{\delta}(Q \leftarrow U) \mid A^{n}\right)}{E_{\mu}\left(\Xi_{\delta}\left(A^{n} \leftarrow U\right) \mid A^{n}\right)} \leq \kappa<1, \quad \delta \approx \delta_{\mathrm{cr}} .
$$

Similarly to (6.6) we conclude that

$$
\begin{aligned}
E_{\mu}\left(\Xi _ { \delta } \left(A^{n} \overleftarrow{U \backslash A^{n}}\right.\right. & \left.\left.U \backslash A^{n}\right) \mid A^{n}\right)=E_{\mu}\left(\Xi_{\delta}\left(A^{n} \overleftarrow{U \backslash A^{n}} U^{n}\right) \mid A^{n}\right) \\
& +E_{\mu}\left(\Xi_{\delta}\left(A^{n} \overleftarrow{U \backslash V^{n}} U \backslash V^{n}\right) \mid A^{n}\right) \\
& +E_{\mu}\left(\Xi_{\delta}\left(A^{n} \overleftarrow{\longleftarrow \backslash A^{n}} U^{n} \overleftarrow{U \backslash V^{n}} U \backslash V^{n}\right) \mid A^{n}\right)<C, \quad \delta \approx \delta_{\mathrm{cr}} .
\end{aligned}
$$

Plugging (6.8) and (6.7) into (6.2) we get (6.1). 


\section{VARYING THE DIMENSION}

In this section we will give two proofs that for some Feigenbaum maps, $\delta_{\mathrm{cr}}(R f)<$ $\delta_{\mathrm{cr}}(f)$. Together with Theorem 6.1, it will justify the assertion of Theorem E that the $\delta_{\mathrm{cr}}$-conformal measure can be conservative (up to the proof of the existence of Lean Feigenbaum Julia sets, which will be completed in $\S 9)$.

7.1. Varying the dimension within the horseshoe. Below we will make use of the continuity properties of the critical exponent (Corollary 3.15 and Lemma 3.16) and an observation that it is non-increasing under the renormalization:

$$
\delta_{\mathrm{cr}}(R f) \leq \delta_{\mathrm{cr}}(f)
$$

Lemma 7.1. Let $f$ be a Feigenbaum map. If $\delta_{\mathrm{cr}}(f)=\delta_{\mathrm{cr}}(R(f))$, then $\delta_{\mathrm{cr}}(f)=$ $\delta_{\mathrm{cr}}\left(R^{n}(f)\right)$ for all $n \geq 0$.

Proof. Assume that $\delta_{\mathrm{cr}}(f)=\delta_{\mathrm{cr}}(R(f))>\delta_{\mathrm{cr}}\left(R^{2}(f)\right)$. Then the $\delta_{\mathrm{cr}}(R(f))$ conformal measure on $J(R(f))$ is dissipative by the first relation (Proposition 3.6) and conservative by the second one (Theorem 6.1), a contradiction.

Let us consider the horseshoe $\mathcal{A} \equiv \mathcal{A}_{\mathcal{M}}$ associated to some fine collection $\mathcal{M}$ of little Mandelbrot copies (see $\S 2.5$ ). Notice that under the renormalization operator $R: \mathcal{A} \rightarrow \mathcal{A}$ the orbit of a generic point $f \in \mathcal{A}$ is dense in $\mathcal{A}$.

Lemma 7.2. Assume that $\operatorname{orb}_{R}(f)$ is dense in $\mathcal{A}$. Then

$$
\delta_{\mathrm{cr}}(f)=\max _{g \in \mathcal{A}} \delta_{\mathrm{cr}}(g) .
$$

Proof. This follows from the lower semi-continuity of the critical exponent and (7.1).

Lemma 7.3. If the critical exponent is not constant over a renormalization horseshoe $\mathcal{A}$ (associated with some fine collection of Mandelbrot copies), then there exists $f \in \mathcal{A}$ such that $\delta_{\mathrm{cr}}(f)>\delta_{\mathrm{cr}}(R f)$.

Proof. Take two maps $f_{+}, f_{-} \in \mathcal{A}$ such that $\delta_{\mathrm{cr}}\left(f_{+}\right)>\delta_{\mathrm{cr}}\left(f_{-}\right)$. Since the critical exponent is lower semi-continuous, $f_{+}$can be selected to be a periodic point of some period $p$ of $R$. Let $f$ belong to the intersection of the unstable manifold of $f_{+}$with the stable manifold of $f_{-}$. Using semi-continuity again, we conclude that

$$
\lim \delta_{\mathrm{cr}}\left(R^{-n p}(f)\right) \geq \delta_{\mathrm{cr}}\left(f_{+}\right) .
$$

On the other hand, by monotonicity (7.1) and uniform continuity on hybrid classes (Lemma 3.16),

$$
\lim \delta_{\mathrm{cr}}\left(R^{n} f\right)=\lim \delta_{\mathrm{cr}}\left(R^{n} f_{-}\right) \leq \delta_{\mathrm{cr}}\left(f_{-}\right) .
$$

Example 7.1. Let $f_{p}$ be the real fixed point of renormalization of period $p$ with combinatorics closest to Chebyshev. By Lemma 2.5, $\Xi_{1}\left(f_{p}, z\right)=\infty$. On the other

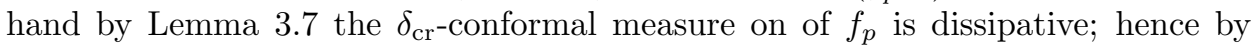
Lemma $3.1, \Xi_{\delta_{\text {cr }}}\left(f_{p}, z\right)<\infty$. Hence $\delta_{\text {cr }}\left(f_{p}\right)>1 .{ }^{16}$

Moreover, it is shown in [AL] that $\lim \delta_{\mathrm{cr}}\left(f_{p}\right)=1$. Thus we can choose $p_{1}$ and $p_{2}$ such that $\delta_{\mathrm{cr}}\left(f_{p_{1}}\right)>\delta_{\mathrm{cr}}\left(f_{p_{2}}\right)$, so the horseshoe associated to the corresponding Mandelbrot copies satisfies the hypothesis of the previous lemma.

\footnotetext{
${ }^{16}$ This also follows, from Theorem 2.4 and Corollary 3.13, in the case of $p$ large, which we use here (since in this case the Julia set has zero Lebesgue measure).
} 
7.2. Varying the dimension within a hybrid class. Recall that $\mathcal{H}_{f}$ stands for the hybrid class of a quadratic-like map $f$ (see $\S 2.3$ ). We will now study how the dimension $g \mapsto \operatorname{HD}(J(g))$ varies over $\mathcal{H}_{f}$.

Lemma 7.4. Let $f$ be a Feigenbaum map. Then

$$
\sup _{g \in \mathcal{H}_{f}} \operatorname{HD}(J(g))=2 .
$$

Proof. Let us take a little square $\Delta \Subset \mathbb{V} \backslash \mathcal{O}$ intersecting the Julia set. It is easy to find four branches $g_{i} \equiv f_{i}^{-n_{i}} \mid \Delta$ of inverse iterates such that $D_{i} \equiv g_{i}(\Delta) \Subset \Delta$ and $f^{n_{i}}$ is the first return map from $D_{i}$ to $\Delta$. Let $S=\bigcup D_{i}$ and let $G: S \rightarrow \Delta$ be the associated Bernoulli map, $G \mid D_{i}=f^{n_{i}}$. Let $Q$ be the maximal $G$-invariant set contained in $S$. By the Schwarz Lemma, $Q$ is hyperbolic for $G$, so that the forward saturation $\operatorname{orb}(Q)$ is a hyperbolic set for $f$.

Let us consider a diffeomorphism $H: \mathbb{C} \rightarrow \mathbb{C}$ such that $\tilde{\Delta} \equiv h(\Delta)$ is the standard square $[0,1] \times[0,1]$ of size 1 and $\tilde{D}_{i} \equiv H\left(D_{i}\right)$ are rescaled standard squares of size close to $1 / 4$ (and we can select $H$ to be affine on $\mathbb{C} \backslash \Delta$ ). Let $\tilde{S}=\bigcup \tilde{D}_{i}$. Consider a piecewise affine $\tilde{G}: \tilde{S} \rightarrow \tilde{\Delta}$ that affinely maps each $\tilde{D}_{i}$ onto $\tilde{\Delta}$. Then the maximal invariant set $\tilde{Q}$ of $\tilde{G}$ has Hausdorff dimension $2-\epsilon$.

By the standard pullback argument, the maps $G: S \rightarrow \Delta$ and $\tilde{G}: \tilde{S} \rightarrow \tilde{\Delta}$ are conjugate by some qc homeomorphism $h$ which is affine on $\mathbb{C} \backslash \Delta$. Let us consider the corresponding $G$-invariant Beltrami differential $\mu=\bar{\partial} h / \partial h$.

Let $f: \mathbb{U} \rightarrow \mathbb{V}$ and let $W^{m}=f^{-m} \mathbb{U}$. For $m \geq 0$, define a Beltrami differential $\mu_{m}$ as follows. Let $\mu_{m}=\mu$ on $\mathbb{C} \backslash W^{m}$. Pull it back by the iterates of $f$ to the domain $W^{m} \backslash J(f)$. Finally, let $\mu_{m} \mid J(f)=0$. Since $G$ is composed from iterates of $f$, this Beltrami differential is simultaneously invariant with respect to $f: W^{m} \rightarrow W^{m-1}$ and $G: S \rightarrow \Delta$. Since conformal pullbacks preserve the norm of Beltrami differentials, $\left\|\mu_{m}\right\|_{\infty} \leq\|\mu\|_{\infty}$.

We now assume that area $(J(f))=0$, for otherwise there is nothing to prove. In this case $\mu_{m} \rightarrow \mu$ almost everywhere.

Let $h_{m}$ be the solution of the Beltrami equation $\bar{\partial} h_{m} / \partial h_{m}=\mu_{m}$ coinciding with $h$ at three points. Then $h_{m} \rightarrow h$ uniformly on $\overline{\mathbb{C}}$.

Consider a quadratic-like map

$$
g_{m}=h_{m} \circ f \circ h_{m}^{-1}: h_{m}\left(W^{m}\right) \rightarrow h_{m}\left(W^{m-1}\right)
$$

and the corresponding expanding map

$$
G_{m}=h_{m} \circ G \circ h_{m}^{-1}: h_{m}(S) \rightarrow h_{m}(\Delta) .
$$

Since the latter maps uniformly converge to $\tilde{G}, \operatorname{HD}\left(h_{m}(Q)\right) \rightarrow \operatorname{HD}(\tilde{Q})=2-\epsilon$. The conclusion follows since the forward saturation of $h_{m}(Q)$ under $g_{m}$ is a hyperbolic subset of $J\left(g_{m}\right)$.

Remark 7.1. In case of the Julia set of positive area, it would be interesting to obtain the same result for the hyperbolic dimension $\operatorname{HD}_{\text {hyp }}(g)$ rather than $\operatorname{HD}(J(g))$.

Let us consider a fine family $\mathcal{M}$ of little Mandelbrot copies and a Feigenbaum map $f$ with combinatorics $\bar{M}_{+}=\left\{M_{0}, M_{1}, \ldots\right\}, M_{i} \in \mathcal{M}$. We say that $f$ has recurrent combinatorics if the sequence $\bar{M}_{+}$is recurrent. Such a sequence can be extended to the "past" as a bi-infinite recurrent sequence $\bar{M}=\left\{M_{k}\right\}_{k \in Z}$. Take the map $f_{*}$ in the renormalization horseshoe $\mathcal{A}_{\mathcal{M}}$ with combinatorics $\bar{M}$. It is 
recurrent under the renormalization dynamics, and the $R$-orbits of $f$ and $f_{*}$ are forward asymptotic. We say that $f_{*}$ is "associated" with $f$.

Theorem 7.5. Let $f$ be a Feigenbaum map with recurrent combinatorics, and let $f_{*}$ be an associated map in the horseshoe $\mathcal{A}_{\mathcal{M}}$. Then the function $h: g \mapsto \operatorname{HD}(J(g))$, $g \in \mathcal{H}_{f}$, is either identically equal to 2 , or

$$
\operatorname{Im} h=\left[\operatorname{HD}\left(J\left(f_{*}\right)\right), 2\right) .
$$

Proof. Since qc maps preserve the property $\operatorname{HD}(J)=2$, the function $h$ is either identically equal to 2, or always less than 2. Assume that the latter happens.

Since hybrid classes are connected and the Hausdorff dimension varies continuously under qc deformations, $\operatorname{Im}(h)$ is an interval. By Lemma 7.4, it must be either $[a, 2)$ or $(a, 2)$.

Since the function $h$ is a decaying Lyapunov function for the renormalization (i.e., $h(R g) \leq h(g)$ ), and by the recurrence assumption the orbit $R^{n} f$ accumulates on $f_{*}$, we conclude that $h(f) \geq h\left(f_{*}\right)$. Hence

$$
h\left(f_{*}\right)=\min _{g \in \mathcal{H}_{f}} h(g),
$$

and we are done.

Let us take, for instance, a renormalization fixed point $f_{*}$ of lean type. Then $\operatorname{HD}\left(J\left(f_{*}\right)\right)<2$ and there is a map $f \in \mathcal{H}_{f_{*}}$ with $\operatorname{HD}(J(f))>\operatorname{HD}\left(J\left(f_{*}\right)\right)$. Since $R^{n} f \rightarrow f_{*}$ and the critical exponent is continuous on hybrid classes, there is an $n$

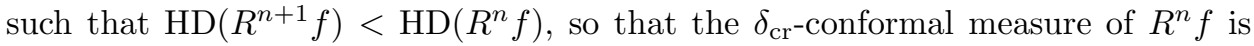
conservative.

\section{INTERPOLATION ARGUMENT}

Let us now prove Theorem B.

Theorem 8.1. Let $\mathcal{A}$ be the horseshoe associated to some fine collection of Mandelbrot copies. If there exist $g_{+}, g_{0} \in \mathcal{A}$ such that area $\left(J\left(g_{+}\right)\right)>0$ and area $\left(J\left(g_{0}\right)\right)=0$, then for a generic $f \in \mathcal{A}, \delta_{\mathrm{cr}}(f)=2$ and area $(J(f))=0$.

Proof. Any map in the stable manifold of $g_{0}$ has a Julia set of zero area. Since the stable manifold of $g_{0}$ is dense in $\mathcal{A}$ and area $J(g)$ depends upper semi-continuously on $g \in \mathcal{A}$, it follows that a generic map in $\mathcal{A}$ has a Julia set of zero area.

Fix $s>0$ and let $f_{[s]} \in \mathcal{A}$ be such that $R^{s}\left(f_{[s]}\right)$ belongs to the intersection of the stable manifold of $g_{0}$ and the local unstable manifold of $R^{s}\left(g_{+}\right)$, so that the combinatorics of $f_{[s]}$ coincides with that of $g_{+}$on many top renormalization levels and coincides with that of $g_{0}$ on all sufficiently deep renormalization levels. Let us denote by $\phi_{n, s}$ the first "univalent" return map to the disk $V^{n, s} \equiv V^{n}\left(f_{[s]}\right)$ (see $\S 2.7)$, that is, $\phi_{n, s}(z)=f_{[s]}^{k}(z)$ if $k$ is smallest possible such that there is a univalent branch of $f_{[s]}^{-k} \mid V^{n, s}$ onto a neighborhood of $z$. Since the maps $\phi_{n, s}$ are Markov and have bounded distortion (see Remark 2.1), the critical exponent $\delta_{\mathrm{cr}}\left(\phi_{n, s}\right)$ makes sense (i.e., it is independent of the base point $z \in V^{n, s}$ in the Poincaré series $\left.\Xi_{\delta}\left(\phi_{n, s}, z\right)\right)$. Obviously $\delta_{\mathrm{cr}}\left(f_{[s]}\right) \geq \delta_{\mathrm{cr}}\left(\phi_{n, s}\right)$. Let us show that we can choose $n, s$ so that $\delta_{\mathrm{cr}}\left(\phi_{n, s}\right)$ is close to 2 , as this will imply the result by Lemma 7.2.

Let $D_{k}^{n, s}$ be the domain of $\phi_{n, s}^{k}$. We may assume that $\left|D \phi_{n, s}(x)\right|>2$ for $x \in$ $D_{1}^{n, s}$; see Remark A.1. 
Let us show that we can choose $n$ and $s$ so that $p\left(D_{1}^{n, s} \mid V^{n, s}\right)$ is close to 1 . Since $J\left(g_{+}\right)$has positive Lebesgue measure, its critical point is a density point of $J\left(g_{+}\right)$. Given $\xi>0$, we can choose $\rho>0$ so that $p\left(J\left(g_{+}\right) \mid \mathbb{D}_{\rho}\right)>1-\xi$. Take the minimal $n>0$ such that $\operatorname{diam}\left(V^{n, s}\right) \leq \rho / 2$ for all $s$ sufficiently big. Then for $s$ big, $\operatorname{diam}\left(V^{n, s}\right) \asymp \rho$, and so $V^{n, s} \supset \mathbb{D}_{q \rho}$ for some absolute $q>0$.

Let $A^{n, s}$ be defined in the natural way (see $\S 2.7$ ). Let $L^{s} \subset \mathbb{D}_{\rho}$ be the set of points whose positive orbit under $f_{s}$ intersects $\mathbb{D}_{q \rho}$. Notice that $L^{s} \cap A^{n, s} \subset D_{1}^{n, s} \cap A^{n, s}$. Since the positive orbit of almost every $x \in J\left(g_{+}\right)$(under iterates of $g_{+}$) accumulates on $0, \liminf L^{s} \supset J\left(g_{+}\right) \cap \mathbb{D}_{\rho}$ up to a set of zero area. Thus we can choose $s$ so that $p\left(L^{s} \mid \mathbb{D}_{\rho}\right)>1-\xi$. Hence $p\left(D_{1}^{n, s} \mid A^{n, s}\right)>1-C \xi$. By bounded distortion,

$$
p\left(D_{1}^{n, s} \mid B^{n, s}\right)>1-C \xi \quad \text { where } \quad B^{n, s}=\bigcup_{k \geq 0} f_{[s]}^{-k}\left(A^{n, s}\right) .
$$

Since $f_{[s]}$ is hybrid equivalent to $g_{0}, J\left(f_{[s]}\right)$ has zero area. Thus $B^{n, s}=V^{n, s}$ modulo a set of zero area, so that (8.1) is equivalent to $p\left(D_{1}^{n, s} \mid V^{n, s}\right)>1-C \xi$ as desired.

Fix $\epsilon>0$. Notice that if $p\left(D_{1}^{n, s} \mid V^{n, s}\right)$ is close to 1 , then $p\left(D_{k}^{n, s} \mid V^{n, s}\right)>(1-\epsilon)^{k}$. We have

$$
\begin{aligned}
\int_{V^{n, s}} \Xi_{2-\gamma}\left(\phi_{n, s}, x\right) d x & =\int_{V^{n, s}} \sum_{k \geq 0} \sum_{\phi_{n, s}^{k}(y)=x}\left|D \phi_{n, s}^{k}(y)\right|^{\gamma-2} d x \\
& \geq \int_{V^{n, s}} \sum_{k \geq 0} \sum_{\phi_{n, s}^{k}(y)=x}\left|D \phi_{n, s}^{k}(y)\right|^{-2} 2^{k \gamma} d x=\sum_{k \geq 0}\left|D_{k}^{n, s}\right| 2^{k \gamma} \\
& \geq\left|V^{n, s}\right| \sum_{k \geq 0} 2^{k \gamma}(1-\epsilon)^{k} .
\end{aligned}
$$

Thus the critical exponent $2-\gamma$ of $\phi_{n, s}$ satisfies $2^{\gamma}(1-\epsilon) \leq 1$, so by choosing $\epsilon$ small we can get $\delta_{\mathrm{cr}}\left(\phi_{n, s}\right)$ arbitrarily close to 2 .

Remark 8.1. Another interpolation argument allows one to show that, under the hypothesis of the previous theorem, there exists $f \in \mathcal{A}$ with a dense orbit (or which is periodic) and with area $J(f)>0$. Notice that if $f$ has a dense orbit, then it automatically has $\delta_{\mathrm{cr}}(f)=2$ by the previous theorem and Lemma 7.2, and if $f$ is periodic, then $\delta_{\mathrm{cr}}(f)<2$ (since we are in the lean case).

\section{LEAN CRITICAL POINTS}

In this section we will show that there are many Feigenbaum Julia sets with zero area (Yarrington's thesis $[Y]$ ). We will restrict ourselves to a particular class of combinatorics (to be defined below), for which the estimates are simpler. As a consequence of our estimates, we will also conclude that the lean case exists, completing the proof of the first assertion of Theorem A.

Let $F: \bigcup \Upsilon_{j} \rightarrow \Upsilon$ be a holomorphic map with the following properties:

(1) $\bigcup \Upsilon_{j} \subset \Upsilon$,

(2) for every $j \neq 0, F: \Upsilon_{j} \rightarrow \Upsilon$ is univalent,

(3) $f \equiv F: \Upsilon_{0} \rightarrow \Upsilon$ is a quadratic-like map with connected Julia set.

We let $\Upsilon^{k}=f^{-k}\left(\Upsilon_{0}\right)$.

We say that $F$ is $\kappa$-good if

(1) $\bmod \left(\Upsilon \backslash \bar{\Upsilon}_{0}\right) \geq \kappa$,

(2) the domain of the first return map to $\Upsilon_{0}$ has density at most $\kappa^{-1}$. 
Let $P$ be the (monic) quadratic polynomial which is hybrid equivalent to $f$ : $\Upsilon_{0} \rightarrow \Upsilon$. Denote by $D_{r}$ the domain bounded by the equipotential of height $r$ for $P$. We construct the standard Yoccoz puzzle of $P$ with the help of the equipotential at height 4 ; see for instance [L5]. Let $\hat{T}_{1} \supset \hat{T}_{2} \supset \ldots$ be the principal nest of $P$ obtained in the standard way (see [L5]).

We will make the following combinatorial assumptions:

(1) $P$ belongs to a selected finite family of Misiurewicz wakes, or, equivalently, $\hat{T}_{1}$ is mapped to the top level puzzle piece by an iterate $F^{n}$ with a bounded $n$ (see $[\mathrm{L} 6, \mathrm{Sc}])$;

(2) $P$ is renormalizable with no central cascades: there exists $\tau(P)>0$ (the height of $P$ ) such that the first return of 0 to $\hat{T}_{k}$ belongs to $\hat{T}_{k+1}$ if and only if $k \geq \tau(P)$.

In what follows, the constants involved will depend on the choice of the Misiurewicz wake.

We will need the following simple plane topology argument.

Lemma 9.1. Let $W_{i} \subset \mathbb{C}, i \geq 0$, be Jordan domains such that $\operatorname{diam} W_{j} \rightarrow 0$. Assume that $W_{0} \cap W_{i} \neq \emptyset, i>0$, while $\bar{W}_{i} \cap \bar{W}_{j}=\emptyset$ for $i>j>0$. Consider $a$ compact set $W \subset \mathbb{C}$ obtained by taking the closure of $\bigcup W_{i}$ and then filling in the holes. Then $W$ is a Jordan disk.

Proof. We may assume that $W_{0}$ is not contained in any $W_{i}$, for otherwise $W=W_{i}$. We can also discard the $W_{i}, i>0$, that are contained in $W_{0}$. It follows that $\#\left(\partial W_{i} \cap \partial W_{0}\right) \geq 2$ for $i \geq 0$.

Note that $\partial W \subset \bar{U} \partial W_{i}$. Moreover, if a sequence of domains $W_{i}$ accumulates on some $x \in \partial W$, then $x \in \partial W_{0}$. Hence $\partial W \subset \cup \partial W_{i}$.

It follows that each connected component of $\partial W \backslash \partial W_{0}$ is an open arc $\gamma_{k}$ contained in some $\partial W_{i}$, whose endpoints are contained in $\partial W_{0}$. (Warning: there can be infinitely many $\gamma_{k}$ 's in one $W_{i}$ ). Notice that the endpoints of $\gamma_{k}$ cannot coincide (otherwise it would bound some $W_{i}$ such that $\partial W_{i} \cap \partial W_{0}$ is a point), so $Q_{k} \equiv \bar{\gamma}_{k} \backslash \gamma_{k}$ consists of two points. Since diam $W_{i} \rightarrow 0$ we also have diam $\gamma_{k} \rightarrow 0$.

Let $\delta_{k}$ and $\delta_{k}^{\prime}$ denote the two components of $\partial W_{0} \backslash Q_{k}$. One of them, say, $\delta_{k}$, is such that $\gamma_{k} \cup Q_{k} \cup \delta_{k}$ bounds a domain contained in $W$ (since $W$ is a full compact set) which is disjoint from $W_{0}$. This shows that each $\delta_{k}$ is contained in int $W \cap \partial W_{0}$. Hence $\partial \delta_{k} \cap \delta_{i}=\emptyset$ for $k \neq i$, so that all the $\operatorname{arcs} \delta_{k}$ are disjoint. Since they lie on the Jordan curve $\partial W_{0}$, diam $\delta_{k} \rightarrow 0$.

Let $h_{k}: \delta_{k} \rightarrow \gamma_{k}$ be a homeomorphism fixing the endpoints, and let $h: \partial W_{0} \rightarrow \mathbb{C}$ be defined by $h \mid \delta_{k}=h_{k}$ and $h \mid \partial W_{0} \backslash \bigcup \delta_{k}=$ id. Since diam $\gamma_{k} \rightarrow 0$ and $\operatorname{diam} \delta_{k} \rightarrow$ $0, h$ is continuous. It is also injective by definition, so it is a homeomorphism onto its image. Notice that $h\left(\partial W_{0}\right) \supset \partial W$ by construction. On the other hand, $\partial W$ can not be a proper subset of $h\left(\partial W_{0}\right)$ (otherwise it would not disconnect the plane). Thus $\partial W=h\left(\partial W_{0}\right)$ is a Jordan curve as was asserted.

Remark 9.1. Notice that the proof constructs an explicit homeomorphism between $\partial W^{0}$ and $\partial W$, which is close to the identity when $\sup \operatorname{diam}\left(W_{i}\right)$ is small, and which can be extended to a homeomorphism of $\bar{W}^{0}$ to $\bar{W}$ which is also close to the identity.

We will say that $F: \bigcup \Upsilon_{j} \rightarrow \Upsilon$ is normalized if $F(z)=F(0)+z^{2}+O\left(z^{3}\right)$ near 0 . 
Lemma 9.2. For every $\eta>0$, there exists $\kappa=\kappa(\eta)>0$ with the following property. Let $F$ be $\kappa$-good and normalized. There exists a homeomorphism $h: D_{4} \rightarrow \mathbb{C}$ which conjugates $P: D_{2} \rightarrow D_{4}$ to $F: h\left(D_{2}\right) \rightarrow h\left(D_{4}\right)$ such that

(1) $h$ is $\eta$ close to the identity,

(2) if $S$ is a non-central component of the domain of the first return map to $\Upsilon_{0}, S^{\prime}$ is a component of a preimage of $S$ by some iterate of $f$, and $Q$ is a Yoccoz puzzle piece for $P$ intersecting $h^{-1}\left(S^{\prime}\right)$, then $h^{-1}\left(S^{\prime}\right) \subset Q$.

Proof. Let $M^{0}$ be the domain bounded by the equator of $\Upsilon \backslash \Upsilon_{0}$, and $M^{k}=$ $f^{-k}\left(M^{0}\right)$. If $\kappa$ is big, then $\partial M^{0}$ is close to a circle centered at 0 , and $f \mid M^{1}$ is essentially quadratic. It follows that there exists $r$ and a quasiconformal map $\hat{h}: \bar{D}_{r} \backslash D_{r^{1 / 2}} \rightarrow \bar{M}^{0} \backslash M^{1}$ with dilatation close to 1 which conjugates $f \mid \partial M^{1}$ and $P \mid \partial D_{r^{1 / 2}}$ (notice that $r \geq \kappa$ and indeed $\ln r$ is close to $2 \bmod \left(M^{0} \backslash M^{1}\right)$ ). By the pullback argument, we obtain an extension $\hat{h}: \bar{D}_{r} \rightarrow \bar{M}^{0}$ with the same dilatation which is a hybrid conjugacy. By the normalization at the origin, $\hat{h} \mid D_{256}$ is uniformly close to the identity in the Euclidean metric, provided $\kappa$ is big.

Let $\mathcal{S}$ be the collection of non-central components of the domain of the first return map to $\Upsilon^{0}$, and let $\mathcal{S}^{\prime}$ be the collection of components of preimages of some $S \in \mathcal{S}$ by some iterate of $f$. Notice that if $S \in \mathcal{S}$, then there exists $\tilde{S} \subset \Upsilon^{0} \backslash \Upsilon^{1}$ such that the first return map $\phi: S \rightarrow \Upsilon^{0}$ extends to a univalent map $\phi: \tilde{S} \rightarrow \Upsilon$. If $S^{\prime} \in \mathcal{S}$ is such that $f^{k}\left(S^{\prime}\right)=S$, then $S^{\prime} \subset \Upsilon^{k} \backslash \Upsilon^{k+1}$ and the map $f^{k}: S^{\prime} \rightarrow S$ extends to a bigger domain $\tilde{S}^{\prime} \subset \Upsilon^{k} \backslash \Upsilon^{k+1}$ so that $f^{k}: \tilde{S}^{\prime} \rightarrow \tilde{S}$ is univalent. Notice that $\bmod \left(\tilde{S}^{\prime} \backslash S^{\prime}\right)=\bmod \left(\Upsilon \backslash \Upsilon^{0}\right) \geq \kappa$, so the hyperbolic diameter of $S^{\prime}$ in $\tilde{S}^{\prime}$ is very small. By the Schwarz Lemma, since $\tilde{S}^{\prime} \subset \Upsilon^{k} \backslash \Upsilon^{k+1} \subset M^{k} \backslash M^{k+2}$, the hyperbolic diameter of $S^{\prime}$ in $M^{k} \backslash \bar{M}^{k+2}$ is also very small.

Notice that if $S^{\prime} \in \mathcal{S}^{\prime}$ intersects $\partial \hat{h}\left(D_{4}\right)$, then $M^{k} \subset \mathbb{C} \backslash \overline{\hat{h}\left(D_{4}\right)}$ and $M^{k+2} \subset$ $\hat{h}\left(D_{4}\right)$, so $M^{k} \backslash M^{k+2} \subset \hat{h}\left(D_{256}\right) \backslash \hat{h}\left(D_{\sqrt{2}}\right)$, and the hyperbolic diameter of $S^{\prime}$ in $\hat{h}\left(D_{256}\right) \backslash \hat{h}\left(D_{\sqrt{2}}\right)$ is small. Let $B$ be the union of $\hat{h}\left(D_{4}\right)$ with all $S^{\prime} \subset \mathcal{S}^{\prime}$ which intersect $\hat{h}\left(D_{4}\right)$, and let $B^{0}$ be obtained from $\bar{B}$ by filling in the holes and taking the interior. By the previous lemma, $B^{0}$ is a Jordan domain. Moreover, $\partial B^{0}$ is close to $\partial \hat{h}\left(D_{4}\right)$ in the hyperbolic metric of $\hat{h}\left(D_{256}\right) \backslash \hat{h}\left(D_{\sqrt{2}}\right)$. Let $B^{k}=f^{-k}\left(B^{0}\right)$. Then (using Remark 9.1) there exists a homeomorphism $\tilde{h}: \bar{D}_{4} \backslash D_{2} \rightarrow \bar{B}^{0} \backslash B^{1}$ that conjugates $P \mid \partial D_{2}$ to $f \mid \partial B^{1}$, such that $\tilde{h} \circ \hat{h}^{-1}: \overline{\hat{h}\left(D_{4}\right)} \backslash \hat{h}\left(D_{2}\right) \rightarrow \bar{B}^{0} \backslash B^{1}$ is uniformly close to the identity in the hyperbolic metric of $\hat{h}\left(D_{256}\right) \backslash \hat{h}\left(D_{\sqrt{2}}\right)$.

The image by $\tilde{h}$ of the external rays $\gamma$ landing at the $\alpha$ fixed point of $P$ divide $\bar{B}^{0} \backslash B^{1}$ into finitely many (say, $k$ ) topological rectangles. Applying the previous lemma $k-1$ times, we obtain a new homeomorphism $h: \bar{D}_{4} \backslash D_{2} \rightarrow \bar{B}^{0} \backslash B^{1}$, such that $h\left(\gamma \cap \bar{D}_{4} \backslash D_{2}\right)$ is disjoint from $\bigcup_{S^{\prime} \in \mathcal{S}^{\prime}} S^{\prime}$ for all external rays $\gamma$. We may still require (see Remark 9.1) that $h \circ \hat{h}^{-1}: \overline{\hat{h}\left(D_{4}\right)} \backslash \hat{h}\left(D_{2}\right)$ is uniformly close to the identity in the hyperbolic metric of $\hat{h}\left(D_{256}\right) \backslash \hat{h}\left(D_{\sqrt{2}}\right)$.

Using the pullback argument, we can extend $h$ to a homeomorphism $h: \bar{D}_{4} \backslash$ $K(P) \rightarrow \bar{B}^{0} \backslash K(f)$. Since $\frac{P \mid D_{2}}{h} \backslash K(P), f \mid B^{1} \backslash K(f)$ are unbranched coverings, we conclude that $h \circ \hat{h}^{-1}: \overline{\hat{h}\left(D_{4}\right)} \backslash K(f) \rightarrow \bar{B}^{0} \backslash K(f)$ is uniformly close to the identity in the hyperbolic metric of $\hat{h}\left(D_{256}\right) \backslash K(f)$. In particular, $h \circ \hat{h}^{-1}$ extends through $K(f)$ as the identity, so $h$ extends to a homeomorphism $h: \bar{D}_{4} \rightarrow \bar{B}^{0}$ 
which is uniformly close to $\hat{h}$ in the hyperbolic metric of $\hat{h}\left(D_{256}\right)$. The map $h$ has the desired properties by construction.

We can use $h$ to transfer the principal nest $\hat{T}_{1} \supset \hat{T}_{2} \supset \ldots$ for $P$ to the principal nest $T_{1} \supset T_{2} \supset \ldots$ for $f, T_{k}=h\left(\hat{T}_{k}\right)$. Let us say that $F$ has $\left(N, \tau_{0}\right)$ bounded combinatorics if the first return time of $T_{\tau_{0}+1}$ to $T_{\tau_{0}}$ is bounded by $N$.

Lemma 9.3. For every $N>0, \tau_{0}>0$, there exists $\kappa>0, \xi>0$, with the following property. If $F$ is $\kappa$-good and has $\left(N, \tau_{0}\right)$ bounded combinatorics then

(1) $\left|T_{\tau_{0}}\right|>\xi$ if $F$ is normalized,

(2) the density of the domain of the first return map to $T_{\tau_{0}}$ is at most $1-\xi$.

Proof. Let us split the returning set of $T_{\tau_{0}}$ under the dynamics of $F$ into two parts: $X$ consists of points that return under the dynamics of $f$, and $Y$ is the complement.

Let us first show that $T_{\tau_{0}} \backslash X$ contains a disk of definite radius. Parameters $N$, $\tau_{0}$ specify a compact set of polynomials with the property that the domain of the first return map to $\hat{T}_{\tau_{0}}$ is not dense. In particular, there exists $\chi>0$ such that for any such polynomial, the non-returning set of $\hat{T}_{\tau_{0}}$ contains a disk of radius $\chi$. Taking $\eta<\chi / 10$ and $\kappa=\kappa(\eta)$ given by Lemma 9.2 , we conclude that $T_{\tau_{0}} \backslash X$ contains a disk of radius $\chi / 2$. In particular this implies the first statement, since $\left|T_{\tau_{0}}\right| \geq \chi^{2} / 4$.

Since the area of $T_{\tau_{0}}$ is bounded by 100 , the density of $X$ in $T_{\tau_{0}}$ is at most $1-\chi^{2} / 400$. If $x \in Y$, there exists $k>0$ minimal such that $f^{k}(x)$ belongs to some non-central component $S$ of the domain of the first return map to $\Upsilon^{0}$. The first return map $\phi: S \rightarrow \Upsilon^{0}$ extends to a univalent map $\phi: \tilde{S} \rightarrow \Upsilon$, where $\tilde{S} \subset \Upsilon^{0} \backslash \Upsilon^{1}$. Let $S^{\prime}, \tilde{S}^{\prime}$ be the components of $f^{-k}(S), f^{-k}(\tilde{S})$ containing $x$. Notice that $f^{k}: \tilde{S}^{\prime} \rightarrow \tilde{S}$ is univalent, so $f^{k}: S^{\prime} \rightarrow S$ has small distortion. It follows that the density of $Y \cap S^{\prime}$ in $S^{\prime}$ is at most $1-\sigma$ for some absolute $\sigma$. The domains $S^{\prime}(x)$ obtained in this way form a disjoint cover of $Y$ which does not intersect $X$. It follows that the density of $X \cup Y$ in $T_{\tau_{0}}$ is at most $1-\left(\sigma \chi^{2} / 400\right)$.

Let $\tau(F)=\tau(P)$ be the height of $F$.

Lemma 9.4 (see [L5]). There exists $\kappa>0, \rho>0$ such that if $F$ is $\kappa$-good, then $\bmod \left(T_{k} \backslash \bar{T}_{k+1}\right)>\rho k, k \leq \tau(F)+2$.

For the proof of the next lemma, we will need some combinatorial preparation for dealing with first return maps. Let us denote the first return map to $T_{k}$ by $R_{k}: \bigcup T_{k}^{j} \rightarrow T_{k}$, where $j$ takes values in $\mathbb{Z}$ and we set $T_{k+1}=T_{k}^{0}$. Let $\Omega$ be the set of finite sequences of non-zero integers $\underline{d}$. The length of $\underline{d}$ will be denoted $|\underline{d}|$ (we allow $|\underline{d}|=0)$. If $\underline{d}=\left(j_{1}, \ldots, j_{m}\right)$, let $T_{k}^{\underline{d}}=\left\{x \in T_{k}, R_{k}^{i-1}(x) \in T_{k}^{j_{i}}, 1 \leq i \leq m\right\}$, and let $R_{k}^{\frac{d}{k}}=R^{m} \mid T_{k}^{\frac{d}{k}}$, so that $R_{k}^{\frac{d}{k}}: T_{k}^{\frac{d}{k}} \rightarrow T_{k}$ is univalent. Let $W_{k}^{\frac{d}{k}}=\left(R_{k}^{\frac{d}{k}}\right)^{-1}\left(T_{k+1}\right)$. The map $L_{k}: \bigcup W_{k}^{d} \rightarrow T_{k+1}, L_{k} \mid W_{k}^{d}=R_{k}^{d}$ is the first landing map from $T_{k}$ to $T_{k+1}$, and its distortion is small provided $\bmod \left(T_{k} \backslash T_{k+1}\right)$ is big.

Lemma 9.5. There exists $\kappa>0, \tau>0, \lambda_{1}<1$ such that for $\tau \leq k \leq \tau(F)+2$ we have

(1) Let $Y_{k}$ be the union of $T_{k}^{j}$ such that the distortion of $R_{k} \mid T_{k}^{j}$ is at least $1+\lambda_{1}^{k}$. Then $\left|Y_{k}\right| \leq \lambda_{1}^{k}\left|T_{k}\right|$.

(2) Let $Z_{k+1}$ be the union of connected components of $\left(R_{k} \mid T_{k+1}\right)^{-1}\left(\bigcup T_{k}^{j}\right)$ where the distortion of $R_{k}^{2}$ is at least $1+\lambda_{1}^{k}$. Then $\left|Z_{k+1}\right| \leq \lambda_{1}^{k}\left|T_{k+1}\right|$. 
Proof. Let $R_{k} \mid T_{k}^{j}=F^{m}$. It is easy to see that $F^{m-1} \mid F\left(T_{k}^{j}\right)$ extends to a univalent map onto $T_{k-1}$. By the previous lemma, $F^{m-1} \mid F\left(T_{k}^{j}\right)$ has exponentially small distortion in $k, \tau \leq k \leq \tau(F)+2$.

By the previous lemma, if $\tau \leq k \leq \tau(F)$, the hyperbolic diameter of any $T_{k}^{j}$ in $T_{k}$ is exponentially small. Thus, the distortion of $F \mid T_{k}^{j}$ is also exponentially small in $k$, provided $T_{k}^{j}$ is not exponentially close to 0 in the hyperbolic metric of $T_{k}$. We conclude that $Y_{k}$ is contained in an exponentially small hyperbolic neighborhood of 0 in $T_{k}$. The first assertion follows.

Let $D$ be a component of $\left(R_{k} \mid T_{k+1}\right)^{-1}\left(\bigcup T_{k}^{j}\right)$. By the previous lemma, the hyperbolic diameter of $D$ in $T_{k+1}$ is exponentially small. If the distortion of $R_{k} \mid D$ is not exponentially small, then the distortion of either $R_{k} \mid\left(R_{k}(D)\right)$ or of $R_{k} \mid D$ is not exponentially small. In the first case, $D \subset\left(R_{k} \mid T_{k+1}\right)^{-1}\left(Y_{k}\right)$. In the second case, we conclude as before that $D$ is contained in an exponentially small hyperbolic neighborhood of 0 in $T_{k+1}$. Thus $Z_{k+1}$ is contained in the union of 3 sets with exponentially small hyperbolic diameter in $T_{k+1}$. The second assertion follows.

Lemma 9.6. There exists $\tau_{0}>0$ such that for every $N>0$, there exists $\kappa>0, \lambda<$ 1 with the following property. If $F$ is $\kappa$-good, has $\left(N, \tau_{0}\right)$ bounded combinatorics, and $\tau_{0} \leq k \leq \tau(F)+2$, then the density of the domain of the first return map to $T_{k}$ is at most $\lambda^{k}$.

Proof. In the proof, $\lambda$ 's will denote constants which are definitely smaller than one, depending only on $\rho$ from Lemma 9.4.

Let $Y_{k}, Z_{k}$, and $\lambda_{1}$ be as in the previous lemma. If $D \not \subset Z_{k+1}$ is a pullback of some $T_{k}^{j}$ under $R_{k} \mid T_{k+1}$, then the distortion of the map $R_{k}^{2}: D \rightarrow T_{k}$ is at most $1+\lambda_{1}^{k}$. Hence

$$
1-p\left(\bigcup T_{k+1}^{j} \mid D\right) \geq\left(1+\lambda_{1}^{k}\right)^{-1}\left(1-p\left(\bigcup W_{k}^{\underline{d}} \mid T_{k}\right)\right) .
$$

It follows that

$$
1-p\left(\bigcup T_{k+1}^{j} \mid T_{k+1}\right) \geq\left(1-p\left(Z_{k+1} \mid T_{k+1}\right)\right)\left(1+\lambda_{1}^{k}\right)^{-1}\left(1-p\left(\bigcup W_{k}^{\underline{d}} \mid T_{k}\right)\right) .
$$

By Lemma 9.5, $1-p\left(\bigcup T_{k+1}^{j} \mid T_{k+1}\right) \geq\left(1+\lambda_{1}^{k}\right)^{-1}\left(1-p\left(\bigcup T_{k}^{j} \mid T_{k}\right)\right)$. By Lemma 9.3, there exists $\xi>0$ such that $1-p\left(\bigcup T_{\tau_{0}}^{j} \mid T_{\tau_{0}}\right) \geq \xi$, so there exists $\sigma>0$ such that

$$
1-p\left(\bigcup T_{k}^{j} \mid T_{k}\right) \geq \sigma, \quad \tau_{0} \leq k \leq \tau(F)+2 .
$$

Notice that for $j \neq 0$ the map $R_{k}: T_{k}^{j} \rightarrow T_{k}$ carries the set $T_{k}^{j} \cap \bigcup W_{k} \frac{d}{k}$ to $\bigcup W_{k} \frac{d}{k}$. Hence, if $T_{k}^{j} \subset T_{k} \backslash Y_{k}$, then by the first part of Lemma 9.5, $p\left(\bigcup W_{k}^{\frac{d}{k}} \mid T_{k}^{j}\right) \leq$ $\left(1+\lambda_{1}^{k}\right) p\left(\bigcup W_{k} \frac{d}{k} \mid T_{k}\right)$. Hence

$$
p\left(\bigcup W_{k}^{\underline{d}} \mid T_{k}\right) \leq p\left(Y_{k} \mid T_{k}\right)+(1-\sigma)\left(1+\lambda_{1}^{k}\right) p\left(\bigcup W_{k}^{\underline{d}} \mid T_{k}\right),
$$

so if $k$ is big so that $(1-\sigma)\left(1+\lambda_{1}^{k}\right) \leq 1-(\sigma / 2),(9.2)$ and (9.3) imply

$$
p\left(\bigcup W_{k}^{\frac{d}{k}} \mid T_{k}\right) \leq \min \left\{1-\sigma, 2 \sigma^{-1} p\left(Y_{k} \mid T_{k}\right)\right\} .
$$

Hence $\left|\bigcup W_{k}^{\frac{d}{k}}\right| \leq \lambda_{2}^{k}\left|T_{k}\right|$. Putting this into (9.1), we get $\left|\bigcup T_{k}^{j}\right| \leq \lambda_{3}^{k}\left|T_{k}\right|$, as desired. 
Lemma 9.7. There exists $\tau_{0}>0$, such that for every $N>0$, there exists $\kappa>0, \tau>$ $\tau_{0}$, with the following property. If $F$ is $\kappa$-good, has $\left(N, \tau_{0}\right)$ bounded combinatorics and $\tau(F) \geq \tau$, then the first return map to $T_{\tau(F)}$ is $\kappa$-good.

Proof. Let $\kappa, \lambda$ be as in Lemma 9.6, let $\rho$ be as in Lemma 9.4, and let $\tau$ satisfy $\lambda^{\tau}<\kappa^{-1}, \tau \rho \geq \kappa$.

Theorem 9.8 (Lean case exists). There exists $\tau_{0}>0$, such that for every $N>0$, there exists $\tau>\tau_{0}$ with the following property. Let $f$ be an infinitely renormalizable quadratic-like map such that all renormalizations of $f$ have $\left(N, \tau_{0}\right)$ bounded combinatorics and height at least $\tau$. Then the Julia set of $f$ has Lebesgue measure zero. Moreover, if $f$ is a Feigenbaum map, then $\operatorname{HD}(J(f))<2$.

Proof. Let $\tau_{0}, \mathrm{~N}, \kappa$, and $\tau$ be as in the previous lemma. It is enough to prove the result for quadratic maps. In this case, we can consider a quadratic-like restriction $f=F: \Upsilon^{0} \rightarrow \Upsilon$ which is $\kappa$-good. By the previous lemma, there exists a domain $\Upsilon_{(1)}=T_{\tau(F)}$ such that the first return map to $\Upsilon_{(1)}$ is $\kappa$-good. Repeating this procedure, we obtain a sequence of domains $\Upsilon_{(n)}$ around 0 such that the first return map $F_{n}$ to $\Upsilon_{(n)}$ is $\kappa$-good. We also let $\Upsilon_{(0)}=\Upsilon$. Let $\Upsilon_{(n)}^{0}$ be the central domain of the first return map to $\Upsilon_{(n)}$. Let $f_{n}=F_{n}: \Upsilon_{(n)}^{0} \rightarrow \Upsilon_{(n)}$.

Let $M_{(n)}^{0}$ be the domain bounded by the equator of $\Upsilon_{(n)} \backslash \Upsilon_{(n)}^{0}$. Let $M_{(n)}^{k}=$ $f_{n}^{-k}\left(M_{(n)}^{0}\right)$. Let $\mathbb{V}^{n}=M_{(n)}^{k_{n}}$ where $k_{n}$ is minimal with $\operatorname{diam}\left(M_{(n)}^{k_{n}}\right)<100 \operatorname{diam}\left(J^{n}\right)$, and let $\mathbb{U}^{n}=f_{n}^{-1}\left(\mathbb{V}^{n}\right)$. Then $\mathbb{U}^{n}$ and $\mathbb{V}^{n}$ have the properties (P1)-(P4) of Lemma 2.3 (see the proof of Lemma 9.2). Let $V^{n}=f_{n}^{-k_{n}-2}\left(\Upsilon_{(n)}^{0}\right), U^{n}=f_{n}^{-1}\left(V^{n}\right)$, and $A^{n}=V^{n} \backslash U^{n}$. Then $U^{n}$ and $V^{n}$ satisfy the conditions (C1)-(C3) and (G1)-(G2) of $\S 2.7$.

We claim that the density of the domain of the first return map to $V^{n}$ is bounded by some $\lambda<1$. The argument is similar to the proof of assertion (2) of Lemma 9.3. If $x \in A^{n}$ is such that $F_{n}^{k}(x) \in V^{n}$ for some $k$, then there exists $k \geq k_{n}+4$, and there exists $k_{n}+3 \leq r_{x} \leq k-1$ such that $F_{n}^{l}(x) \in \Upsilon_{(n)} \backslash \Upsilon_{(n)}^{0}, k_{n}+3 \leq l \leq r_{x}$ and $F_{n}^{r_{x}+1}(x)$ belongs to the domain of the first return map (under $F_{n}$ ) to $\Upsilon_{(n)}^{0}$. Moreover, there exists $\tilde{S}_{x} \ni x$ such that $F_{n}^{r_{x}+1} \mid \tilde{S}_{x}$ is a univalent map onto $\Upsilon_{(n)}$. Let $S_{x}=\left(F_{n}^{r_{x}+1} \mid \tilde{S}_{x}\right)^{-1}\left(\Upsilon_{(n)}^{0}\right)$. Then $S_{x} \subset V^{n} \backslash U^{n}$ and the $S_{x}$ form a disjoint cover of the domain of the first return map to $V^{n} \backslash U^{n}$. Since $F_{n}^{r_{x}+1}: S_{x} \rightarrow \Upsilon_{(n)}^{0}$ has bounded distortion, one sees that there exists a definite (at least $1-C \kappa^{-1}>0$ ) probability that a point in $A^{n}$ never returns to $V^{n}$. Since $\operatorname{area}\left(A^{n}\right) \asymp \operatorname{area}\left(V^{n}\right)$, this concludes the claim.

Let $\Delta_{n}$ be the set of points in $\mathbb{V}^{0}$ that visit $V^{n}$. Each branch of the first landing map $\phi_{n}: \Delta_{n} \rightarrow V^{n}$ has a univalent extension to $\mathbb{V}^{n}$ and thus has bounded distortion. Notice that if $x \in \Delta_{n+1}$, then $\phi_{n}(x)$ belongs to the domain of the first return map to $V^{n}$. This implies that $p\left(\Delta_{n+1} \mid \Delta_{n}\right)<\tilde{\lambda}<1$, so area $\left(\Delta_{n}\right)$ decreases exponentially fast. By [L1], almost every $x \in J(f)$ is such that $0 \in \omega(x)$, so $\operatorname{area}(J)=\operatorname{area}\left(\bigcap \Delta_{n}\right)=0$.

Assume now that $f$ is Feigenbaum. In the notation of $\S 4$, we have shown that $\xi_{n} \geq 1-C \kappa^{-1}$. The same argument applied to $F_{m}$ shows that $\xi_{m, n} \geq 1-C \kappa^{-1}$. By Lemma 4.1, $\eta_{m, n}$ is exponentially small in $n-m$. Fix some $\epsilon>0$ small. For $0<\rho<1$ small, let

$$
\omega_{\rho}^{[j]}(\delta)=\sup \omega_{m, n}^{[j]}(\delta), \quad \xi_{\rho}=\sup \xi_{m, n}, \quad \eta_{\rho}=\sup \eta_{m, n},
$$


where we take the supremums over pairs $m<n$ such that $\rho^{1+\epsilon}<\rho_{m, n}<\rho^{1-\epsilon}$ (for every $m$, there exists such an $n$ provided $\rho$ is sufficiently small). Notice that such pairs satisfy $-C^{-1} \ln \rho \leq n-m \leq-C \ln \rho$ (where $C$ depends on $f$ through the geometric and combinatorial bounds), so $\eta_{\rho}<\rho^{v}$ for some $v>0$. Moreover, $\xi_{\rho} \geq 1-C \kappa^{-1}$. Putting together (4.9) and (4.10), we get that for every $\delta$ close to 2 (depending on $\rho$ ), we have $\omega_{\rho}^{[j+1]}(\delta) \leq P_{\rho}\left(\omega_{\rho}^{[j]}(\delta)\right)$, where

$$
P_{\rho}(x)=C \frac{\eta_{\rho}}{\rho^{1+\epsilon}}+\left(1-C^{-1}\left(\eta_{\rho}+\xi_{\rho}\right)+C \eta_{\rho} \xi_{\rho} \rho^{-2 \epsilon}\right) x+C \xi_{\rho} \rho^{1-\epsilon} x^{2} .
$$

If $\epsilon>0$ is small, then for every $\rho$ sufficiently small $P_{\rho}$ has a positive fixed point $s$ that attracts 0 , and we have $\lim _{j \rightarrow \infty} \omega_{\rho}^{[j]}(\delta) \leq s, \delta \approx 2$. This implies that $\delta_{\text {cr }}(f)<2$, so $\operatorname{HD}(J(f))<2$ by Theorem C.

\section{VARIOUS REMARKS AND OPEN PROBLEMS}

In this section we will formulate some related results, possible further generalizations, and open problems. Some of these issues will be elaborated in forthcoming notes.

10.1. Outstanding Problems. The following problems still remain open:

Problem 1. Are there Feigenbaum Julia sets with positive area?

We are inclined to believe that the answer is affirmative.

Problem 2. Are there Feigenbaum Julia sets with zero area but Hausdorff dimension 2 ?

According to Theorem B, the affirmative answer to the first question (together with certain a priori bounds) implies the affirmative answer to the second.

Problem 3. What is the area and dimension of the Julia set of the classical Feigenbaum quadratic polynomial $F_{2}$ corresponding to the doubling renormalizations?

It is unlikely that Problem 3 can be resolved without computer assistance.

All results of this paper extend naturally (with the same proofs) to the setting of unicritical polynomial-like maps of some fixed degree $d \geq 2$ (specific constants may depend on the degree). Let $F_{d}: z \mapsto z^{d}+c_{d}$ (where $d \geq 2$ is an even integer) be the doubling Feigenbaum map of degree $d$, and let $J_{d}=J\left(F_{d}\right)$.

Problem 4. What is area $\left(J_{d}\right)$ for big $d$ ?

In the case area $\left(J_{d}\right)=0$, it would be interesting to find out whether $\operatorname{HD}\left(J_{d}\right)=2$ for big $d$. (According to [LS], $\operatorname{HD}_{\text {hyp }}\left(J_{d}\right) \rightarrow 2$ as $d \rightarrow \infty$.)

10.2. Hausdorff dimension in the parameter space. By our method one can produce a fair amount of Feigenbaum parameters with a small Julia set:

Theorem 10.1. The set of parameters $c \in M$ such that $P_{c}: z \mapsto z^{2}+c$ is a Feigenbaum map with $\mathrm{HD}(J(f))<2$ has Hausdorff dimension at least 1 .

To see this, it is enough to use the method of [L7] to obtain large sets of Feigenbaum maps of high combinatorial type.

10.3. Hyperbolic dimension and critical exponents. Our methods of proving that $\operatorname{HD}_{\text {hyp }}(J)=\mathrm{HD}(J)$ and $\delta_{\text {cr }}=\delta_{*}$ can be applied to a much broader class of rational maps: it will be elaborated in forthcoming notes. 
10.4. Absolutely continuous invariant measures. Construction of invariant geometric measures is probably the most important traditional problem of ergodic theory. Here is an answer in our situation:

Theorem 10.2. For any Feigenbaum map, there is a $\sigma$-finite measure $\lambda$ equivalent to the $\delta_{\mathrm{cr}}$-conformal measure $\mu$.

To construct this measure, one can use the method of $[\mathrm{KL}]$.

Problem 5. Can the invariant geometric measure of a Feigenbaum map be finite? (Of course, it may happen only in the conservative case.)

10.5. Hausdorff measure. Conformal measures appear as a useful substitution of Hausdorff measures $h_{\delta}$ since in the non-hyperbolic dynamical situations the latter usually vanish. The following statement (which easily follows from Lemma B.8 from Appendix B) confirms this principle:

Proposition 10.3. Let $\delta=\operatorname{HD}(J)$ for a Feigenbaum Julia set $J$ with periodic combinatorics. If area $(J)=0$, then $h_{\delta}(J)=0$.

10.6. Conservativity/dissipativity of towers. The Trichotomy nicely translates to measure-theoretic properties of towers $\bar{f}$ (see Appendix B):

- Lean case: the Lebesgue measure is dissipative, supported on $\mathbb{C} \backslash J(\bar{f})$, and almost all orbits escape to infinity under the tower dynamics.

- Balanced case: the Lebesgue measure is conservative under the tower dynamics, and supported on $\mathbb{C} \backslash J(\bar{f})$.

- Black hole case: the Lebesgue measure is dissipative, supported on $J(\bar{f})$, and almost all orbits are attracted to the postcritical set $\mathcal{O}(\bar{f})$.

10.7. Real Fibonacci maps and wild attractors. In this section, we will consider a class $\mathcal{A}$ of "real generalized polynomial-like maps"

$$
f: \bigcup_{j=0}^{s} I_{j} \rightarrow I, \quad s \geq 1
$$

where

(1) $I_{j}$ are disjoint closed intervals contained in a 0 -symmetric closed interval $I$, and moreover $I_{j} \subset \operatorname{int} I$.

(2) One of the $I_{j}$ (conventionally $\left.I_{0}\right)$ is a 0 -symmetric interval and $f:\left(I_{0}, \partial I_{0}\right)$ $\rightarrow(I, \partial I)$ is a unimodal map which can be written as $\phi\left(|x|^{l}\right)$ where $\phi$ is a diffeomorphism with a negative Schwarzian derivative onto a neighborhood of $I$, and $l>1$ is a real number (the criticality of $f$ ).

(3) If $j \neq 0$, then $f:\left(I_{j}, \partial I_{j}\right) \rightarrow(I, \partial I)$ extends to a diffeomorphism with negative Schwarzian derivative onto a neighborhood of $I$.

(4) The critical point is recurrent and $\omega(0)$ is a minimal set.

(5) All intervals $I_{j}$ intersect $\omega(0)$.

(6) $f$ is not renormalizable in the classical sense: there exist no 0 -symmetric intervals $T \subset I_{0}$ such that the first return map to $T$ is a unimodal map.

We shall consider such maps up to rescaling. The real Julia set is defined as $J^{r}(f)=$ $\bigcap f^{-n}(I)$. If it has positive length (三 one-dimensional Lebesgue measure), then almost all orbits are attracted to $\omega(0)$, which is called a wild attractor.

The principal nest is an infinite sequence of intervals defined inductively by $I^{0}=I, I^{1}=I_{0}$, and $I^{n+1}$ as the domain of the first return to $I^{n}$ containing 0 . Let 
$I_{j}^{n}$ be the connected components of the first return map to $I^{n}$ which intersect $\omega(0)$ (conventionally, we let $0 \in I_{0}^{n}$ so that $I_{0}^{n}=I^{n+1}$ ). We let $f_{n}: \bigcup I_{j}^{n} \rightarrow I^{n}$ be the first return map, and we call $f_{n+1}$ the generalized renormalization of $f_{n}$.

We say that $f$ has a bounded geometry if all the intervals $I_{n}^{j}$ and all the gaps in between are commensurable with $I^{n}$ (with constants independent of $j$ and $n$ ). In this case $0<\operatorname{HD}(\omega(0))<1$, and one is interested in $\operatorname{HD}\left(J^{r}(f)\right)$.

Maps of class $\mathcal{A}$ with bounded geometry are quite similar to Feigenbaum maps, and our methods apply to this setting as well. There is one (temporary?) advantage however: by [BKNS], we know that wild attractors do exist for some maps $f \in \mathcal{A}$, so that our results concerning Julia sets with positive measure become unconditional in this context.

Though we will concentrate on the real setting described above, maps of class $\mathcal{A}$ have natural complex counterparts: we refer the reader to [LMil] and [Bu], as well as to a more recent work by Smania $[\mathrm{Sm}]$. Our methods apply equally well in this setting (where the existence of Julia sets of positive Lebesgue measure is still an open problem).

Maps of class $\mathcal{A}$ with $1<l \leq 2$ cannot have bounded geometry (see [L4] for $l=2$ and $[\mathrm{Sh}]$ for a recent generalization).

We say that $f \in \mathcal{A}$ is a Fibonacci map if for every $n$ the domain of $f_{n}$ has two components, $I_{0}^{n}$ and $I_{1}^{n}$, and $f_{n} \mid I_{1}^{n}=f_{n-1}$ while $f_{n} \mid I_{0}^{n}=f_{n-1}^{2}$. For $l=2$, geometry of Fibonacci maps was analyzed in [LMil]. A Fibonacci map with criticality $l>2$ has bounded geometry (see $[\mathrm{KN}]$ ).

For $l>2$ but close to 2 , the intervals $I_{0}^{n}$ and $I_{1}^{n}$ are much smaller than $I^{n}[\mathrm{KN}]$, and in this case one can show that $J^{r}(f)$ is lean at the critical point. Our methods then imply:

Theorem 10.4. There exists an $\epsilon>0$ such that if $f$ is a Fibonacci map of degree $l \in(2,2+\epsilon)$, then $\operatorname{HD}\left(J^{r}(f)\right)<1$.

On the other hand, it is proved in [BKNS] that if $f$ is a Fibonacci map of large degree $l$, then the real Julia set $J^{r}(f)$ has positive length (so that $f$ has a wild attractor). Then our methods imply:

Theorem 10.5. If $f$ is a Fibonacci map of sufficiently large degree $l$, then

$$
\operatorname{HD}_{\text {hyp }}\left(J^{r}(f)\right)<1 .
$$

If $l$ is an even integer, one can prove more by methods of complex dynamics:

Theorem 10.6 (see $[\mathrm{Bu}]$ ). If $l>2$ is an even integer, then there exists a unique fixed point of the generalized renormalization with Fibonacci combinatorics. It attracts all other Fibonacci maps with the same criticality.

It is generally believed that the same result is true with arbitrary criticalities:

Conjecture 10.7. For every $l>2$ there exists a unique fixed point of the generalized renormalization with the Fibonacci combinatorics.

Martens's method [M2] can probably be applied to prove existence, but the uniqueness part appears to be wide open at this point.

Let us denote by $f_{l}$ the fixed point of the Fibonacci generalized renormalization if it exists and is unique. By compactness (real a priori bounds), $f_{l}$ depends continuously on $l$ (over l's for which it is defined). Thus if Conjecture 10.7 holds, we can see a "phase transition" in the family $\left\{f_{l}\right\}_{l \in(2, \infty)}$. 
Theorem 10.8. Assume that Conjecture 10.7 holds. Then all three cases of the Trichotomy occur for $f_{l}, 2<l<\infty$ :

(1) (Lean case) For an open set of l's containing $(2,2+\epsilon), \operatorname{HD}_{\text {hyp }}\left(J^{r}(f)\right)=$ $\mathrm{HD}\left(J^{r}(f)\right)<1$.

(2) (Balanced case) For a compact non-empty set of l's, $\operatorname{HD}_{\text {hyp }}\left(J^{r}(f)\right)=$ $\mathrm{HD}\left(J^{r}(f)\right)=1$ but the length of $J^{r}(f)$ is zero.

(3) (Black hole case) For an open set of l's containing $(K, \infty), \operatorname{HD}_{\mathrm{hyp}}\left(J^{r}(f)\right)<$ 1 but $J^{r}(f)$ has positive length.

Proof. By Theorems 10.4 and 10.5, the lean and black hole cases do occur for some sets of $l$ 's containing intervals $(2,2+\epsilon)$ and $(K, \infty)$, respectively. To see that they occur for open sets of parameters, notice that both are equivalent to a finite criteria (existence of $n$ such that $\eta_{n} / \xi_{n}$ is either small or large, with constants depending continuously on $l$; see Theorems 5.5 and 5.8$)$. Since $(2, \infty)$ is connected, the balanced case must also occur.

Problem 6. Is there a unique value $l_{0}$ for which the balanced case occurs?

It seems unlikely that the balanced case can ever happen for an integer $l .{ }^{17}$

\section{ApPEndix A. Nice fundamental DOMAins}

Let $f: \mathbb{U} \rightarrow \mathbb{V}$ be a Feigenbaum map, and let $f_{n}: \mathbb{U}^{n} \rightarrow \mathbb{V}^{n}$ be its prerenormalizations satisfying properties (P1)-(P4) of Lemma 2.3. In this section we will construct a sequence of simply connected domains $V^{n}$ satisfying properties (C1)-(C3) and (G1)-(G2) from $§ 2.7$. Our construction is reminiscent of Sinai's construction of Markov partitions [Si].

A.1. From admissible pairs to nice domains. Take some $n \in \mathbb{N}$, and let $\mathcal{F}^{n}$ be the family of inverse branches of $f^{k}, k \geq 0$, which are well defined on $\mathbb{V}^{n}$.

Let $X^{n} \subset \mathbb{V}^{n}$. For $g \in \mathcal{F}^{n}$ (an inverse branch of $f^{k}$ ), a set $g\left(X^{n}\right) \subset \mathbb{V}$ is called a copy of $X^{n}$ of depth $k \geq 0$. In particular, let us consider the set $\mathcal{C}^{n}=\{g(0)\}_{g \in \mathcal{F}^{n}}$ of all "copies" of 0 . There is a natural one-to-one correspondence between $\mathcal{C}^{n}$ and $\mathcal{F}^{n}$ so that we can use $\mathcal{C}^{n}$ to label all copies $X_{x}^{n}$ of $X^{n}, x \in \mathcal{C}^{n}$.

Lemma A.1. Let $X^{n} \subset \mathbb{V}^{n}$ be such that $f_{n}^{-1}\left(X^{n}\right) \subset X^{n}$. Then $\bigcup_{k} f^{-k}\left(X^{n}\right)$ is the union of all copies of $X^{n}$.

Proof. Let $z \in \bigcup_{k} f^{-k}\left(X^{n}\right)$, and let $k$ be the first landing moment of $z$ to $X^{n}$. Let $D_{k} \equiv \mathbb{V}^{n}$, and let us define $D_{k-j}$ for $1 \leq j \leq k$ inductively as the connected component of $f^{-1}\left(D_{k-j+1}\right)$ containing $f^{k-j}(z)$. Let us show that $f^{k}: D_{0} \rightarrow \mathbb{V}^{n}$ is univalent. If this is not the case, then $0 \in D_{k-j}$ for some $1 \leq j \leq k$. Take the minimal such $j$. Then it is easy to see that $j$ is the renormalization period on level $n$ and $D_{k-j}=\mathbb{U}^{n}$. Hence $f^{k-j}(z) \in f_{n}^{-1}\left(X^{n}\right) \subset X^{n}$, contradicting to the choice of $k$.

It follows that $\left(f^{k} \mid D_{0}\right)^{-1}\left(X^{n}\right)$ is a copy of $X^{n}$ containing $z$.

A pair $\left(Y^{n}, X^{n}\right)$ of nested sets $X^{n} \subset Y^{n} \subset \mathbb{V}^{n}$ is called admissible if every copy of $Y^{n}$ that intersects $X^{n}$ is contained in $Y^{n}$.

\footnotetext{
${ }^{17}$ For the same reason it is unlikely that there exists any example of a complex Feigenbaum/Fibonacci periodic point of renormalization which falls into the Balanced case, since there are only countably many parameters (combinatorics and degree) to play with.
} 
Lemma A.2. Let $\left(Y^{n}, X^{n}\right)$ be an admissible pair.

(1) Let $x, y \in \mathcal{C}^{n}$ be such that $\operatorname{depth}(x) \leq \operatorname{depth}(y)$. If $X_{x}^{n} \cap Y_{y}^{n} \neq \emptyset$, then $Y_{y}^{n} \subset Y_{x}^{n}$.

(2) Assume $X^{n}$ is a domain. Let $W$ be a domain which is the union of some copies of $X^{n}$. If $X_{x}^{n} \subset W$ is a copy of $X^{n}$ of smallest depth, then $W \subset Y_{x}^{n}$.

Proof. First assertion. Let $k=\operatorname{depth}(x)$. Then $f^{k}\left(X_{x}^{n}\right)=X^{n} \operatorname{intersects} f^{k}\left(Y_{y}^{n}\right)=$ $Y_{f^{k}(y)}^{n}$, so $f^{k}\left(Y_{y}^{n}\right) \subset Y^{n}$. It follows that $Y_{y}^{n} \subset Y_{x}^{n}$.

Second assertion. Notice that there is a sequence $x=x_{1}, x_{2}, \ldots$ of points of $\mathcal{C}^{n}$ such that $W$ is equal to the increasing union of domains $X_{x_{1}}^{n} \cup \cdots \cup X_{x_{m}}^{n}$. Thus, we can restrict ourselves to the case where $W$ is itself a finite union of copies of $X^{n}$.

Let us carry induction in the number of copies, $m$. The assertion is obvious for $m=1$. For the induction step, let us consider connected components $U_{l}$ of $\bigcup_{i=2}^{m} X_{x_{i}}^{n}$. By the induction hypothesis, $U_{l} \subset Y_{x_{j}}^{n}$ for some $j=j(l) \in[2, m]$. Since the union $U_{l} \cup X_{x}^{n}$ is connected, $U_{l} \cap X_{x}^{n} \neq \emptyset$; all the more, $Y_{x_{j}}^{n} \cap X_{x}^{n} \neq \emptyset$. By the first assertion, $Y_{x_{j}}^{n} \subset Y_{x}^{n}$. All the more, $U_{l} \subset Y_{x}^{n}$ and hence

$$
W=X_{x}^{n} \cup \bigcup_{l} U_{l} \subset Y_{x}^{n}
$$

Let us say that an open set $X \subset \mathbb{V}$ is nice if $f^{k}(\partial X) \cap X=\emptyset$ for all $k \geq 0$ (compare [M1]). Let us mention some nice properties of nice sets:

Lemma A.3. Let $f$ be a quadratic-like map. Then

(N1) For any open set $X \subset \mathbb{V}, \cup f^{-k}(X)$ is nice.

(N2) Components of a nice set are nice.

(N3) If $X$ is a nice domain, then the filling of $X$ is nice, provided $f$ has no attracting periodic orbits.

(N4) Intersection of two nice sets is nice.

(N5) Preimages of nice sets are nice.

Proof. We will show (N3), as all the other properties are obvious. Let $Z$ be the filling of $X$. Then $\partial Z \subset \partial X$. If $z \in \partial Z$ is such that $f^{k}(z) \in Z$, then $f^{k}(z) \in D$ where $D$ is a Jordan disk with $\partial D \subset X$. Since $X$ is nice and $\partial Z$ is connected, this implies that $f^{k}(\partial Z) \subset D$, and hence $f^{k}(Z) \subset D$ as well. Thus $f$ has an attracting periodic point in $D$, a contradiction.

Notice that property (C3) states precisely that $V^{n}$ should be nice. The next lemma shows how to use certain admissible pairs to construct nice domains.

Lemma A.4. Let $\left(Y^{n}, X^{n}\right)$ be an admissible pair, where $Y^{n}$ is a simply connected domain and $X^{n}$ is a domain containing 0 satisfying $f_{n}^{-1}\left(X^{n}\right) \subset X^{n}$. Then the filled connected component $S^{n}$ of $\bigcup f^{-k}\left(X^{n}\right)$ containing 0 is a nice simply connected domain contained in $Y^{n}$, and such that $f_{n}^{-1}\left(S^{n}\right) \subset S^{n}$.

Proof. Let $Z^{n}$ be the connected component of $\bigcup f^{-k}\left(X^{n}\right)$ containing $0 . Z^{n}$ is nice by (N1) and (N2). By Lemma A.1, $Z^{n}$ is the union of some copies $X_{x_{i}}^{n}$ of $X^{n}$, $x_{i} \in \mathcal{C}^{n}$. The smallest depth of these copies (that is, 0 ) is reached on $X^{n} \equiv X_{0}^{n}$ itself. By the second assertion of Lemma A.2, $Z^{n} \subset Y^{n}$. By (N3), $S^{n}$ is nice as well. Since $Y^{n}$ is simply connected, $S^{n} \subset Y^{n}$, and since $X^{n} \subset S^{n}, f_{n}^{-1}\left(S^{n}\right) \cap S^{n} \neq \emptyset$, the nice property implies that $f_{n}^{-1}\left(S^{n}\right) \subset S^{n}$. 
A.2. Separation between some Julia copies. Let $p_{n}$ be the renormalization period on level $n$, i.e., $f_{n}=f^{p_{n}}$. Let $J^{n}=J\left(f_{n}\right)$, and let $J_{i}^{n}=f^{i}\left(J^{n}\right), i=$ $0,1, \ldots, p_{n}-1$. Notice that the $J_{i}^{n}$ are copies of $J^{n}$. There are two cases to distinguish: whether the $J_{i}^{n}$ are all disjoint (the primitive case) or not (the satellite case).

To distinguish those cases, recall that $f_{n}$ has two fixed points, conventionally denoted $\alpha_{n}$ and $\beta_{n}$, such that $J^{n} \backslash\left\{\alpha_{n}\right\}$ is disconnected while $J^{n} \backslash\left\{\beta_{n}\right\}$ is connected. The satellite case happens if and only if $n>0$ and $f_{n-1}$ is immediately renormalizable, that is, $\alpha_{n-1}=\beta_{n}$. In this case, $J_{i}^{n} \cap J_{j}^{n} \neq \emptyset, 0 \leq i<j \leq p_{n}-1$, if and only if $j-i=0 \bmod \frac{p_{n}}{p_{n-1}}$, and in this case $J_{i}^{n} \cap J_{j}^{n}=f^{i}\left(\beta_{n}\right)$.

A $(\nu \operatorname{diam} J)$-neighborhood of a set $J$ will be called the $\nu$-enlargement of $J$.

Lemma A.5. There exists $\nu>0$ such that if $J^{n} \cap J_{i}^{n}=\emptyset$, then the $\nu$-enlargement of $J^{n}$ is disjoint from $J_{i}^{n}$.

Proof. Let $m \leq n$ be minimal such that $J_{i}^{n} \subset J_{k}^{m}$ with $J_{k}^{m} \cap J^{m}=\emptyset$. Then $J_{k}^{m} \subset J^{m-1}$ (in the primitive case) or $J_{k}^{m} \subset J^{m-2}$ (in the satellite case). By a simple compactness argument, $J_{k}^{m}$ is disjoint from the $\nu$-enlargement of $J^{m}$. All the more, the smaller set $J_{i}^{n} \subset J_{k}^{m}$ is disjoint from the $\nu$-enlargement of $J^{n} \subset J^{m}$.

A.3. Construction of admissible pairs. Let $\nu$ be as in Lemma A.5, and fix $\rho \leq \nu$. Let $l \geq 0$ be minimal such that $\Gamma^{n} \equiv f_{n}^{-l}\left(\mathbb{U}^{n}\right)$ is contained in the intersection of the $\rho$-enlargement of $J^{n}$ with $\mathbb{U}^{n-1}$. Notice that:

(1) $\bmod \left(\Gamma^{n} \backslash f_{n}^{-1}\left(\bar{\Gamma}^{n}\right)\right)$ is definite (once $\rho$ is fixed).

(2) If $J_{i}^{n} \cap \Gamma^{n} \neq \emptyset$, then either $J_{i}^{n}=J^{n}$ or $f_{n-1}$ is immediately renormalizable and $J_{i}^{n}=f_{n-1}^{j}\left(J^{n}\right)$ for some $j$.

(3) If a copy $\Gamma_{x}^{n}$ of $\Gamma^{n}$ of depth $k>0$ intersects $J^{n}$, then $f_{n-1}$ is immediately renormalizable and $f^{k} \mid \Gamma_{x}^{n}$ is an iterate of $f_{n-1}$.

Indeed, $\Gamma_{x}^{n} \cap J^{n} \neq \emptyset$ implies that $f^{k}\left(\Gamma_{x}^{n}\right) \cap f^{k}\left(J^{n}\right)=\Gamma^{n} \cap f^{k}\left(J^{n}\right) \neq \emptyset$. By Lemma A.5, $J^{n}$ touches $f^{k}\left(J^{n}\right)$, so $f_{n-1}$ is immediately renormalizable, and $k=s p_{n-1}$ for some integer $s>0$. To conclude, we must show that $f^{j p_{n-1}}\left(\Gamma_{x}^{n}\right) \subset \mathbb{U}^{n-1}, 0 \leq j<s$. But if this is not the case, then the connected set $f^{j p_{n-1}}\left(\Gamma_{x}^{n}\right)$ intersects both $J^{n-1} \supset f^{j p_{n-1}}\left(J^{n}\right)$ and $\partial \mathbb{U}^{n-1}$. This implies that further iterates $f^{r p_{n-1}}\left(\Gamma_{x}^{n}\right), r>j$, have the same property, and in particular $\Gamma^{n}=f^{k}\left(\Gamma_{x}^{n}\right)$ intersects $\partial \mathbb{U}^{n-1}$, contradiction.

We will consider separately the primitive and satellite cases.

A.3.1. Primitive case. Assume that $f_{n-1}$ is not immediately renormalizable. In this case, any copy of $\Gamma^{n}$ of depth $k>0$ is disjoint from $J^{n}$.

Let $Y^{n}=f_{n}^{-1}\left(\Gamma^{n}\right)$. Notice that $\partial Y^{n}$ has bounded hyperbolic diameter in the hyperbolic metric of $\mathbb{V}^{n} \backslash J^{n}$.

If $Y_{x}^{n}$ is a copy of $Y^{n}$ of positive depth, then the hyperbolic diameter of $Y_{x}^{n}$ in $\mathbb{V} \backslash J^{n} \supset \Gamma_{x}^{n}$ is bounded (since $\bmod \left(\Gamma_{x}^{n} \backslash \bar{Y}_{x}^{n}\right)$ is definite). Notice that the hyperbolic metrics of $\mathbb{V} \backslash J^{n}$ and $\mathbb{V}^{n} \backslash J^{n}$ are comparable on $\mathbb{U}^{n} \supset Y^{n}$. Hence if $Y_{x}^{n} \cap \partial Y^{n} \neq \emptyset$, then the hyperbolic diameter of $Y_{x}^{n} \cap Y^{n}$ in the hyperbolic metric of $\mathbb{V}^{n} \backslash J^{n}$ is bounded by some $K$.

Let $s \geq 0$ be minimal such that the hyperbolic distance (in the hyperbolic metric of $\left.\mathbb{V}^{n} \backslash J^{n}\right)$ from any $z \in X^{n} \equiv f_{n}^{-s}\left(Y^{n}\right)$ to $\partial Y^{n}$ is greater than $K$. Then $\left(Y^{n}, X^{n}\right)$ is admissible. 
Notice also that the hyperbolic diameter of $\bar{Y}^{n} \backslash X^{n}$ (in the hyperbolic metric of $\mathbb{V}^{n} \backslash J^{n}$ ) is uniformly bounded.

A.3.2. Satellite case. Assume that $f_{n-1}$ is immediately renormalizable.

Lemma A.6. There exists a simply connected domain $Y^{n} \subset \Gamma^{n}$ such that

(1) $Y^{n}$ is nice for $f_{n-1}$ and $f_{n}^{-1}\left(Y^{n}\right) \subset Y^{n}$.

(2) There exists a finite set $F$ of copies of $\beta_{n}$ such that $Y^{n}$ is a neighborhood of $J^{n} \backslash F$.

(3) $\bmod \left(\Gamma^{n} \backslash \bar{Y}^{n}\right)$ is definite.

(4) The hyperbolic diameter of $\partial Y^{n}$ in $\mathbb{V}^{n} \backslash \mathcal{O}(f)$ is uniformly bounded.

(5) If $Y_{x}^{n} \cap \partial Y^{n} \neq \emptyset$, then $\Gamma_{x}^{n} \subset \Gamma^{n} \backslash J^{n}$.

(6) If $Y_{x}^{n} \cap \partial Y^{n} \neq \emptyset$, then the diameter of $Y_{x}^{n}$ in the hyperbolic metric of $\mathbb{V}^{n} \backslash J^{n}$ is bounded.

Proof. The properties described in Lemma 2.3 allow us to obtain a straightening of $f_{n-1}: \mathbb{U}^{n-1} \rightarrow \mathbb{V}^{n-1}$ with bounded dilatation, and thus to define equipotentials and external rays in $\mathbb{V}^{n-1}$. Let $E^{n-1}$ be the domain bounded by the equipotential of level 2. Let us cut $E^{n-1}$ by the external rays landing at $\alpha_{n-1}$, and let $Z^{n}$ be the connected component of 0 . Let us show that $Y^{n}=f_{n}^{-l}\left(Z^{n}\right)$ has all the desired properties if $l$ is large enough (independent of $n$ ).

Properties (1) and (2) are obvious. If $l$ is large enough, then $Y^{n} \subset \Gamma^{n}$. Moreover, then properties (3) and (4) are also valid for every fixed $l$.

Let us deal with property (5). Let us show first that if $Y_{x}^{n} \cap \partial Y^{n} \neq \emptyset$, then $\Gamma_{x}^{n} \cap J^{n}=\emptyset$. Indeed, if $\Gamma_{x}^{n} \cap J^{n} \neq \emptyset$, then $f^{k}: J_{x}^{n} \rightarrow J^{n}, k=\operatorname{depth}(x)$, is an iterate of $f_{n-1}$. But since $Y^{n}$ is nice for $f_{n-1}, Y_{x}^{n} \cap \partial Y^{n}=\emptyset$, a contradiction.

Let us now show that if $Y_{x}^{n} \cap \partial Y^{n} \neq \emptyset$, then $\Gamma_{x}^{n} \cap \partial \Gamma^{n}=\emptyset$. Notice that $\operatorname{diam}\left(Y_{x}^{n}\right) \asymp \operatorname{diam}\left(\Gamma_{x}^{n}\right), \bmod \left(\Gamma_{x}^{n} \backslash \bar{Y}_{x}^{n}\right)=\bmod \left(\Gamma^{n} \backslash \bar{Y}^{n}\right)$ is bounded from below (uniformly for all sufficiently big $l$ ), and $\partial Y^{n}$ is contained in an $\epsilon$-neighborhood of $J^{n}$ (where $\epsilon \rightarrow 0$ when $l \rightarrow \infty$ ). Those geometric properties together show that if $\Gamma_{x}^{n}$ intersects both boundaries, $\partial \Gamma^{n}$ and $\partial Y^{n}$ (so that $Y_{x}^{n}$ has size comparable with $\left.J^{n}\right)$, then $Y_{x}^{n} \cap \partial Y^{n} \neq \emptyset$ implies that $\Gamma_{x}^{n} \cap J^{n} \neq \emptyset$ for $l$ big enough, a contradiction. This proves (5).

The last property follows from the third, the fifth, and the fact that the hyperbolic metrics of $\mathbb{V} \backslash J^{n}$ and of $\mathbb{V}^{n} \backslash J^{n}$ are comparable on $\mathbb{U}^{n}$.

Let $X^{n}$ be the connected component of 0 of the interior of

$$
Y^{n} \backslash \bigcup_{Y_{x}^{n} \cap \partial Y^{n} \neq \emptyset} Y_{x}^{n} .
$$

Lemma A.7. The pair $\left(Y^{n}, X^{n}\right)$ is admissible. Moreover:

(1) $X^{n}$ is simply connected,

(2) $X^{n}$ is a neighborhood of $J^{n} \backslash F$,

(3) $f_{n}^{-1}\left(X^{n}\right) \subset X^{n}$,

(4) $\bar{Y}^{n} \backslash X^{n}$ has bounded hyperbolic diameter in $\mathbb{V}^{n} \backslash \mathcal{O}(f)$.

Proof. If $Y_{x}^{n} \cap X^{n} \neq \emptyset$, then $Y_{x}^{n} \cap \partial Y^{n} \neq \emptyset$ by definition of $X^{n}$, and hence $Y_{x}^{n} \subset Y^{n}$. Let us now check properties (1)-(4). 
(1) Notice that $X^{n}$ is a bounded connected component of the complement of the closure of the connected set

so it is simply connected.

$$
\bigcup_{\partial Y_{x}^{n} \cap \partial Y^{n} \neq \emptyset} \partial Y_{x}^{n}
$$

(2) By properties (2) and (6) of Lemma A.6, $X^{n}$ is a neighborhood of $J^{n} \backslash F$.

(3) If $Y_{x}^{n} \cap \partial Y^{n} \neq \emptyset$, then by property (5) of Lemma A.6, $\Gamma_{x}^{n} \subset \Gamma^{n} \backslash J^{n}$ which implies that $\operatorname{depth}(x) \geq p_{n}$ and in particular $f_{n}\left(Y_{x}^{n}\right)$ is a copy of $Y^{n}$. Thus, if $Y_{x}^{n} \cap f_{n}^{-1}\left(X^{n}\right) \neq \emptyset$ and $Y_{x}^{n} \cap \partial Y^{n} \neq \emptyset$, then there is a copy of $Y^{n}, f_{n}\left(Y_{x}^{n}\right)$, intersecting $X^{n}$ and $\partial\left(f_{n}\left(Y^{n}\right)\right)$, contradicting the definition of $X^{n}$. Since $J^{n} \backslash F \subset$ $X^{n}$ (by (2)), $f_{n}^{-1}\left(X^{n}\right)$ is a connected set intersecting $X^{n}$, and by the definition of $X^{n}$ it follows that $f_{n}^{-1}\left(X^{n}\right) \subset X^{n}$.

(4) By property (6), $\bar{Y}^{n} \backslash X^{n}$ has bounded hyperbolic diameter in $\mathbb{V}^{n} \backslash \mathcal{O}(f)$.

A.4. Construction of domains $U^{n}$ and $V^{n}$. Let $\left(Y^{n}, X^{n}\right), n \geq 0$, be given by the construction of the previous section. Let $S^{n}$ be given by Lemma A.4. We cannot set $V^{n}=S^{n}$ because this does not guarantee property (C2) (although it would possess the other properties).

This can be fixed as follows. Let $Q^{n}$ be the connected component of 0 of $\bigcap_{m<n} S^{m}$. By (N2) and (N4), $Q^{n}$ is nice. Let $V^{n}=f_{n}^{-1}\left(Q^{n}\right)$, and $U^{n}=f_{n}^{-1}\left(V^{n}\right)$. Then $V^{n}$ is nice by (N5), so $V^{n}$ has Property (C3). Notice that $J^{n} \backslash F \subset V^{n}$, where $F$ is a finite set of copies of $\beta_{n}$ (empty if $f_{n-1}$ is not immediately renormalizable). Since we also have $V^{n} \subset \mathbb{U}^{n}$, this implies (C1). To check (C2), assume there is $x \in V^{n} \cap \partial U^{n-1}$. Then $f_{n}(x) \in Q^{n} \subset Q^{n-1}$ and $f_{n-1}^{2}(x) \in \partial Q^{n-1}$. Notice that $f_{n}(x)=f_{n-1}^{k}(x)$ where $k=p_{n} / p_{n-1} \geq 2$. So $f_{n-1}^{k-2}\left(\partial Q^{n-1}\right) \cap Q^{n-1} \neq \emptyset$, contradicting that $Q^{n-1}$ is nice.

We have so far checked the combinatorial properties (C1)-(C3), and we will now verify the geometric properties (G1)-(G2).

We first make a couple of remarks on the geometry of $S^{n}$. We have that $\partial S^{n}$ has a bounded hyperbolic diameter in $\mathbb{V}^{n} \backslash \mathcal{O}(f)$. Since $\mathbb{V}^{n} \cap \mathcal{O}(f) \subset S^{n}$, this implies that the Euclidean distance from $\partial S^{n}$ to $\mathcal{O}(f) \cup \partial \mathbb{V}^{n}$ is comparable with $\operatorname{diam}\left(J^{n}\right) \asymp$ $\operatorname{diam}\left(\mathcal{O}(f) \cap \mathbb{V}^{n}\right) \asymp \operatorname{diam}\left(\mathbb{V}^{n}\right)$. In particular, $S^{n}$ contains a neighborhood of 0 of radius comparable with $\operatorname{diam}\left(J^{n}\right)$. On the other hand, $S^{n} \subset \mathbb{V}^{n}$ is contained in a disk of radius $\operatorname{diam}\left(\mathbb{V}^{n}\right) \asymp \operatorname{diam}\left(J^{n}\right)$. Since $\operatorname{diam}\left(J^{n}\right)$ decreases exponentially fast, we see that there exists $v \geq 0$ such that $S^{n} \subset S^{m}$ whenever $n \geq m+v$.

Lemma A.8. The hyperbolic diameter of $\partial Q^{n}$ in $\mathbb{V}^{n} \backslash \mathcal{O}(f)$ is bounded.

Proof. For $m<n$, the Euclidean distance of points in $\partial S^{m}$ to $\mathcal{O}(f)$ is comparable with diam $J^{m} \geq \operatorname{diam}\left(J^{n}\right)$. This implies that the hyperbolic distance in $\mathbb{V}^{n} \backslash \mathcal{O}(f)$ of points in $\partial S^{m} \cap S^{n}$ to $\partial S^{n}$ is bounded. Since $\partial Q^{n} \subset \partial S^{n} \cup \bigcup_{m<n}\left(S^{n} \cap \partial S^{m}\right)$, the result follows.

Lemma A.9. The hyperbolic diameter of $\bar{V}^{n} \backslash U^{n}$ in $\mathbb{V}^{n} \backslash \mathcal{O}(f)$ is uniformly bounded.

Proof. Recall that if $g: X \rightarrow Y$ is a covering map of degree $n$ between hyperbolic Riemann surfaces and $Z \subset Y$ is such that $g^{-1}(Z)$ is connected, then the hyperbolic diameter of $g^{-1}(Z)$ in $X$ is at most $n$ times the hyperbolic diameter $d$ of $Z$ in $Y$.

Let us show first that $\partial V^{n}$ and $\partial U^{n}$ have bounded hyperbolic diameter in $\mathbb{V}^{n} \backslash$ $\mathcal{O}(f)$. By the Schwarz Lemma, the hyperbolic diameter of $\partial V^{n}$ in $\mathbb{V}^{n} \backslash \mathcal{O}(f)$ is 
bounded by its hyperbolic diameter in $f_{n}^{-1}\left(\mathbb{V}^{n} \backslash \mathcal{O}(f)\right)$. Since $\partial V^{n}=f_{n}^{-1}\left(\partial Q^{n}\right)$ is connected, its hyperbolic diameter in $f_{n}^{-1}\left(\mathbb{V}^{n} \backslash \mathcal{O}(f)\right)$ is bounded by two times the hyperbolic diameter of $\partial Q^{n}$ in $\mathbb{V}^{n} \backslash \mathcal{O}(f)$, which is bounded by Lemma A.8. Applying this argument to $\partial U^{n}=f_{n}^{-1}\left(\partial V^{n}\right)$, we see that $\partial U^{n}$ also has a bounded hyperbolic diameter in $\mathbb{V}^{n} \backslash \mathcal{O}(f)$.

This implies that the Euclidean distance of every $x \in \bar{V}^{n} \backslash U^{n}$ to $\partial \mathbb{V}^{n} \cup \mathcal{O}(f)$ is comparable with diam $J^{n}$. So the hyperbolic distance of every $x \in \bar{V}^{n} \backslash U^{n}$ to $\partial V^{n} \cup \partial U^{n}$ in $\mathbb{V}^{n} \backslash \mathcal{O}(f)$ is bounded. The result follows.

Property (G1) is given by Lemma A.9. Let us show that (C1)-(C3) and (G1) imply (G2).

Since $\partial U^{n}$ has bounded hyperbolic diameter in $\mathbb{V}^{n} \backslash \mathcal{O}(f), U^{n} \supset \mathbb{V}^{n} \cap \mathcal{O}(f)$, and $\operatorname{diam}\left(\mathbb{V}^{n}\right) \asymp \operatorname{diam}\left(\mathbb{V}^{n} \cap \mathcal{O}(f)\right)$, it follows that $U^{n}$ contains a disk $D$ centered around 0 of radius comparable with $\operatorname{diam}\left(V^{n}\right)$. Thus $\operatorname{area}\left(U^{n}\right) \asymp\left(\operatorname{diam}\left(U^{n}\right)\right)^{2} \asymp$ $\left(\operatorname{diam}\left(V^{n}\right)\right)^{2}$. We must show that $\operatorname{area}\left(V^{n} \backslash U^{n}\right) \asymp \operatorname{area}\left(V^{n}\right)$.

Lemma A.10. There exists $k>0$ (independent of $n$ ) and a round disk $W^{n} \subset$ $V^{n} \backslash f_{n}^{-k}\left(\mathbb{U}^{n}\right)$ with $\operatorname{diam}\left(W^{n}\right) \asymp \operatorname{diam}\left(J^{n}\right)$.

Proof. This follows easily by a compactness argument, but we will show how it can be deduced from the construction, since this gives more explicit estimates.

In the primitive case, the construction implies that $V^{n} \supset X^{n} \supset f_{n}^{-l}\left(\mathbb{U}^{n}\right)$ for some $l$. The annulus $f_{n}^{-l-1}\left(\mathbb{V}^{n} \backslash \overline{\mathbb{U}}^{n}\right)$ has a definite modulus, and encircles $J^{n}$, so it contains a round disk of diameter comparable with $\operatorname{diam}\left(J^{n}\right)$. We can take $W^{n}$ as this disk and $k=l+1$.

In the satellite case, notice that for every $\epsilon>0$, there exists $r>0$ such that $f_{n}^{-r}\left(\mathbb{U}^{n}\right) \backslash Y^{n}$ can be tiled into a bounded number $N$ (independent of $\epsilon$ ) of sets of Euclidean diameter less than $\epsilon \operatorname{diam}\left(J^{n}\right)$ (contained in $\epsilon \operatorname{diam}\left(J^{n}\right)$-neighborhoods of points where $\partial Y^{n}$ touches $\left.J^{n}\right)$. Notice that $\partial V^{n}$ is contained in a bounded neighborhood of $\partial Y^{n}$ in the hyperbolic metric of $\mathbb{V}^{n} \backslash J^{n}$. This implies that we can choose $l$ such that $f^{-l}\left(\mathbb{U}^{n}\right) \backslash V^{n}$ is also the union of $N$ sets with Euclidean diameter less than $\operatorname{diam}\left(J^{n}\right) /(100 N)$. Since the annulus $f_{n}^{-l-1}\left(\mathbb{V}^{n} \backslash \overline{\mathbb{U}}^{n}\right)$ has a definite modulus and encircles $J^{n}, f_{n}^{-l-1}\left(\mathbb{V}^{n} \backslash \overline{\mathbb{U}}^{n}\right) \cap V^{n}$ contains a round disk of diameter comparable with $\operatorname{diam}\left(J^{n}\right)$. We can take $W^{n}$ as this disk and $k=l+1$.

Taking $W$ and $k$ as in Lemma A.10, we have $W \subset \bigcup_{j<k} f_{n}^{-j}\left(V^{n} \backslash U^{n}\right)$, so $\operatorname{area}\left(V^{n}\right) \asymp \operatorname{area}(W) \leq C$ area $\left(V^{n} \backslash U^{n}\right)$. This concludes the proof of (G2).

Remark A.1. Notice that this construction gives $V^{n}$ with additional properties:

(A1) $U^{n}$ contains a round disk around 0 of radius comparable with $\operatorname{diam}\left(V^{n}\right)$,

(A2) $V^{n}$ is a neighborhood of $J^{n} \backslash F$ where $F$ is a finite (bounded) set of copies of $\beta_{n}$. Moreover, if $f_{n-1}$ is not immediately renormalizable, then $F=\emptyset$.

Moreover, in the construction we have the freedom to choose $\rho$ small so that $\Gamma^{n}$, and hence $V^{n} \subset Y^{n} \subset \Gamma^{n}$, is contained in the $\rho$-enlargement of $J^{n}$. This implies that if $J_{x}^{n}$ is a copy of $J^{n}$ of depth $k>0$ which intersects $V^{n} \subset Y^{n}$, then $\operatorname{diam}\left(J_{x}^{n}\right)$ is much smaller than $\operatorname{diam}\left(J^{n}\right)$ (this is easy in the primitive case, and follows from the bounded shape of $\Gamma_{x}^{n}$ and assertion (5) in Lemma A.6 in the satellite case). Under these circumstances, we obtain:

(A3) If $V_{x}^{n}$ is a univalent pullback of $V^{n}$ of depth $k>0$ contained in $V^{n}$, then $\operatorname{diam}\left(V_{x}^{n}\right)$ is much smaller than $\operatorname{diam}\left(V^{n}\right)\left(\operatorname{since} \operatorname{diam}\left(V_{x}^{n}\right) \asymp \operatorname{diam}\left(J_{x}^{n}\right)\right)$. 
(A4) If $V_{x}^{n}$ is a univalent pullback of $V^{n}$ of depth $k>0$ contained in $V^{n}$, then $\left|D f^{k}(x)\right|>2$ for every $x \in V_{x}^{n}$. This follows from the Koebe Distortion Lemma and (A3).

Remark A.2. If $f$ is a periodic point of renormalization, $f_{p}(x)=\lambda f\left(\lambda^{-1} x\right)$, we may assume that $\mathbb{V}^{n+p}=\lambda \mathbb{V}^{n}$. In this case we can modify the above construction to obtain $V^{n}$, satisfying $V^{n+p}=\lambda V^{n}$ as follows.

We can construct $Y^{n}$ satisfying $Y^{n+p}=\lambda Y^{n}$. In the primitive case, we can easily get that $X^{n+p}=\lambda X^{n}$, but in the immediately renormalizable case we will only get $X^{n+p} \subset \lambda X^{n}$. To fix this, in the immediately renormalizable case we modify the construction by taking the connected component of 0 in the interior of $\bigcap_{k} \lambda^{-k} X^{n+k p}$ as the new definition of $X^{n}$. Then $X^{n+p}=\lambda X^{n}$, but this only gives $S^{n+p} \supset \lambda S^{n}$. We take $\bigcup_{k} \lambda^{-k} S^{n+k p}$ as the new definition of $S^{n}$. Then $S^{n+p}=\lambda S^{n}$, but we only have $Q^{n+p}=\lambda Q^{n}$ for large $n$. We take $Q^{n}=\lambda^{-r} Q^{n+r p}$ for $r$ large. Then $V^{n}=f_{n}^{-1}\left(Q^{n}\right)$ satisfies $V^{n+p}=\lambda V^{n}$. All properties (C1)-(C3), (G1)-(G2) and (A1)-(A4) can easily be verified as before.

Remark A.3. The only parameters determining the constants in this construction are the unbranched a priori bounds and the combinatorics. Actually, one can see that a lower bound on the unbranched a priori bounds implies an upper bound on the period of any immediate renormalizations. As a consequence, the combinatorics are only used to get a lower bound on $\nu$ (the spacing between non-touching $J_{i}^{n}$ ).

\section{Appendix B. Conformal measures on towers}

B.1. Tower limits of conformal measures. In this section, let $f: \mathbb{U} \rightarrow \mathbb{V}$ be a Feigenbaum map with stationary combinatorics, and let $f_{n}: \mathbb{U}^{n} \rightarrow \mathbb{V}^{n}$ be its prerenormalizations with domains satisfying Lemma 2.3. Let $\mathbb{A}^{n}=\mathbb{V}^{n} \backslash \mathbb{U}^{n}$. Consider rescalings

$$
f_{m}^{(n)}=T_{n} \circ f_{n+m} \circ T_{n}^{-1}: \mathbb{U}_{(n)}^{m} \rightarrow \mathbb{V}_{(n)}^{m}, \quad n=0,1,2, \ldots, m=-n,-n+1, \ldots,
$$

that normalize the maps $f_{0}^{(n)}=T_{n} \circ f_{n} \circ T_{n}^{-1}$.

Let $\mu$ be a $\delta$-conformal measure on $J(f)$. To study its local geometry, let us push it forward by dilations $T_{n}, n \in \mathbb{N}$, and normalize it to be 1 on $\mathbb{V}_{(n)}^{0}, \mu_{n}=$ $\left(T_{n}\right)_{*}(\mu) / \kappa_{n}$.

Lemma B.1. The measures $\mu_{n}$ are uniformly bounded on compact subsets of $\mathbb{C}$.

Proof. Take some $m, k \in \mathbb{Z}_{+}$, and consider domains $\mathbb{V}^{m}$ and $\mathbb{U}^{m+k}$. We will show that

$$
\mu\left(\mathbb{V}^{m}\right) \leq C_{k} \mu\left(\mathbb{U}^{m+k}\right),
$$

which after rescalings implies the assertion. Moreover, taking $R^{m} f$, we see that it is enough to prove (B.1) for $m=0$ with the constant $C_{k}$ depending only on $\bmod (f)$.

Since the straightening of $f$ is $K$-qc where $K$ depends only on $k$ and $\bmod (f)$, there exists an $N$ depending only on $\bmod (f)$ such that $f^{N} \mathbb{U}^{k}=\mathbb{V}$. Moreover, $\left|D f^{N}(z)\right| \leq C$, where $C$ depends on the same data. By $\delta$-conformality of $\mu$, we conclude that $\mu(\mathbb{V}) \leq C^{\delta} \mu\left(\mathbb{U}^{k}\right)$.

Hence the family of measures $\mu_{m}$ is precompact in the weak* topology on compact subsets. Let $\bar{\mu}$ be a limit of these measures. This measure is Radon (i.e., it assigns finite mass to compact sets) and $\delta$-conformal for the whole tower $\bar{f}=\left\{f_{m}\right\}_{m \in \mathbb{Z}}$. 
B.2. Ergodicity. A set $X \subset \mathbb{C}$ is called invariant with respect to the tower $\bar{f}$ if $f_{m}\left(X \cap \mathbb{U}^{m}\right) \subset X$ for all $m \in Z$. A measure $\bar{\mu}$ is called ergodic with respect to the tower if there is no decomposition $\mathbb{C}=X \cup Y$ into two disjoint measurable sets of positive measure invariant with respect to the tower.

Theorem B.2. Let $\bar{\mu}$ be a conformal measure of the tower $\bar{f}$ which is fully supported on either the Julia set $J(\bar{f})$ or on its complement $\mathbb{C} \backslash J(\bar{f})$. Then $\bar{\mu}$ is ergodic.

Proof. If $\bar{\mu}$ is supported on the Julia set $J(\bar{f})$, then Theorem 2.9 implies that it is ergodic.

Assume that $\bar{\mu}$ is supported on $\mathbb{C} \backslash J(\bar{f})$. Assume that there is a decomposition $\mathbb{C} \backslash J(\bar{f})=X \cup Y$ into two invariant measurable sets with positive measure. Without loss of generality we can assume that the inequality

$$
\bar{\mu}\left(Y \cap \mathbb{A}^{m}\right) \geq \bar{\mu}\left(X \cap \mathbb{A}^{m}\right)
$$

holds for infinitely many levels $m \in-\mathbb{N}$.

Let us cut each $\mathbb{A}^{m}$ by a smooth arc $\gamma$ of zero $\bar{\mu}$-measure to obtain a (topological) rectangle $\Delta^{m}$. Then there exists a compact subset $S \subset X \cap \mathbb{U}^{0}$ of positive measure whose forward orbit does not intersect the cuts $\gamma_{m}$. It follows that for each $m \in-\mathbb{N}$, this set can be covered by disjoint preimages $\Pi_{i}^{m}$ of $\Delta^{m}$ under iterates of $f_{m}$.

By [Mc3, Prop. 6.9], diam $\Pi_{i}^{m} \rightarrow 0$ as $m \rightarrow-\infty$. Hence the unions $\Pi^{m} \equiv \bigcup_{i} \Pi_{i}^{m}$ shrink to $S$ as $m \rightarrow-\infty$. It follows that $\bar{\mu}\left(\Pi^{m} \backslash S\right) \rightarrow 0$. Hence there exists a rectangle $\Pi_{i(m)}^{m}$ such that $\operatorname{dens}\left(S \mid \Pi_{i(m)}^{m}\right) \rightarrow 1$ as $m \rightarrow-\infty$.

On the other hand, since the rectangles $\Pi_{i}^{m}$ are mapped onto the $\Delta^{m}$ with bounded distortion, $\operatorname{dens}\left(S \mid \Pi_{i}^{m}\right) \leq 1-\epsilon<1$ for all $m$ satisfying (B.2). This contradiction proves the result.

Corollary B.3. Given an exponent $\delta$, a tower can have at most one normalized $\delta$ conformal measure supported on the Julia set $J(\bar{f})$, and at most one such a measure supported on the complement $\mathbb{C} \backslash J(\bar{f})$.

Thus, any tower conformal measure $\bar{\mu}$ may have at most two ergodic components, $\bar{\mu} \mid J(\bar{f})$ and $\bar{\mu} \mid \mathbb{C} \backslash J(\bar{f})$.

B.3. Scaling. In what follows, $\rho \in(0,1)$ will stand for the scaling factor of the renormalization fixed point.

Lemma B.4 (Scaling covariance). Let $\bar{\mu}$ be a conformal measure for the tower $\bar{f}$ fully supported either on $J(\bar{f})$, or on $\mathbb{C} \backslash J(\bar{f})$. Then there exists a $\sigma=\sigma(\bar{\mu})>0$ such that $T_{*} \bar{\mu}=\rho^{\sigma} \bar{\mu}$, and for all $r>0, \bar{\mu}\left(\mathbb{D}_{r}\right) \asymp r^{\sigma}$.

Proof. Since the Feigenbaum dilation $T z \mapsto \rho z$ conjugates $f_{m}$ to $f_{m-1}$, the measure $T_{*} \bar{\mu}$ is also conformal with the same exponent. By Corollary B.3, $T_{*} \bar{\mu}=\kappa \bar{\mu}$ with some $\kappa>0$. Hence $\bar{\mu}\left(\mathbb{D}_{r}\right)=\kappa \bar{\mu}\left(\mathbb{D}_{\rho r}\right)$ for all $r>0$. Thus

$$
\bar{\mu}\left(\mathbb{D}_{\rho^{m}}\right)=\kappa^{-m} \bar{\mu}(\mathbb{D}), \quad m= \pm 1, \pm 2, \ldots,
$$

and the statement follows with $\sigma=-(\log \kappa / \log \rho)$.

If $\bar{\mu}$ is a tower conformal measure with two ergodic components, then let

$$
\begin{aligned}
\sigma_{-} & \equiv \sigma_{-}(\bar{\mu})=\max \{\sigma(\bar{\mu} \mid J(\bar{f})), \sigma(\bar{\mu} \mid \mathbb{C} \backslash J(\bar{f}))\} \\
\sigma_{+} & \equiv \sigma_{+}(\bar{\mu})=\min \{\sigma(\bar{\mu} \mid J(\bar{f})), \sigma(\bar{\mu} \mid \mathbb{C} \backslash J(\bar{f}))\} .
\end{aligned}
$$

In the case of one ergodic component we set $\sigma_{+}=\sigma_{-}=\sigma(\bar{\mu})$. 
Corollary B.5. For any tower $\delta$-conformal measure $\bar{\mu}, \bar{\mu}\left(\mathbb{D}_{r}\right) \asymp r^{\sigma_{+}}$for $r \leq 1$ and $\bar{\mu}\left(\mathbb{D}_{r}\right) \asymp r^{\sigma_{-}}$for $r \geq 1$, where the constants are independent of $\bar{\mu}$.

Let $\bar{\mu}_{+}$be the tower conformal measure on the Julia set (if it exists), and $\bar{\mu}_{-}$be such a measure on the complement (if exists). Both measures are normalized to be 1 on $\mathbb{V}$.

Corollary B.6. Let $\mu$ be a conformal measure of $f$. Then

$$
\sigma_{+} \leq \liminf _{r \rightarrow 0} \frac{\log \mu\left(\mathbb{D}_{r}\right)}{\log r} \leq \lim _{r \rightarrow 0} \sup \frac{\log \mu\left(\mathbb{D}_{r}\right)}{\log r} \leq \sigma_{-} .
$$

Proof. The limit set of the measures $\mu_{n}$ consists of convex combinations $t \bar{\mu}_{+}+(1-$ $t) \bar{\mu}_{-}$. For any such combination $\bar{\mu}$, we have

$$
\rho^{\sigma_{-}} \bar{\mu}\left(\mathbb{D}_{r}\right) \leq \bar{\mu}\left(\mathbb{D}_{\rho r}\right) \leq \rho^{\sigma_{+}} \bar{\mu}\left(\mathbb{D}_{r}\right)
$$

for any $r>0$. Hence

$$
\rho^{\sigma_{-}+\epsilon} \mu\left(\mathbb{D}_{r}\right) \leq \mu\left(\mathbb{D}_{\rho r}\right) \leq \rho^{\sigma_{+}-\epsilon} \bar{\mu}\left(\mathbb{D}_{r}\right),
$$

provided $r$ is sufficiently small (depending on $\epsilon>0$ ), which implies the assertion.

Lemma B.7. If a tower conformal measure $\bar{\mu}$ is not ergodic, then

$$
\sigma_{+}=\sigma(\bar{\mu} \mid J(\bar{f}))<\sigma(\bar{\mu} \mid \mathbb{C} \backslash J(\bar{f}))=\sigma_{-} .
$$

Proof. Let $X=\mathbb{C} \backslash J(\bar{f}), Y=J(\bar{f})$. Assume that

$$
\sigma_{+}=\sigma(\bar{\mu} \mid X) \leq \sigma(\bar{\mu} \mid Y)=\sigma_{-} .
$$

Then by Lemma B.4,

$$
\bar{\mu}\left(X \cap \mathbb{A}^{m}\right)=\bar{\mu}\left(X \cap \mathbb{A}^{0}\right) \rho^{m \sigma_{+}} \quad \text { and } \quad \bar{\mu}\left(Y \cap \mathbb{A}^{m}\right)=\bar{\mu}\left(Y \cap \mathbb{A}^{0}\right) \rho^{m \sigma_{-}} .
$$

Hence there exists a $c>0$ such that $\bar{\mu}\left(Y \cap \mathbb{A}^{m}\right) \geq c \bar{\mu}\left(X \cap \mathbb{A}^{m}\right)$ for all $m \in-\mathbb{N}$. This leads to a contradiction in the same way that (B.2) led to a contradiction in the proof of Theorem B.2.

Lemma B.8. Assume that area $(J(f))=0$. Let $\bar{\mu}$ be a tower $\delta$-conformal measure.

(i) If $\delta<2$, then $\sigma_{-}(\bar{\mu})<\delta$.

(ii) If $\delta=2$, then $\sigma_{-}(\bar{\mu}) \leq 2$. Moreover, $\sigma_{+}(\bar{\mu})<2$ unless $\bar{\mu}$ is the Lebesgue measure. ${ }^{18}$

Proof. Since area $(J(f))=0$, there exists a compact set $S \subset \mathbb{D} \backslash J(\bar{f})$ of positive Lebesgue measure.

Let $\Delta^{m} \subset \mathbb{A}^{m}$ be the topological rectangles obtained by cutting the annuli $\mathbb{A}^{m}$ by arcs of zero $\bar{\mu}$-measure (as in the proof of Theorem B.2). Take a point $z \in S$, and consider a sequence of moments $n_{m}=n_{m}(z) \rightarrow \infty$ such that $\zeta_{m} \equiv f^{n_{m}} z \in \Delta^{m}$, $m \leq 0$. Pulling this rectangle back by $f^{n_{m}}$, we obtain a rectangle $\Pi^{m}=\Pi^{m}(z)$ containing $z$.

(i) Let $\delta<2$. Assume $\sigma_{-} \geq \delta$. Then

$$
\bar{\mu}\left(\Delta^{m}\right) \geq c\left(\operatorname{diam} \Delta^{m}\right)^{\sigma_{-}} \geq c\left(\operatorname{diam} \Delta^{m}\right)^{\delta}, \quad m \in-\mathbb{N} .
$$

\footnotetext{
${ }^{18}$ Thus, if $\bar{\mu}$ is supported on $J(\bar{f})$, then $\sigma(\bar{\mu})<2$.
} 
Let $g_{m}=f^{n_{m}}: \Pi^{m} \rightarrow \Delta^{m}$. By the dynamical $\delta$-covariance and bounded distortion,

$$
\bar{\mu}\left(\Pi^{m}\right) \geq a \frac{\bar{\mu}\left(\Delta^{m}\right)}{\left|D g_{m}(z)\right|^{\delta}} \geq a c \frac{\left(\operatorname{diam} \Delta^{m}\right)^{\delta}}{\left|D g_{m}(z)\right|^{\delta}} \asymp\left(\operatorname{diam} \Pi^{m}\right)^{\delta} .
$$

Hence

$$
\bar{\mu}\left(\Pi^{m}\right) / \operatorname{area}\left(\Pi^{m}\right) \rightarrow \infty \text { as } m \rightarrow-\infty .
$$

But for a given $m$, different rectangles $\Pi^{m}(z), z \in S$, are disjoint and cover the whole set $S$. It follows that $\bar{\mu}(S)=\infty$, a contradiction.

(ii) Let $\delta=2$. Note that the Lebesgue measure is a 2-conformal measure supported on $\mathbb{C} \backslash J(\bar{f})$. By ergodicity (Theorem B.2), $\bar{\mu} \mid \mathbb{C} \backslash J(\bar{f})$ is proportional to it. Let $\bar{\nu}=\bar{\mu}+$ area. By Lemma B.7, $\sigma_{-}(\bar{\nu})=\sigma($ area $)=2$ and $\sigma_{+}(\bar{\nu})<2$ unless $\bar{\nu}$ (and hence $\bar{\mu}$ ) is Lebesgue.

Lemma B.9. Assume area $(J)=0$ but $\operatorname{HD}(J)=2$. Let $\mu$ be a 2 -conformal measure on $J$. Then

$$
\frac{\log \mu\left(\mathbb{D}_{r}\right)}{\log r} \rightarrow 2 \quad \text { as } \quad r \rightarrow 0
$$

Proof. By Corollary B.6 and Lemma B.8,

$$
\lim _{r \rightarrow 0} \sup \frac{\log \mu\left(\mathbb{D}_{r}\right)}{\log r} \leq 2 \text {. }
$$

Assume

$$
\lim _{r \rightarrow 0} \inf \frac{\log \mu\left(\mathbb{D}_{r}\right)}{\log r}<2,
$$

so that $\mu\left(\mathbb{D}_{r_{k}}\right) \geq r_{k}^{\sigma}$ for some $\sigma<2$ and a sequence $r_{k} \rightarrow 0$. Then

$$
\mu\left(\mathbb{V}^{n_{k}}\right) \geq\left(\operatorname{diam} \mathbb{V}^{n_{k}}\right)^{\sigma} \asymp \rho^{\sigma n_{k}}
$$

for some sequence of $n_{k} \rightarrow \infty$.

Let $X_{n}=\bigcup_{k=0}^{\infty} f^{-k} \mathbb{V}^{n}$. Let us consider the landing map $\Lambda_{n}: X_{n} \rightarrow \mathbb{V}^{n}$ to $\mathbb{V}^{n}$ and the associated Poincaré series $\Xi_{2}\left(\Lambda_{n}, z\right), z \in \mathbb{V}^{n}$. For $z \in \mathbb{V}^{n}$, we have

$$
\mu\left(\mathbb{V}^{n}\right) \Xi_{2}\left(\Lambda_{n}, z\right) \asymp \mu\left(X_{n}\right)=1,
$$

and hence

$$
\Xi_{2}\left(\Lambda_{n_{k}}, z\right) \leq \rho^{-\sigma n_{k}}
$$

But then

$$
\eta_{n_{k}} \equiv \operatorname{area}\left(X_{n_{k}}\right) \asymp \operatorname{area}\left(\mathbb{V}^{n_{k}}\right) \Xi_{2}\left(\Lambda_{n_{k}}, z\right) \leq \rho^{2 n_{k}} \rho^{-\sigma n_{k}},
$$

contradicting Theorem 5.2.

Let $\mu$ be a 2-conformal measure on the Julia set $J$. We say that the Julia set is measure-theoretic hairy at the critical point if

$$
\frac{\pi}{\mu\left(\mathbb{D}_{r}\right)} \mu \circ r \rightarrow \text { area } \quad \text { as } \quad r \rightarrow 0 .
$$

Here convergence is understood in the weak*-topology on compact sets.

Proposition B.10. If $\operatorname{HD}(J)=2$, then $J$ is measure-theoretic hairy. 
Proof. If $\operatorname{area}(J)>0$, then the assertion is obvious since the critical point is the density point of $J$. So, assume $\operatorname{area}(J)=0$. Then any limit measure $\bar{\mu}$ on the tower $\bar{f}$ is 2-covariant not only by the tower dynamics but by the $\rho$-scaling as well. Hence $\bar{\mu}\left(\mathbb{D}_{r}\right) \asymp r^{2}$. Spreading it around using distortion estimates, we see that $\bar{\mu}\left(\mathbb{D}_{r}(z)\right) \asymp r^{2}$ near any point $z \in \mathbb{C}$. Hence $\bar{\mu}$ is equivalent to the Lebesgue measure. But the only absolutely continuous 2-conformal measure is Lebesgue.

Let us consider a function $\phi: \mathbb{R}_{+} \rightarrow \mathbb{R}_{+}$such that $\phi(r) \rightarrow \infty$ as $r \rightarrow 0$. Let us say that it is slowly varying (near the origin) if for any $\kappa<1, \phi(\kappa r) / \phi(r) \rightarrow 1$ as $r \rightarrow 0$. Clearly, such a function has sub-polynomial growth:

$$
\frac{\log \phi(r)}{\log r} \rightarrow 0 \quad \text { as } \quad r \rightarrow 0 .
$$

Corollary B.11. Assume area $(J)=0$ but $\operatorname{HD}(J)=2$. Let $\mu$ be a 2-conformal measure on $J$. Then $\mu\left(\mathbb{D}_{r}\right)=r^{2} \phi(r)$, where $\phi$ is a slowly varying (growing to $\infty$ ) function.

Proof. Let us show that $\phi(r) \rightarrow \infty$. Otherwise there would be a sequence of levels $n_{k} \rightarrow \infty$ such that $\mu\left(\mathbb{V}^{n_{k}}\right) \leq C$ area $\left(\mathbb{V}^{n_{k}}\right)$. Hence for $z \in \mathbb{V}^{n_{k}}$,

$$
\mu\left(X_{n_{k}}\right) \asymp \mu\left(\mathbb{V}^{n_{k}}\right) \Xi_{2}(z) \leq C \text { area }\left(\mathbb{V}^{n_{k}}\right) \Xi_{2}(z) \asymp \operatorname{area}\left(X_{n_{k}}\right) .
$$

(Here $X_{n}$ is as in Lemma B.9.) But this is impossible since $\mu\left(X_{n_{k}}\right)=1$ while $\operatorname{area}\left(X_{n_{k}}\right) \rightarrow 0$ as $k \rightarrow \infty$.

Furthermore, by Proposition B.10,

$$
\frac{\mu\left(\mathbb{D}_{\kappa r}\right)}{\mu\left(\mathbb{D}_{r}\right)} \rightarrow \kappa^{2} \quad \text { as } \quad r \rightarrow 0
$$

which implies that $\phi$ is slowly varying.

In fact, we have

Proposition B.12. Under the above circumstances, $\phi(r) \asymp \log \frac{1}{r}$ for sufficiently small $r$.

Proof. As in the proof of Lemma B.9, we have

$$
\left.\Xi_{2}\left(\Lambda_{n}, z\right) \asymp \frac{1}{\mu\left(\mathbb{V}^{n}\right)} \asymp \frac{1}{\rho^{2 n} \phi\left(\rho^{n}\right.}\right)
$$

and

$$
\eta_{n} \equiv \operatorname{area}\left(X_{n}\right) \asymp \operatorname{area}\left(\mathbb{V}^{n}\right) \Xi_{2}\left(\Lambda_{n_{k}}, z\right) \asymp \frac{\rho^{2 n}}{\rho^{2 n} \phi\left(\rho^{n}\right)}=\frac{1}{\phi\left(\rho^{n}\right)} .
$$

Since by Theorem $5.2, \eta_{n} \asymp 1 / n$, we obtain the right asymptotics for $r=\rho^{n}$. Since $\phi$ is slowly varying, we can interpolate it to the intermediate scales.

\section{ACKNOWLEDGEMENT}

This work was partly supported by the the Guggenheim Foundation, Clay Foundation, NSF and NSERC. It was done during the authors' meetings at SUNY at Stony Brook, University of Toronto, and Institut Henri Poincaré. The authors thank all these Institutions and Foundations for their hospitality and support. The authors also thank one of the referees for his suggestions that led to the enrichment of $\S 2$ and $\S 3$. Final touches to this paper were done while the first author was a Clay Research Fellow. 


\section{REFERENCES}

[Ag] I. Agol. Tameness of hyperbolic 3-manifolds. Preprint math.GT/0405568 (2004).

[Ah] L. Ahlfors. Mobius transformations in several dimensions, University of Minnesota, 1981. MR725161 (84m:30028)

[ADU] J. Aaronson, M. Denker, M. Urbański. Ergodic theory for Markov fibered systems and parabolic rational maps. Trans. AMS 337 (1993), 495-548. MR1107025 (94g:58116)

[AL] A. Avila \& M. Lyubich. Examples of Feigenbaum Julia sets with small Hausdorff dimension. In "Dynamics on the Riemann Sphere. A Bodil Branner Festschrift", Ed. Poul G. Hjorth, Carsten Lunde Petersen, European Mathematical Society (EMS), Zürich, pp. 71-87, 2006.

[Ba] J. Barnes. Conservative exact rational maps of the sphere. J. Math. Anal. Appl., v. 230 (1999), no. 2, 350-374. MR1672223 (2000d:37048)

[B] C.J. Bishop. Minkowski dimension and the Poincaré exponent. Michigan Math. J. 43 (1996), no. 2, 231-246. MR1398152 (98h:30061)

[BJ] C.J. Bishop \& P.W. Jones. Hausdorff dimension and Kleinian groups. Acta Math. 179 (1997), no. 1, 1-39. MR1484767 (98k:22043)

[BL] A. Blokh \& M. Lyubich. Attractors of maps of the interval. In: "Dynamical Systems and Ergodic Theory", Banach Center Publ., v. 23 (1989), 427-442. MR1102738 (92c:58068)

[BM] A. Blokh \& M. Misiurewicz. Attractors for graph critical rational maps. Trans. Amer. Math. Soc., v. 354 (2002), no. 2, 3639-3661. MR1911515 (2003j:37064)

[Bo] R. Bowen. Hausdorff dimension of quasi-circles. Publ. Math. IHES 50 (1979), 11-26. MR556580 (81g:57023)

[BKNS] H. Bruin., G. Keller, T. Nowicki \& S. van Strien. Wild Cantor attractors exist. Ann. of Math. (2) 143 (1996), 97-130. MR1370759 (96m:58145)

[Bu] X. Buff. Fibonacci fixed points of renormalization. Erg. Th. \& Dynam. Syst., v.20 (2000), 1287-1317. MR1786715 (2001m:37086)

[BC] X. Buff \& A. Chéritat. Quadratic Julia sets with positive area. Preprint math/0605514 (2006).

[CG] D. Calegari \& D. Gabai. Shrinkwrapping and taming of hyperbolic 3-manifolds. J. Amer. Math. Soc. (2006), 385-446. MR2188131 (2006g:57030)

[CJY] L. Carleson, P.W. Jones \& J.-C. Yoccoz. Julia and John. Bol. Soc. Brasil. Mat., v. 25 (1994), 1-31. MR1274760 (95d:30040)

[DH] A. Douady and J.H. Hubbard. On the dynamics of polynomial-like maps. Ann. Sc. Éc. Norm. Sup., v. 18 (1985), 287-343. MR816367 (87f:58083)

[DU] M. Denker and M. Urbański. On Sullivan's conformal measures for rational maps of the Riemann sphere. Nonlinearity 4 (1991), 365-384. MR1107011 (92f:58097)

[EL1] A. Eremenko and M. Lyubich. Iterations of entire functions. Soviet Math Dokl. v. 30 (1984), 592-594. MR769199 (86c:30044)

[EL2] A. Eremenko and M. Lyubich. Examples of entire functions with pathological dynamics. J. London Math. Soc., v.36 (1987), 458-468. MR918638 (89e:30047)

[GY] D. Gaidashev, M. Yampolski. Cylinder renormalization of Siegel disks. Preprint math/0603008 (2006). To appear in Exp. Math.

[GS] J. Graczyk, S. Smirnov. Non-uniform hyperbolicity in complex dynamics I,II. Preprint (http://www.math.kth.se/ stas/papers/).

[J] Y. Jiang. Infinitely renormalizable quadratic polynomials. Trans. Amer. Math. Soc., v. 352 (2000), 5077-5091. MR1675198 (2001b:37071)

[K] J. Kahn. A priori bounds for some infinitely renormalizable quadratics: I. Bounded primitive combinatorics. Preprint IMS at Stony Brook, \# 2006/5.

[KL] V. Kaimanovich and M. Lyubich. Conformal and harmonic measures on laminations associated with rational maps. Memoires of the AMS, v. 173 (2005). MR2111096 (2006b:37087)

[Kar] B. Karpińska. Hausdorff dimension of the hairs without endpoints for $\lambda \exp (z)$. C.R. Acad. Sci. Paris, Ser. I, v. 328 (1999), 1039-1044. MR1696203 (2000e:37054)

[KN] G. Keller, T. Nowicki. Fibonacci maps re(al)visited. Erg. Th. \& Dyn. Syst., v. 15 (1995), 99-120. MR1314971 (95k:58098)

[LS] G. Levin, G. Swiatek. Hausdorff dimension of Julia sets of Feigenbaum polynomials with high criticality. Comm. Math. Phys. 258 (2005), 135-148. MR2166843 (2007b:37104) 
[L1] M. Lyubich. Typical behavior of trajectories of a rational mapping of the sphere. Dokl. Akad. Nauk SSSR, v. 268 (1982), 29-32. MR687919 (84f:30036)

[L2] M. Lyubich. Measurable dynamics of the exponential. Sibirsk. Mat. Zb., v. 28 (1987), 111-127. MR924986 (89d:58071)

[L3] M. Lyubich. On the Lebesgue measure of the Julia set of a quadratic polynomial. Preprint IMS at Stony Brook, \# 1991/10.

[L4] M. Lyubich. Combinatorics, geometry and attractors of quasi-quadratic maps. Ann. Math, v. 140 (1994), 347-404. Note on the geometry of generalized parabolic towers. arXiv:math.DS/0212382. MR1298717 (95j:58108)

[L5] M. Lyubich. Dynamics of quadratic polynomials, I-II. Acta Math., v. 178 (1997), 185-297. MR1459261 (98e:58145)

[L6] M. Lyubich. Dynamics of quadratic polynomials, III. Parapuzzle and SBR measures. Asterisque, v. 261 (2000). Colloque en l'honneur d'Adrien Douady. MR1755441 (2002e:37060)

[L7] M. Lyubich. How big is the set of infinitely renormalizable quadratics? Voronezh Winter Mathematical Schools, 131-143, Amer. Math. Soc. Transl. Ser. 2, 184. MR1729930 (2001c:37041)

[L8] M. Lyubich. Feigenbaum-Coullet-Tresser Universality and Milnor's Hairiness Conjecture. Ann. Math. v. 149 (1999), 319-420. MR1689333 (2000d:37051)

[LMil] M. Lyubich, J. Milnor. The Fibonacci unimodal map. Journal of AMS, v. 6 (1993), 425457. MR1182670 (93h:58080)

[LM] M. Lyubich \& Y. Minsky. Laminations in holomorphic dynamics. J. Differential Geom., v. 47 (1997), 17-94. MR1601430 (98k:58191)

[M1] M. Martens. Distortion results and invariant Cantor sets of unimodal maps. Erg. Th. \& Dyn. Syst., v. 14 (1994), 331-349. MR1279474 (96c:58108)

[M2] M. Martens. The periodic points of renormalization. Ann. Math., v. 147 (1998), 543-584. MR1637651 (99h:58057)

[Mc1] C. McMullen. Area and Hausdorff dimension of Julia sets of entire functions. Trans. Amer. Math. Soc., v. 300 (1987), 339-342. MR871679 (88a:30057)

[Mc2] C. McMullen. Complex dynamics and renormalization. Annals of Math. Studies, v. 142, Princeton University Press, 1994. MR1312365 (96b:58097)

[Mc3] C. McMullen. Renormalization and 3-manifolds which fiber over the circle. Annals of Math. Studies, v. 135, Princeton University Press, 1996. MR1401347 (97f:57022)

[Mc4] C. McMullen. Self-similarity of Siegel disks and Hausdorff dimension of Julia sets. Acta Math. 180 (1998), no. 2, 247-292. MR1638776 (99f:58172)

[Mc5] C. McMullen. Hausdorff dimension and conformal dynamics II: Geometrically finite rational maps. Comm. Math. Helv., v. 75 (2000), 535-593. MR1789177 (2001m:37089)

[Mat] P. Mattila. Geometry of sets and measures in Euclidean spaces. Cambridge University Press, 1995. MR1333890 (96h:28006)

[MS] W. de Melo and S. van Strien. One-dimensional dynamics. Springer, 1993. MR1239171 (95a:58035)

[MU] V. Mayer and M. Urbański. Fractal measures of meromorphic functions of finite order. Dyn. Syst. 22 (2007), no. 2, 169-178. MR2327991

[N] P.J. Nicholls. Ergodic theory of discrete groups. Cambridge University Press, 1989. MR1041575 (91i:58104)

[Pa] S.J. Patterson. The limit set of a Fuchsian group. Acta Math. 136 (1976), 241-273. MR0450547 (56:8841)

[Pe] C.L. Petersen.Local connectivity of some Julia sets containing a circle with an irrational rotation. Acta Math. 177 (1996), no. 2, 163-224. MR1440932 (98h:58164)

[P] E.A. Prado. Ergodicity of conformal measures for unimodal polynomials. Conform. Geom. Dyn. 2 (1998), 29-44. MR1613051 (99g:58106)

[Pz1] F. Przytycki. Lyapunov characteristic exponents are non-negative. Proc. AMS 119 (1993), 309-317. MR1186141 (93k:58193)

[Pz2] F. Przytycki. On measure and Hausdorff dimension of Julia sets of holomorphic ColletEckmann maps. International Conference on Dynamical Systems (Montevideo, 1995), 167-181, Pitman Res. Notes Math. Ser., 362, Longman, Harlow, 1996. MR1460803 (98i:58198) 
[PR] F. Przytycki \& S. Rohde. Porosity of Collet-Eckmann Julia sets. Fund. Math., v. 155 (1998), no. 2, 189-199. MR1606527 (2000b:37047)

[PU] F. Przytycki \& M. Urbański. Fractals in the plane - the ergodic theory methods. Cambridge Univ. Press, to appear.

[R] M. Rees. The exponential map is not recurrent. Math. Z. 191 (1986), 593-598. MR832817 (87g:58063)

[Sc] D. Schleicher. The structure of the Mandelbrot set. Preprint 1995.

[SZ] D. Schleicher \& J. Zimmer. Escaping points for exponential maps. J. London Math. Soc. 67 (2003), 380-400. MR1956142 (2003k:37067)

[Sh] W. Shen. Decay of geometry for unimodal maps: an elementary proof. Ann. of Math. 163 (2006), 383-404. MR2199221 (2007b:37078)

[Sh1] M. Shishikura. The Hausdorff dimension of the boundary of the Mandelbrot set and Julia sets. Ann. of Math. (2) 147 (1998), no. 2, 225-267. MR1626737 (2000f:37056)

[Sh2] M. Shishikura. Topological, geometric and complex analytic properties of Julia sets. Proceedings of the ICM-94, 886-895. Birkhäuser. MR1403988 (97e:58200)

[Si] Ya.G. Sinai. Markov partitions and $U$-diffeomorphisms. Funkional. Anal. i Prilozhen, v. 2 (1968), n. 1, 64-89. MR0233038 (38:1361)

[Sm] D. Smania. Puzzle geometry and rigidity: the Fibonacci cycle is hyperbolic. J. Amer. Math. Soc. 20 (2007), 629-673. MR2291915

[S1] D. Sullivan. The density at infinity of a discrete group of hyperbolic motions. Publ. Math. IHES 50 (1979), 419-450. MR556586 (81b:58031)

[S2] D. Sullivan. Growth of positive harmonic functions and Kleinian group limit sets of planar measure 0 and Hausdorff dimension 2. Lecture Notes Math., v. 894 (1981), 127144. MR655423 (83h:53054)

[S3] D. Sullivan. Conformal dynamical systems. Geometric dynamics (Rio de Janeiro, 1981), 725-752, Lecture Notes in Math., 1007, Springer, Berlin, 1983. MR730296 (85m:58112)

[S4] D. Sullivan. Entropy, Hausdorff measures new and old and limit sets of geometrically finite Kleinian groups. Acta Math. 153 (1984), 259-277. MR766265 (86c:58093)

[S5] D. Sullivan. Bounds, quadratic differentials, and renormalization conjectures. AMS Centennial Publications. 2: Mathematics into Twenty-first Century (1992). MR1184622 (93k:58194)

[Th] W. Thurston. The geometry and topology of 3-manifolds. Princeton University Lecture Notes, 1982.

[T] P. Tukia. The Hausdorff dimension of the limit set of a geometrically finite Kleinian group. Acta Math. 152 (1984), 127-140. MR736215 (85m:30031)

[U1] M. Urbański. Rational functions with no recurrent critical points. Ergodic Th. \& Dynam. Syst. 14 (1994), no. 2, 391-414. MR1279476 (95g:58191)

[U2] M. Urbański. Geometry and ergodic theory of conformal non-recurrent dynamics. Erg. Th. \& Dyn. Syst. 17 (1997), 1449-1476. MR1488329 (99j:58178)

[UZ] M. Urbański \& A. Zdunik. The finer geometry and dynamics of exponential family. Michigan Math. J. 51 (2003), 227-250. MR1992945 (2004d:37068)

[Y] B. Yarrington. Local connectivity and Lebesgue measure of polynomial Julia sets. Thesis, Stony Brook, 1995.

[Z] A. Zdunik. Parabolic orbifolds and the dimension of the maximal measure for rational maps. Invent. Math. 99 (1990), 627-649. MR1032883 (90m:58120)

CNRS UMR 7599, Laboratoire de Probabilités et Modèles aléatoires, Université Pierre et Marie Curie-Boîte courrier 188, 75252-Paris Cedex 05, France

E-mail address: artur@ccr.jussieu.fr

Department of Mathematics, University of Toronto, Ontario, Canada M5S 3G3

E-mail address: misha@math.toronto.edu

Current address: Mathematics Department and IMS, SUNY Stony Brook, Stony Brook, New York 11794

E-mail address: mlyubich@math.sunysb.edu 